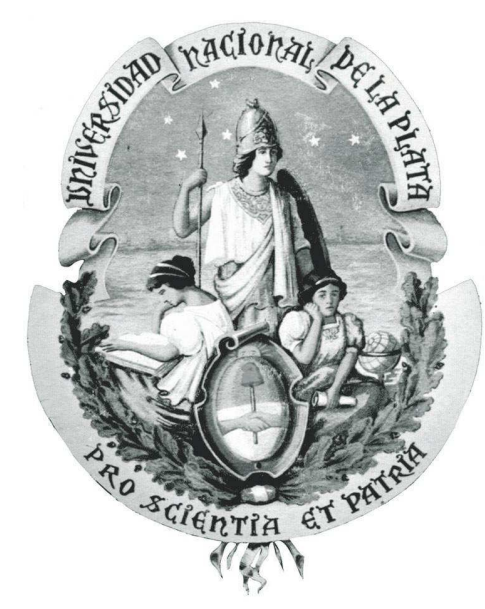

UNIVERSIDAD NACIONAL DE LA PLATA

Facultad de Ciencias Exactas

Departamento de Física

\title{
RELACIONES DE INCERTEZA Y ORIENTACIÓN DEL ESPÍN EN SISTEMAS ATÓMICOS REALISTAS
}

\author{
Tesis presentada para optar al título de Doctor de la Universidad de La Plata \\ en el área Ciencias Físicas
}

Lic. Diego A. Tielas

Director de tesis: Osvaldo Civitarese

Co-director de tesis: Marta Reboiro

Lugar de trabajo: Dto. de Física, Fac. Cs. Exactas, UNLP

La Plata, Marzo de 2011 
Tielas, Diego Alejandro

Relaciones de incerteza y orientación del espín en sistemas atómicos realistas. - 1a ed.

- La Plata : Universidad Nacional de La Plata, 2012.

E-Book.

ISBN 978-950-34-0808-7

1. Física Atómica. 2. Átomo. 3. Tesis de Doctorado. I. Título CDD 539.7

Fecha de catalogación: 22/11/2011 


\section{RELACIONES DE INCERTEZA Y ORIENTACIÓN DEL ESPÍN EN SISTEMAS ATÓMICOS REALISTAS}

\section{Resumen}

En esta tesis se analiza el problema de la persistencia en la orientación del espín y la reducción de las fluctuaciones en sus componentes, cuestión que se denotará genéricamente como "squeezing", en sistemas atómicos de dos y tres niveles activos. En particular, se estudian cadenas de $\mathrm{N}$ átomos de dos niveles en presencia de un campo de radiación. Se consideraron las interacciones entre átomos dependientes del sitio, la interacción de los átomos con el campo de radiación, y los efectos de disipación debidas a un ancho de frecuencia en el campo de radiación. También se aborda el problema de sistemas de átomos de tres niveles puestos en cavidades electromagnéticas de uno y dos modos, específicamente. Así mismo, se evalúa la dependencia del parámetro de squeezing al tener en cuenta interacciones del tipo dipolo-dipolo entre átomos.

Palabras clave: squeezing, disipación, interacciones dipolo-dipolo 


\section{Índice general}

Resumen

$\begin{array}{ll}\text { Introducción } & 2\end{array}$

1. Elementos teóricos y sistemas de interés $\quad 12$

1.1. Función de cuasi-distribución de Wigner . . . . . . . . . . . . . . . . . . . . 12

1.1.1. Medición de la función de Wigner y estados del campo electromagnético . . 14

1.2. Estados coherentes, representaciones y estados coherentes de espín . . . . . . . . 18

1.2.1. Estados coherentes . . . . . . . . . . . . . . . . . . . . . . . . . . 19

1.2.2. Representaciones de momento angular . . . . . . . . . . . . . . . 20

1.2.3. Esfera de Bloch . . . . . . . . . . . . . . . . . . . . . . . . 21

1.2.4. Estados coherentes de espín . . . . . . . . . . . . . . . . . 23

1.3. Relaciones de Incerteza y Squeezing . . . . . . . . . . . . . . . . . . . . 27

1.3.1. Squeezing en sistemas atómicos . . . . . . . . . . . . . . . . 30

1.3.2. Factor de squeezing . . . . . . . . . . . . . . . . . . 31

1.4. Modelo de Dicke y Jaynes-Cummings . . . . . . . . . . . . . . . . . . . . . 33

1.4.1. Hamiltoniano de Dicke para un átomo interactuando con radiación . . . . . 34

1.4.2. Modelo de Jaynes-Cummings . . . . . . . . . . . . . . . . . . . . . 38

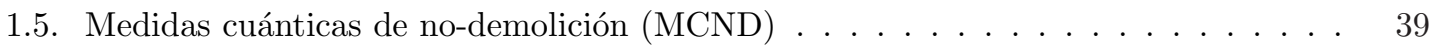

1.6. Sistemas de interés . . . . . . . . . . . . . . . . . . . . . . . . . . . . 42

1.6.1. Formulación de una MCND en sistemas de espines . . . . . . . . . . . . . . 42

1.6.2. Squeezing en trampas magneto ópticas . . . . . . . . . . . . . . . . . 43

1.6.3. Cavidades electromagnéticas . . . . . . . . . . . . . . 46

1.6.4. Redes ópticas . . . . . . . . . . . . . . . . . . . . . . . . . . . . . . 48

1.6.5. Trampas de iones . . . . . . . . . . . . . . . . . . . . . 49 
2. Squeezing en átomos de tres niveles $\quad 54$

2.1. Introducción . . . . . . . . . . . . . . . . . . . . . . 54

2.2. Formalismo . . . . . . . . . . . . . . . . . . 57

2.2.1. Solución exacta . . . . . . . . . . . . . . . 58

2.2.2. Evolución temporal . . . . . . . . . . . . . . . . . . . 58

2.2.3. Parámetro de squeezing en sistemas de tres niveles . . . . . . . . . . . . 59

2.2.4. Caso de un átomo . . . . . . . . . . . . . . . . . . . . 60

2.3. Resultados y discusión . . . . . . . . . . . . . . . . . . . . 62

2.4. Conclusiones del capítulo . . . . . . . . . . . . . . . . . . . . . . 64

3. Squeezing en átomos de tres niveles e interacciones dipolares $\quad 70$

3.1. Introdución . . . . . . . . . . . . . . . . . . . . . 70

3.2. Formalismo . . . . . . . . . . . . . . . . . . . 72

3.2.1. Solución exacta . . . . . . . . . . . . . . . . . 73

3.3. Resultados y discusión . . . . . . . . . . . . . . . . . . . . . . . . 74

3.4. Conclusiones del capítulo . . . . . . . . . . . . . . . . . . . . . . 78

4. Espín squeezing en presencia de disipación $\quad 84$

4.1. Introducción . . . . . . . . . . . . . . . . . . . . 84

4.2. Formalismo ........................ . . 86

4.2.1. Hamiltoniano . . . . . . . . . . . . . . . . . 87

4.2.2. Condiciones iniciales . . . . . . . . . . . . . . . . . . 88

4.2.3. Squeezing atómico . . . . . . . . . . . . . . . . . . 89

4.3. Resultados y discusión . . . . . . . . . . . . . . . . . . . . . . . 89

4.4. Conclusiones del capítulo . . . . . . . . . . . . . . . . . . . . . . 92

5. Squeezing en una cadena de espines con interacciones de largo alcance depen$\begin{array}{ll}\text { diente del sitio } & 98\end{array}$

5.1. Introducción . . . . . . . . . . . . . . . . . . . . . . 98

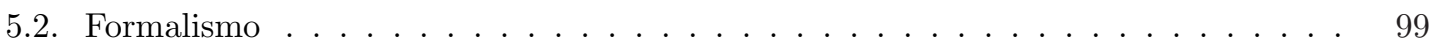

5.2.1. Cadenas de espines asimétricas . . . . . . . . . . . . . . . 100

5.2.2. Algunos casos analíticamente solubles . . . . . . . . . . . . . . . 102

5.3. Resultados y discusión . . . . . . . . . . . . . . . . . . . . . . 110

5.4. Conclusiones del capítulo . . . . . . . . . . . . . . . . . . . . . 113

$\begin{array}{lr}\text { Conclusiones } & 120\end{array}$ 
A. Cuantificación del campo electromagnético 124

B. Estados del campo electromagnético 128

B.1. Estados número . . . . . . . . . . . . . . . . . . . . . . . 128

B.2. Estados coherentes . . . . . . . . . . . . . . . . . . . 129

B.3. Estados comprimidos . . . . . . . . . . . . . . . . . . . 131

C. Átomos fríos $\quad 136$

C.1. Principios básicos del enfriamiento Doppler . . . . . . . . . . . . . . . . . . 136

C.2. Melaza óptica (Optical molasses) . . . . . . . . . . . . . . . . . . . . 138

C.3. Enfriamiento sub-Doppler . . . . . . . . . . . . . . . . . . . 140

C.4. Trampas magneto-ópticas . . . . . . . . . . . . . . . . . . . . . . 143

$\begin{array}{lr}\text { Agradecimientos } & 144\end{array}$

$\begin{array}{lr}\text { Bibliografía } & 146\end{array}$ 


\section{Introducción}

En Mecánica Cuántica las propiedades de un sistema físico están descriptas por operadores, y la medida de dichas propiedades corresponde a los valores esperados de los mismos sobre la función de estado que representa al sistema. Los valores esperados de los operadores dependen del estado del sistema. La precisión con la que se puede predecir el resultado de la medición depende de cuán bien definido está el estado respecto de la propiedad asociada al operador. A diferencia de la mecánica clásica, la mecánica cuántica pone límites a la precisión con la cual se puede conocer el estado de un sistema. El límite esta dado por las relaciones de incerteza de Heisenberg (Robertson, 1929), en las cuales el producto de incertezas de dos operadores es mayor o igual a cierta constante por el valor de su conmutador. Ejemplo de ello, son las relaciones de incerteza de los operadores posición y momento, o las relaciones de incertidumbre entre las diferentes componentes del momento angular.

Utilizando las relaciones de incertidumbre de Heisenberg, es posible clasificar los estados de un sistema respecto de los factores del producto de incertezas de dos operadores en dicho estado. Un estado que sature la desigualdad de Heisenberg, esto es, que alcance la igualdad de la expresión, se dirá que es un estado de mínima incerteza. Si además las incertezas de ambos operadores son iguales, estos definen el "límite cuántico estándar". En particular, un estado que disminuye las fluctuaciones de un operador, respecto a otro, por debajo del límite cuántico estándar se dirá que esta "comprimido", o que presenta "squeezing" ${ }^{1}$ en ese operador. Las primeras mediciones de estados que presentaban esta compresión fueron realizadas por Slusher et

\footnotetext{
${ }^{1}$ La traducción literal de la palabra inglesa squeezing es: exprimir, comprimir o estrujar. En esta tesis se la utilizará para denotar estados "comprimidos" en incerteza. Para una definición más precisa ver la Sección 1.3.
} 
al.(1985), en los laboratorios AT\&T. En este tipo de experimentos se generan estados del campo electromagnético donde la incerteza de una de sus cuadraturas ${ }^{2}$ toma valores por debajo del "ruido". Una de las aplicaciones inmediatas que se le dio a este tipo de estados del campo electromagnético fue para mejorar la sensibilidad de experimentos interferométricos (Hollenhorst and N., 1979; Lett et al., 1984) y actualmente se mantiene el interés en utilizarlos en detectores de ondas gravitatorias (Schanabel, 2008).

En la actualidad este tipo de estados tiene gran interés en el campo de investigación de la computación cuántica ya que, si en una variable se pudiera "imprimir, controlar y leer" información reduciendo su incerteza lo suficiente a expensas de que otras aumenten, se podría utilizar esa variable como un dispositivo de almacenamiento y procesamiento de información. Para un sistema cuántico aislado la unidad fundamental de información es el bit cuántico (o qubit), que básicamente es un sistema de dos niveles que puede ser preparado como superposición de dos estados. Los requerimientos óptimos para un hardware destinado a procesamiento de información cuántica (Di Vincenzo, 2000) pueden resumirse como:

1. El sistema cuántico (colección de qubits) debe poder inicializase en un estado bien definido.

2. Se debe poder realizar operaciones unitarias arbitrarias de manera que sea posible llevar el estado inicial a un estado entrelazado arbitrario.

3. La medida de los qubits debe realizarse con la mayor eficiencia posible.

Los dos primeros items demandan que los qubits estén lo mejor aislados posibles del entorno para asegurar estados iniciales puros y para preservar su carácter de superposición, además ellos deben poder interactuar fuertemente entre sí para poder volverse entrelazados. Dentro de las distintas propuestas de hardware cuántico la familia más atractiva de dispositivos de procesamiento de información cuántica provienen del área de la física atómica y óptica cuántica (Scully and Zubairy, 1997; Peng and Li, 1998; Klauder and Sudarshan, 2006), como lo son por ejemplo: las trampas de

\footnotetext{
${ }^{2}$ Operadores adimensionales que representan las componentes seno y coseno del campo electromagnético
} 
iones, las redes ópticas y las trampas magneto-ópticas. En estos dispositivos, átomos y fotones son manipulados en un medio controlado donde los acoplamientos son conocidos, ofreciendo un aislamiento del entorno que no se puede obtener en otros sistemas físicos. La idea general en este tipo de sistemas es utilizar átomos fríos, en un arreglo geométrico, como qubits que pueden servir de memorias o fuentes locales de entrelazamiento para procesamiento de la información. Esto es posible, dada la estabilidad de los estados que poseen los átomos fríos. Por otro lado, fotones individuales son la forma natural de comunicación cuántica ya que pueden atravesar largas distancias en la atmósfera, o fibras ópticas, con muy poca perturbación.

Entre estos dispositivos de computación cuántica con átomos fríos, los que utilizan átomos neutros (Brennen et al., 1999; Jaksch et al., 1999; Ahn et al., 2000), son particularmente interesantes, debido a los largos tiempos de coherencia de los estados atómicos internos y las bien desarrolladas técnicas de enfriamiento y captura en redes ópticas, trampas de luz fuera de resonancia, y micro trampas magnéticas (Briegel et al., 2000). En particular, los acoplamientos dipolo-dipolo entre estados de Rydberg proveen una interacción fuerte que es deseable para la implementación de compuertas de dos qubits en átomos neutros (con los tiempos típicos de operación de la compuerta mucho más pequeño que las escalas de tiempo asociadas con el movimiento de los átomos en el potencial confinante) (Jaksch et al., 2000).

En general, la interacción entre luz localizada y fuentes de espín, como lo son niveles atómicos, producen transferencia de espín (Lyakhov et al., 2007; Campos Venuti et al., 2007) y desviaciones de los valores esperados, en comparación con los valores dados por la conmutación estándar y las relaciones de incertidumbre (Walls and Zoller, 1981; Prakash and Kumar, 2005; Kitagawa and Ueda, 1993; Drummond and Ficek, 2004). Recientemente se han reportado notables resultados en la transferencia de espín entre estados atómicos y del campo electromagnético en experimentos de teleportación cuántica (Furusawa and Takei, 2007; Takei et al., 2005; Sherson et al., 2006; Yonezawa et al., 2007; Lyakhov et al., 2007; Brey et al., 2007; Rabl and Zoller, 2007; Campos Venuti et al., 2007; Romero-Isart et al., 2007; Joo et al., 2006; Fermani et al., 2007). 
En el estudio de fenómenos de transferencia de espín un factor adecuado para caracterizarlos es el llamado factor de squeezing que, en general, mide la compresión de la incerteza de alguna de las componentes de espín, respecto de otras, respetando el límite natural que pone las relaciones de incerteza. El estudio de las condiciones bajo las cuales se puede dar este fenómeno, de compresión en la incerteza, es motivo de una actividad creciente tanto experimental como teórica y particularmente lo es en el campo de la información cuántica, donde la transferencia de espín juega un papel decisivo en el procesamiento de la información.

Para los sistemas de espines existen varias definiciones del parámetro que da cuenta de la compresión en incerteza de un operador en dicho estado, o squeezing (Rojo, 2003). Una definición particularmente interesante es la dada por Kitagawa y Ueda (1993) ya que no solo da cuenta de la compresión de la incerteza de alguna de las componentes del espín, sino que además indica si los espines que componen al sistema se encuentran correlacionados. Recientemente Messikh (2003) mostró que para sistemas de dos átomos tipo Dicke la definición de squeezing dada por Kitagawa y Ueda (1993) es equivalente a la presencia de entrelazamiento. En general la presencia de squeezing en un sistema de átomos de dos niveles es una condición suficiente, aunque no necesaria, para que haya entrelazamiento (Sørensen and Mølmer, 1999). Lo que sigue es una revisión general de la literatura asociada al problema. Históricamente los estados comprimidos del campo electromagnético fueron los primeros en ser estudiados teórica y experimentalmente, y han recibido atención constante desde las primeras publicaciones hace más de 20 años (Walls, 1983; Hong and Mandel, 1985). Un repaso amplio del tema ha sido presentado en la referencia (Drummond and Ficek, 2004). Rangel et. al (2005) han obtenido expresiones analíticas para el operador densidad de un sistema de iones de dos niveles en un potencial armónico en una cavidad óptica, y han mostrado la transferencia de espín y el fenómeno de compresión en incerteza en el movimiento de los átomos. Estos autores han mostrado que se puede generar resurgimiento periódico de la compresión de incerteza de los estados de movimiento del ión y de los campos propios de la cavidad. La referencia (Rubin-Linares and Moya-Cessa, 2005) propone un esquema simple para 
medir squeezing y las propiedades de fase de un campo armónico en interacción con un sistema de átomos. En esta referencia se muestra que mediante la medida de la polarización atómica es posible medir propiedades de los campos de radiación. En general cuando se habla de la medición de las propiedades de un sector del sistema, sin incrementar la incerteza en una medición posterior, a expensas de la medición de las propiedades de otro sector, se habla de medidas cuánticas sin demolición (MCND). Un ejemplo de esto es medir el valor de una componente de espín asociada a un sistema atómico, midiendo las componentes de un campo de radicación, en interacción con los átomos, direcciones que no coinciden con la dirección del espín que se quiere medir (Takahashi et al., 1999). En la referencia (Pøulsen and Mølmer, 2001) se ha estudiado la transferencia de correlaciones de tipo cuántico desde el sector atómico del problema hacia el sector de radiación, por medio de la dispersión Raman de un pulso láser sobre una muestra de átomos previamente orientada. En esta referencia también se muestra que, bajo condiciones adecuadas, la información cuántica contenida en los estados colectivos atómicos, en este caso la maximización del espín en cierta dirección, puede ser transferida al pulso de luz. La compresión de incerteza del espín atómico bajo emisiones colectivas atómicas se estudió en la referencia (Yukalov and Yukalova, 2004), donde se desarrolló un método para gobernar la evolución temporal de la incerteza del espín atómico y se estudió la incidencia en las emisiones colectivas de los átomos por utilizar un estado comprimido del campo electromagnético como estado de vacío. La relación entre el entrelazamiento y propiedades del squeezing del espín para sistemas particulares, consistentes en átomos con dos niveles o bien cavidades con dos modos característicos, han sido estudiados en las referencias (Zeng et al., 2005; Josse et al., 2004). Wang (2001) estudió el squeezing del espín en un sistema no-lineal donde se enfatiza el carácter de estado coherente asociado al sector de espín de los estados atómicos involucrados. En la referencia (Wang and Sanders, 2003) se estudia la relación entre las cuadraturas del campo de radiación, y la compresión de incerteza de los estados atómicos. Genes et al. (2003) describen un conjunto de $N$ átomos de dos niveles interactuando con los modos de una cavidad, muestran que se puede obtener compresión en incerteza 
tanto para átomos como para los campos de radiación si el estado inicial de la cavidad guarda cierta relación de coherencia con respecto a las excitaciones atómicas. En particular se puede lograr compresión en incerteza si el estado inicial proveniente del campo de la cavidad tiene un número de coherencia entre estados que difiere en dos. En referencia a una solución analítica del problema se ha encontrado que la misma es válida si el número de átomos es mayor que el número medio de fotones considerados en la cavidad. En este límite el grado de compresión en incerteza del espín aumenta con el valor medio del número de fotones.

Un análisis detallado de un dispositivo lógico basado en interacciones dipolo-dipolo de átomos de Rydberg ópticamente atrapados se presentan en (Saffman and Walker, 2005; Saffman and Mølmer, 2008). Más recientemente en la referencia (Lukin et al., 2001), se prestó atención al fenómeno conocido como "bloqueo-dipolar". Cuando varios átomos están suficientemente cerca, la presencia de un átomo excitado causa un corrimiento en energía de todos los átomos que es lo suficientemente grande como para prevenir excitaciones resonantes de más de un átomo en la muestra (Lukin et al., 2001). Este fenómeno de "bloqueo-dipolar"tiene potencial para crear conjuntos fuertemente acoplados con un número moderado de átomos (Brion et al., 2007). Experimentos recientes han puesto de manifiesto señales de las interacciones de Rydberg necesarias para el "bloqueo-dipolar", en estados con número cuántico principal grande (Saffman and Mølmer, 2008; Walker and Saffman, 2008). En las referencias (Johnson et al., 2008; Saffman and Mølmer, 2009) se estudiaron las oscilaciones de Rabi entre estados fundamentales y de Rydberg en ${ }^{87} R b$. Ellos han observado oscilaciones coherentes de población para un átomo, mientras la presencia de dos o más átomos destruyen la coherencia de la oscilación. La generación de entrelazamiento multipartito vía bloqueo de Rydberg asimétrico se discute en (Saffman and Mølmer, 2009). Entre los sistemas en estudio, resultan de interés los conjuntos de átomos de tres niveles en presencia de campos láser e interactuando entre sí por medio de interacciones dipolo-dipolo. Por ejemplo se pueden ver en las referencias (MacOvei et al., 2005; Kiffner et al., 2007; Wang, 2009), donde se describe la implementación de la manipulación coherente de átomos de tres niveles interactuando vía interac- 
ciones dipolo-dipolo.

En particular, el problema de reconstrucción de espín en mediciones cuánticas de no-demolición (Shaffman et al., 2009; Teper et al., 2008; Oblak, 2005) está estrechamente relacionado con la compresión de la incerteza de espín (Hagelstein and Chaudhary, 2008; Nielsen and Mølmer, 2008; Echanis and et al., 2005; Korbicz et al., 2006).

Una característica común entre los modelos teóricos introducidos en las referencias que comentamos hasta el momento, es la consideración de la interacción entre niveles atómicos y fotones. Desde el punto de vista teórico, estos mecanismos de transferencia de espín entre componentes bosónicos (fotones) y los átomos, se pueden estudiar utilizando el Hamiltoniano de Dicke (1954) y también Hamiltonianos pertenecientes a la familia de los modelos llamados de Tavis-Cummings (Tavis and Cummings, 1968; Jaynes and Cummings, 1963; Buley and Cummings, 1964; Cummings, 1965). La estructura general de los Hamiltonianos que describen este tipo de interacciones pertenecen a la familia de acoplamientos presentados en el trabajo de revisión de Klein y Marshalek (1991). Estas formas son particularmente susceptibles de ser expresadas mediante expansiones o mapeos, bosónicos (Civitarese and Reboiro, 1998; Civitarese and Reboiro, 1999). Las técnicas de mapeo bosónico permiten la generalización, en formas simples de interacción que se han utilizado hasta ahora, como el de Hamiltoniano de Dicke (1954) o el de Tavis y Cummings (1963), por ejemplo. La extensión del esquema adoptado para átomos de dos niveles interactuando con un campo de radiación fue aplicado al estudio del squeezing en sistemas atómicos de tres niveles (Wadkiewicz et al., 1987; Ficek and Drummond, 1991a; Ficek and Drummond, 1991b; Javanainen and Gould, 1990). Recientemente en la referencia (Civitarese and Reboiro, 2010) se aplicaron representaciones algebraicas q-deformadas al tratamiento de sistemas de espines en interacción con un campo electromagnético demostrando la equivalencia entre el esquema de acoplamientos fermión-bosón. Para ello se utilizaron y extendieron técnicas algebraicas y computacionales que originariamente es común en el problema cuántico de muchos cuerpos y sistemas cuánticos con un número finito de grados de libertad. 
En esta tesis se estudia la aparición de squeezing de espín en sistemas espín-bosones, formados por niveles atómicos (espines) y campos de radiación (fotones) que son de interés en computación e información cuántica. En particular se estudiaron sistemas a temperatura cero, cuyos Hamiltonianos corresponden a sistemas realizables experimentalmente con átomos fríos. Estos son: átomos fríos en cavidades electromagnéticas, trampas magneto-ópticas, redes ópticas unidimensionales, y trampas de iones.

En el Capítulo 1 se presentan elementos teóricos que se utilizaron en esta tesis y, en forma breve, algunos experimentos con átomos fríos donde el fenómeno de squeezing es de interés. Se introduce la función de cuasi-distribución de Wigner, y como se la mide para el caso del campo electromagnético. Se presentan las representaciones de momento angular y los estados coherentes de espín. Además se comentan distintas definiciones del squeezing de espín, donde se presta especial atención a la definición introducida en el trabajo (Kitagawa and Ueda, 1993). Se introducen los modelos de Dike, Jaynes-Cummings y las medidas cuánticas de no-demolición (MCND). Para finalizar, se presentan en forma resumida algunos experimentos y propuestas experimentales donde el parámetro de squeezing es una cantidad de interés. Estos sistemas son: cavidades electromagnéticas, trampas magneto-ópticas, redes ópticas y trampas de iones.

En el Capítulo 2 se estudia la dependencia del squeezing en átomos de tres niveles, en una configuración escalera $(\Xi)$, que se encuentra en resonancia con un campo de radiación. Para esto, se introduce el modelo algebraico utilizado y se presentan los detalles para construir la evolución temporal de los operadores de espín de un Hamiltoniano que describe las excitaciones atómicas de $A$ átomos de tres niveles inducidas por el intercambio de fotones. Se estudia la dependencia del parámetro de squeezing respecto de las parametrizaciones de las constantes del modelo, el valor del número medio de fotones.

En el Capítulo 3 se extendió el modelo del Capítulo 2 para estudiar un sistema de átomos de tres niveles en una cavidad que posee dos modos (Dantan et al., 2003; Vogel and Blatt, 1992; Dalton et al., 1994; Ficek et al., 1995), en las configuraciones 
$\Xi, \Lambda$ y $V$. Para modelar las interacciones átomo-átomo se incluyeron interacciones efectivas dipolo-dipolo (Varada and Agarwal, 1992; Agarwal and Patnaik, 2001). Se estudió la aparición del squeezing atómico (Drummond and Ficek, 2004; Cerf et al., 2007) en base a la asimetría de las constante de acoplamiento en la interacción átomo-fotón del Hamiltoniano propuesto, así como también por la inclusión del término de interacción dipolo-dipolo (Civitarese and Reboiro, 2006; Reboiro, 2008). En el Capítulo 4 se estudia el squeezing de espín en una cadena de átomos localizados, de dos niveles, en interacción con fotones. El Hamiltoniano del sistema consiste de un término de radiación, un término de interacciones átomo-átomo y átomoradiación. Se modelan efectos disipativos a través de interacciones de intercambio átomo-fotón con constante de acoplamiento compleja.

En el Capítulo 5 se estudió numérica y analíticamente la dependencia del squeezing de espín para una cadena de átomos de dos niveles con interacciones periódicas dependientes del sitio. En particular se utilizó la aproximación TDA para estudiar la dependencia del squeezing con el número de átomos utilizados en la cadena.

Finalmente, se presentan las conclusiones generales obtenidas en el desarrollo de la tesis.

Se incluye un apéndice referido a la cuantización del campo electromagnético, squeezing del campo electromagnético y átomos fríos.

Los resultados obtenidos en el desarrollo de esta tesis fueron presentados en las siguientes reuniones científicas

- XL Latin American School of Physics (Elaf 2010): "Symmetries in Physics". Lugar: D.F., Mexico. Julio 2010.

Seminario: "Spin Squeezing in Atomic Systems"

- "II Quantum Information School and Workshop, Paraty 2009".

Lugar: Paraty, Rio de Janeiro, Brasil. Septiembre 2009.

Poster: "Spin Squeezing in a 1/2 spin chain with dissipative interactions"

- Giambiagi Winter School: "The Quantum Mechanics of the XXI Century: Ma- 
nipulation of Coherent Matter".

Lugar: UBA, Buenos Aires, Argentina. Julio 2009.

Poster:"Atomic Spin Squeezing in presence of dissipation".

Y han sido documentados en las siguientes publicaciones científicas internacionales con referato:

- Atomic Squeezing in three-level atoms with effective dipole-dipole atomic interactions.

O.Civitarese, M.Reboiro, L.Rebon and D.Tielas.

Phys. Lett. A 374 (21), p.2117-2121, (2010a).

- Squeezing in a spin-chain with site dependent periodic and long range interactions.

O.Civitarese, M.Reboiro, L.Rebon and D.Tielas.

Phys. Lett. A 374 (3), p.424-430, (2010b).

- Spin Squeezing in presence of dissipation.

O.Civitarese, M.Reboiro, L.Rebon and D.Tielas.

Phys. Lett. A 373 (7), p.754-758, (2009).

- Atomic spin squeezing in tree level atoms.

O.Civitarese, M.Reboiro, L.Rebon y D.Tielas.

Rev. Mex. Fis. 54 (3), p.24-29, (2008). 


\section{Capítulo 1}

\section{Elementos teóricos y sistemas de interés}

Este capítulo tiene por objetivo introducir algunos de los elementos teóricos que se utilizaron en el trabajo de tesis y presentar varios experimentos donde resulta de interés el estudio de la compresión en la incerteza del espín (spin squeezing). Se introduce la función (de cuasi-distribución) de Wigner y se muestran sus propiedades. Se describe el procedimiento experimental para medirla en el caso del campo electromagnético, y se explica como se clasifican los distintos estados en función de las incertezas de sus cuadraturas. A continuación se presentan las representaciones de espín que se utilizarán en la tesis y se construyen estados coherentes de espín. Seguidamente, se exponen distintas definiciones de squeezing de espín que se encuentran en la literatura y que utilizamos en capítulos posteriores. Para finalizar, se comenta la noción de media cuántica de no demolición (MCND), y se desarrollan varios experimentos y propuestas experimentales con átomos fríos donde la compresión en la incerteza de espín es de interés.

\subsection{Función de cuasi-distribución de Wigner}

El estado mecánico de una partícula clásica queda determinado al conocer su posición y su momento, lo cual puede ser representado como un punto en el espacio de fases. Si se tiene un conjunto de partículas, la probabilidad de encontrar una con un cierto valor en el espacio de fases está especificado por una distribución de 
probabilidades, esto es, la densidad de Liouville. Esta interpretación no se aplica al caso cuántico debido al carácter no-conmutativo de los operadores y al principio de incerteza. En mecánica cuántica la cuasi-distribución de Wigner juega un papel análogo, al de la distribución de Liouville. Esta cuasi-distribución no satisface todas las propiedades de una función de distribución, pero si cumple con todas las condiciones de borde que se requiere de una distribución. Por ejemplo, la distribución de Wigner toma valores negativos para estados que no tienen ninguna contraparte clásica, y es un indicador práctico de interferencias. La función de Wigner puede ser suavizada mediante un filtro de tamaño mayor a $\hbar$, por ejemplo, realizando una convolución Gaussiana en el espacio de las fases obteniendo así la representación de Husimi (Husimi, 1940; Leonhardt, 1997). Esto da lugar a una función semi-definida positiva la cual puede pensarse como una aproximación semi-clásica rudimentaria. La función de Wigner $P(x, p)$ se define como:

$$
P(x, p)=\frac{1}{\pi \hbar} \int_{-\infty}^{\infty} \psi^{*}(x+y) \psi(x-y) e^{2 i p y / \hbar} d y,
$$

donde $\psi$ es la función de onda y $x$ y $p$ son la posición y el momento, aunque podrían ser cualquier par de variables conjugadas. Se puede interpretar esta función de cuasidistribución como la transformada de Fourier de la densidad de probabilidad de que una partícula que "salta" del punto $x^{\prime}=x-y / 2$ al punto $x^{\prime \prime}=x+y / 2$. Esta función es simétrica en $x$ y $p$ ya que

$$
P(x, p)=\frac{1}{\pi \hbar} \int_{-\infty}^{\infty} \phi^{*}(p+q) \phi(p-q) e^{-2 i p y / \hbar} d q
$$

donde $\phi$ es la transformada de Fourier de $\psi$.

En el caso general, donde se incluyen estado mezcla, se define la transformación de Wigner sobre la matriz densidad del sistema como

$$
P(x, p)=\frac{1}{\pi \hbar} \int_{-\infty}^{\infty}\langle x+y|\rho| x+y\rangle e^{2 i p y / \hbar} d y .
$$

La transformación (o mapeo) de Wigner es la inversa de la transformación de Weyl (Zachos et al., 2005), que mapea el espacio de fases de funciones al espacio de Hilbert de operadores, en la cuantificación de Weyl.

La función de Wigner satisface las siguientes propiedades: 
1. $P(x, p)$ es real.

2. Las funciones de distribución de $x$ y $p$ están dadas por las marginales:

a) $\int_{-\infty}^{\infty} d p P(x, p)=\langle x|\rho| x\rangle$. (Para un estado puro se tiene $\int_{-\infty}^{\infty} d p P(x, p)=$ $\left.|\psi(x)|^{2}\right)$.

b) $\int_{-\infty}^{\infty} d x P(x, p)=\langle p|\rho| p\rangle$. (Para un estado puro se tiene $\int_{-\infty}^{\infty} d x P(x, p)=$ $\left.|\phi(p)|^{2}\right)$.

3. $\int_{-\infty}^{\infty} d x \int_{-\infty}^{\infty} d p P(x, p)=\operatorname{Tr}(\rho)=1$.

4. De las dos primeras propiedades se deduce que $P(x, p)$ puede ser negativa, a excepción del caso correspondiente a estados coherentes.

5. Posee las siguientes simetrías de reflexión:

a) Simetría temporal: $\psi(x) \rightarrow \psi(x)^{*} \Rightarrow P(x, p) \rightarrow P(x,-p)$.

b) Simetría espacial: $\psi(x) \rightarrow \psi(-x) \Rightarrow P(x, p) \rightarrow P(-x,-p)$.

6. Es invariante de Galileo $\psi(x) \rightarrow \psi(x+y)^{*} \Rightarrow P(x, p) \rightarrow P(x+y, p)$.

7. La superposición de dos funciones de onda se puede calcular como: $|\langle\psi \mid \theta\rangle|^{2}=\int_{-\infty}^{\infty} d x \int_{-\infty}^{\infty} d p P_{\psi}(x, p) P_{\theta}(x, p)$.

8. Los valores medios de los operadores se pueden calcular con sus respectivas transformaciones de Wigner:

a) $g(x, p) \equiv 2 \pi \hbar \int_{-\infty}^{\infty} d y\langle x-y / 2|G| x+y / 2\rangle e^{i p y / \hbar}$.

b) $\langle x|G| x\rangle=\int_{-\infty}^{\infty} \frac{d p}{h} e^{i p(x-y) / \hbar} g\left(\frac{x+y}{2}, p\right)$.

9. A partir de la desigualdad de Cauchy-Schwarz se puede ver que $-\frac{2}{h} \leq P(x, p) \leq$ $\frac{2}{h}$.

\subsubsection{Medición de la función de Wigner y estados del campo electro- magnético}

No es posible medir en forma directa la función de cuasi-distribución de Wigner. En su lugar se mide, experimentalmente para diferentes ángulos, la distribución 


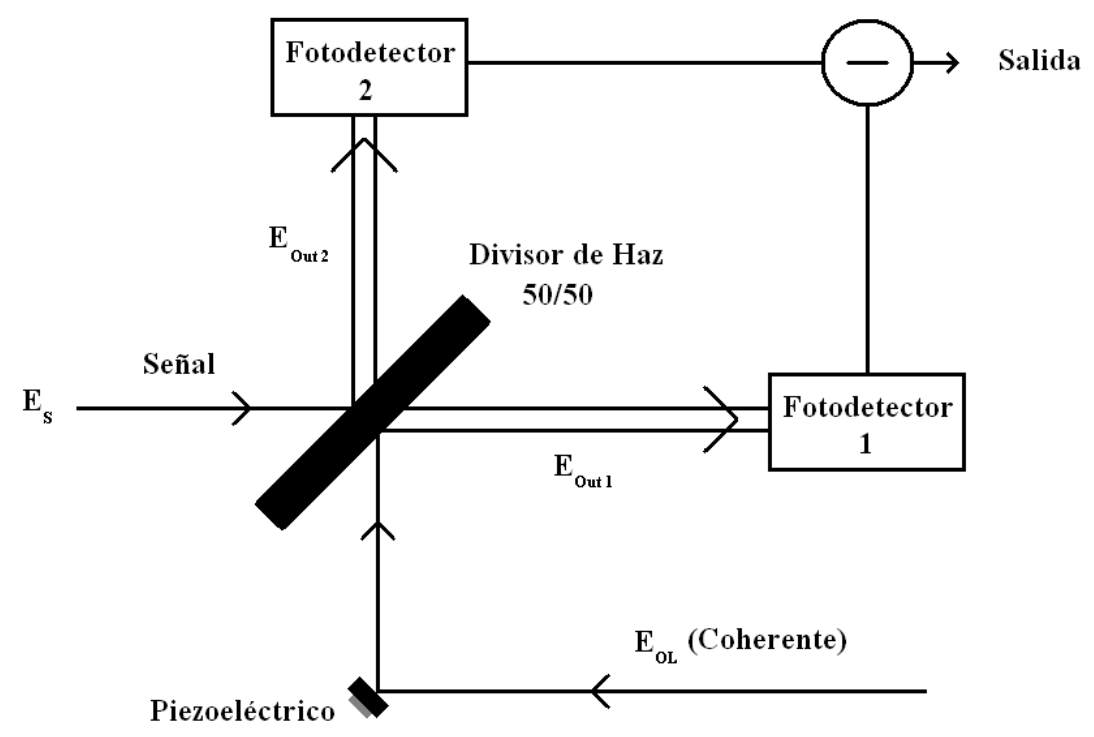

Figura 1.1: Esquema experimental para medir las cuadraturas del campo electromagnético.

marginal de una de las cuadraturas rotadas para luego reconstruir la función de Wigner del estado. Para esto, hay que tener en cuenta el siguiente resultado

$$
\tilde{p}(\xi, \theta)=\tilde{W}(\xi \cos \theta, \xi \sin \theta)
$$

El cual, nos dice que la función característica (transformada de Fourier) de una cuadratura rotada $\tilde{p}(\xi, \theta)$, coincide con la transformada de Fourier de la función de Wigner en coordenadas polares $\tilde{W}(\xi \cos \theta, \xi \sin \theta)$. Entonces, midiendo las distribuciones marginales (rotadas), se puede calcular su función característica y a partir de la misma la función de Wigner correspondiente.

En la Figura 1.1 se muestra un esquema experimental para medir las las cuadraturas del campo electromagnético. El funcionamiento básico es el siguiente: el haz de la señal $E_{s}$ (que se quiere medir) y un haz auxiliar $E_{O L}$ inciden en un divisor de haz y emergen de este dos haces $E_{O u t 1}$ y $E_{O u t 2}$. Podemos asociar a este esquema los operadores de destrucción del campo electromagnético $a, b, c$ y $d$, tales que:

$$
\begin{array}{rlrl}
E_{s} & \rightarrow a, & E_{\text {Out } 1} & \rightarrow c, \\
E_{O L} & \rightarrow b, & E_{\text {Out } 1} \rightarrow d .
\end{array}
$$


Los operadores de destrucción $c$ y d, para un divisor de haz 50/50, quedan relacionados con los incidentes de la siguiente forma

$$
c=\frac{1}{\sqrt{2}}(a-b), \quad d=\frac{1}{\sqrt{2}}(a+b) .
$$

Los fotodetectores 1 y 2 miden las intensidades, número medio de fotones, $\left\langle c^{\dagger} c\right\rangle$ y $\left\langle d^{\dagger} d\right\rangle$ de cada haz emergente. Un dispositivo electrónico realiza la diferencia de las intensidades en los detectores I. Analíticamente la diferencia de intensidades $I$ se escribe

$$
I=d^{\dagger} d-c^{\dagger} c=a^{\dagger} b+b^{\dagger} a
$$

Si el haz auxiliar es un estado coherente lo suficientemente intenso se pueden reemplazar los operadores $b$ y $b^{\dagger}$ por sus versiones clásicas $B e^{i(\omega t-\theta)}$ y $B e^{-i(\omega t-\theta)}$ y se obtiene

$$
I=B\left[a e^{i(\omega t-\theta)}+a^{\dagger} e^{-i(\omega t-\theta)}\right]
$$

Teniendo en cuenta la dependencia temporal de los operadores $a=a_{0} e^{-i \omega t}, a^{\dagger}=$ $a_{0}^{\dagger} e^{i \omega t}$ se obtiene

$$
I=B\left[a_{o} e^{-i \theta}+a_{o}^{\dagger} e^{i \theta}\right]
$$

que es proporcional a la cuadratura rotada $X_{\theta}=1 / \sqrt{2}\left(a_{o} e^{-i \theta}+a_{o}^{\dagger} e^{i \theta}\right)$. Para variar la fase relativa $\theta$, entre el haz de la señal y el haz auxiliar, se utiliza un espejo sujeto a un piezoeléctrico al que se lo somete a una corriente sinusoidal.

Los resultados de medir las cuadraturas del campo electromagnético, dado su carácter cuántico, son variables estadísticas. Esto es, variables que están distribuidas aleatoriamente de acuerdo con la probabilidad determinada por el estado del sistema. Un indicador de la precisión con la que se mide es su dispersión $\Delta X_{\theta}$. El valor mínimo del producto de las dispersiones que se obtienen al realizar dos medidas simultaneas está acotado inferiormente por al principio de incerteza. En la Figura 1.2(a) se muestran los resultados esquemáticos que se obtendrían al realizar medidas de las cuadraturas para un estado coherente del campo electromagnético. Estos estados presentan idéntica dispersión para sus cuadraturas y son la mínima 

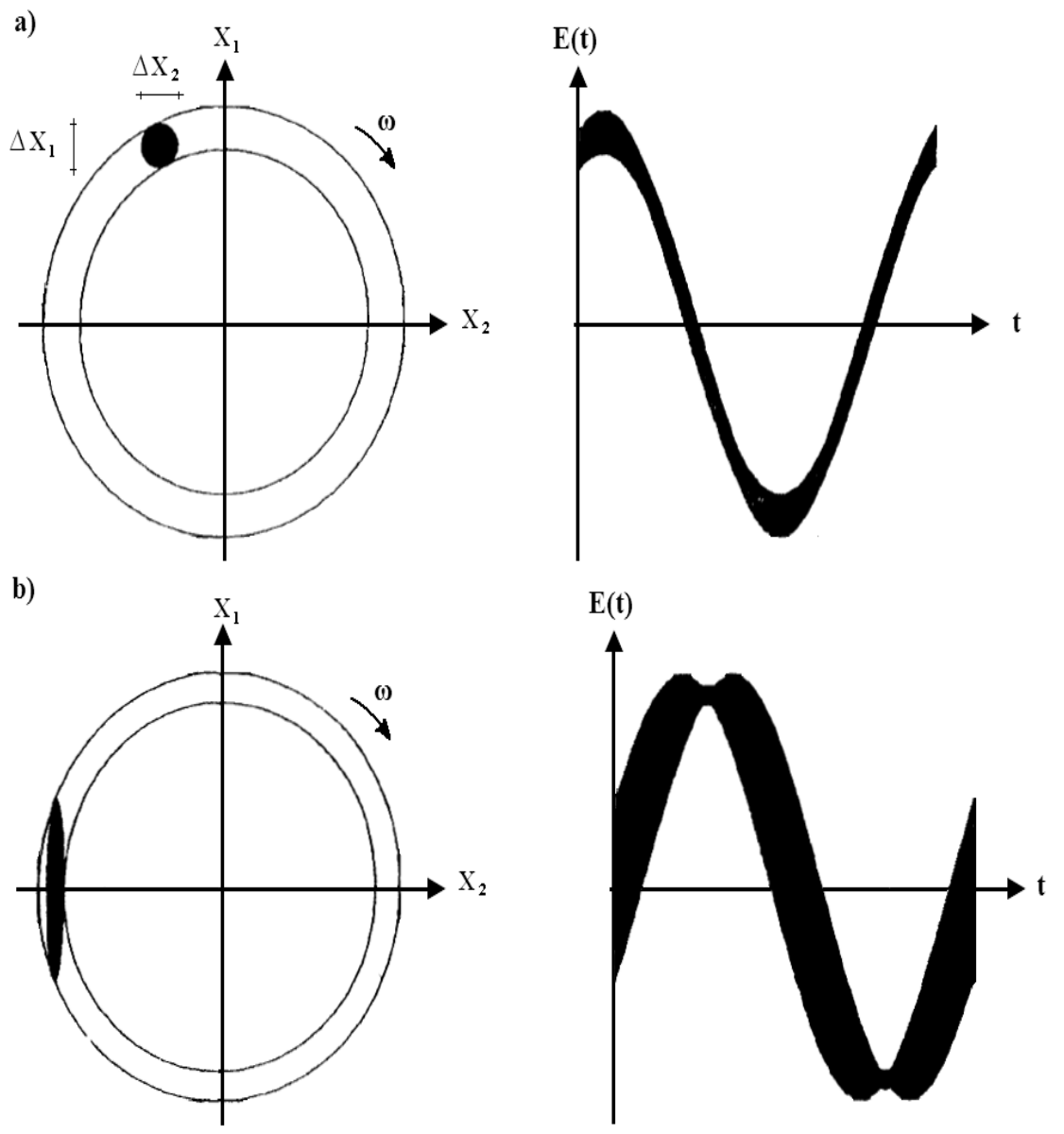

Figura 1.2: Cuadraturas y campo eléctrico en función del tiempo. a) Para un estado coherente. b) Para un estado que presenta compresión en incerteza.

posible, esto es $\Delta X_{1}=\Delta X_{2}=1$ (ver Apéndice B.2). Por otra parte, la Figura 1.2(b) muestra un estado que presenta reducción de incertezas en una de sus cuadraturas, a expensas del incremento en su conjugada (ver Apéndice B.3). Si la reducción de la incerteza en una de las variables es tal que toma un valor por debajo del límite cuántico estándar ${ }^{1}$ se dice que el estado se encuentra "comprimido" en incerteza. En la Figura 1.3 muestra un ejemplo de la reconstrucción de la función de Wigner a partir de las medidas del campo electromagnético.

\footnotetext{
${ }^{1}$ Ver Sección 1.3.
} 
a)

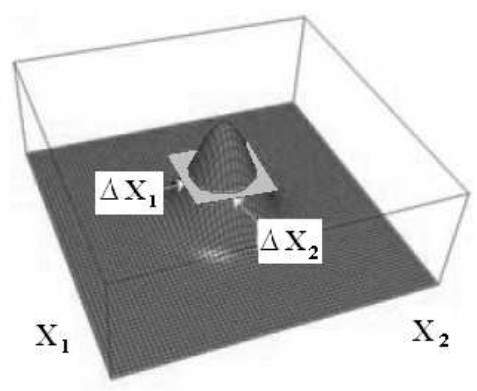

b)

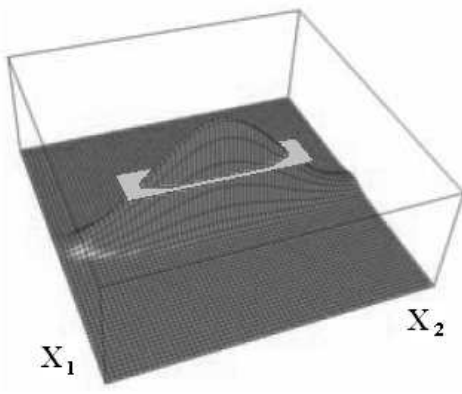

c)

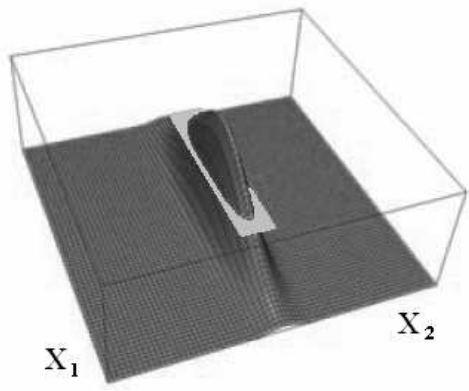

Figura 1.3: Función de Wigner para variables conjugadas $X_{1}$ y $X_{2}$. a)Función de Wigner para un estado coherente. b)Función de Wigner para estados comprimidos en incerteza en la variable $X_{1}$. c)Idem (b) en la variable $X_{2}$.

\subsection{Estados coherentes, representaciones y estados cohe- rentes de espín}

Los estados coherentes de un sistema cuántico han tenido una amplia utilización en distintos problemas físicos puesto que, bajo ciertas condiciones, pueden construirse con dispersión minima y en este sentido son los más "parecidos" a los estados clásicos. Una propiedad importante, que tienen estos estados, es que forman una base sobre completa y que no son ortogonales entre sí. Estas propiedades, más allá de la aparente dificultad para trabajar con ellos, brindan la posibilidad de describir sistemas cuánticos en forma realista. Por ejemplo se puede mencionar el caso del campo electromagnético (ver Sección B.2, del Apéndice B) en el cual un haz de láser, muy por encima del umbral, puede ser muy bien descripto por un estado coherente. En lo referido a esta tesis se utilizan estados coherentes del oscilador armónico, para representar estados de radiación electromagnética, y estados coherentes de espín.

A continuación se da la definición formal de los estados coherentes para un grupo en general, luego se muestran las representaciones de espín y se detalla la construcción de los estados coherentes de espín utilizada en esta tesis. La forma de construir 
los estados coherentes del oscilador armónico, y sus propiedades, se detallan en la Sección B.2 del Apéndice B.

\subsubsection{Estados coherentes}

Sea $T(g)$ una representación unitaria irreducible de un grupo de Lie $G$, que actúa sobre un espacio de Hilbert $H$ de un sistema cuántico:

$$
|\psi\rangle \in H, \quad T(g)|\psi\rangle=\left|\psi_{g}\right\rangle
$$

Se llama subgrupo estacionario del estado $\left|\psi_{0}\right\rangle$, al subgrupo $H_{0} \subset G$ si para todo $h \in H_{0}$ se tiene

$$
T(h)\left|\psi_{0}\right\rangle=e^{i f(h)}\left|\psi_{0}\right\rangle
$$

Si $H_{0}$ es el subgrupo estacionario del estado $\left|\psi_{0}\right\rangle$ es obvio que es autoestado para todos los generadores del subgrupo $H_{0}$. El álgebra de Lie correspondiente al subgrupo $H_{0}$ se la llama subalgebra estacionaria del estado $\left|\psi_{0}\right\rangle$.

Debido a que los estados cuánticos están definidos a menos de una fase, los elementos $\left|\psi_{g}\right\rangle=T(g)|\psi\rangle$ pueden ponerse en correspondencia con los elementos del espacio cociente $G / H_{0}$.

Si se tienen todos estos elementos en cuenta se puede enunciar:

Definición: Se llama sistema de estados coherentes del grupo $G$ respecto del vector fundamental $\left|\psi_{0}\right\rangle$ al conjunto de vectores $\left\{\left|\xi_{g}\right\rangle\right.$ tal que $\left|\xi_{g}\right\rangle=T(\tilde{g})\left|\psi_{0}\right\rangle$ con $\tilde{g} \in$ $\left.G / H_{0}\right\}$

En principio los estados coherentes se pueden generar a partir de cualquier estado $\left|\psi_{0}\right\rangle \in H$. Sin embargo, se puede mostrar que los estados $\left|\psi_{0}\right\rangle$ que tienen el subgrupo estacionario más grande, generan estados coherentes que están mas cerca de los estados clásicos en el sentido que ellos minimizan las relaciones de incerteza y, para el caso de álgebras compactas, también minimizan las fluctuaciones del Casimir cuadrático (Zhelobenko, 1973). 


\subsubsection{Representaciones de momento angular}

El momento angular $\mathbf{J}$ es un operador vectorial, y como tal tiene tres componentes $J_{x}, J_{y}, J_{z}$. Las relaciones de conmutación cíclicas entre las componentes que definen un momento angular como tal son:

$$
\left[J_{i}, J_{j}\right]=i \epsilon_{i j k} J_{k}
$$

donde los sub-índices $i, j, k$ denotan cualquiera de las tres componentes en direcciones ortogonales y $\epsilon_{i j k}$ es el símbolo de Levi-Civita. El operador

$$
\mathbf{J}^{2}=\mathbf{J} \cdot \mathbf{J}=J_{x}^{2}+J_{y}^{2}+J_{z}^{2}
$$

representa el cuadrado del operador momento angular $\mathbf{J}$ y conmuta con las tres componentes del momento angular $J_{x}, J_{y}, J_{z}$. Debido a esto, la base de autoestados de un sistema que involucre como variable un operador de momento angular, se puede expresar en términos de los autovalores de $\mathbf{J}^{2}$ y $J_{z}$, como $|J, M\rangle$. Estos estados satisfacen

$$
\mathbf{J}^{2}|J, M\rangle=J(J+1)|J, M\rangle, \quad J_{z}|J, M\rangle=M|J, M\rangle,
$$

donde los valores de $M$, que son $(2 J+1)$, van desde $-J$ a $J$, con $J$ entero o fraccionario.

Por medio de combinaciones lineales de los operadores de espín se pueden definir los operadores no-Hermíticos $J_{ \pm}$como

$$
J_{ \pm}=J_{x} \pm i J_{y}
$$

los cuales se llaman operadores escalera, debido a que al aplicarlos sobre $|J, M\rangle$ dan como resultado

$$
J_{ \pm}|J, M\rangle=\sqrt{(J \mp M)-(J \pm M+1)}|J, M \pm 1\rangle .
$$

Estos son análogos a los operados de creación y destrucción del oscilador armónico y satisfacen las siguientes relaciones de conmutación

$$
\left[J_{+}, J_{-}\right]=2 J_{z} \quad\left[J_{z}, J_{ \pm}\right]= \pm J_{ \pm} .
$$


A partir de estas, es simple probar las siguientes relaciones

$$
\mathbf{J}^{2}=J_{z}^{2}+\left(J_{+} J_{-}+J_{-} J_{+}\right), \quad J_{ \pm} J_{\mp}=\mathbf{J}^{2}-J_{z}\left(J_{z} \pm 1\right) .
$$

Utilizando los operadores escalera $J_{ \pm}$podemos derivar todos los estados para un dado $J$ aplicándolos en forma sucesiva a los estados de mayor (o menor) proyección de momento angular $|J, J\rangle(|J,-J\rangle)$. Esto es

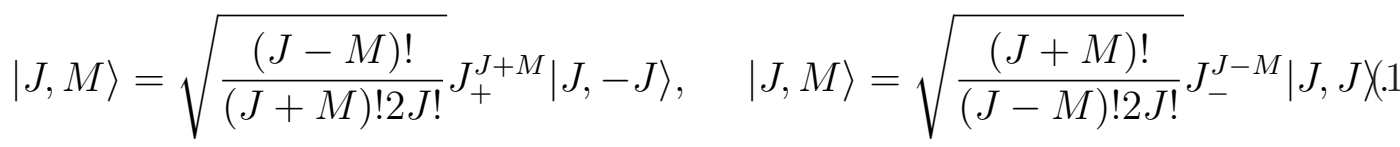

Las relaciones de incerteza asociadas a las relaciones de conmutación (1.13) son:

$$
\left\langle\Delta J_{i}^{2}\right\rangle\left\langle\Delta J_{j}^{2}\right\rangle \geq \frac{1}{4}\left|\left\langle J_{k}\right\rangle\right|^{2}
$$

\subsubsection{Esfera de Bloch}

Para un sistema de dos niveles una superposición arbitraria de estados puede escribirse como

$$
|\psi\rangle=c_{0}|0\rangle+c_{1}|1\rangle
$$

donde $c_{0}$ y $c_{1}$ son constantes complejas. La condición de normalización requiere

$$
\left|c_{0}\right|^{2}+\left|c_{1}\right|^{2}=1
$$

lo que indica que es posible representar al estado por un vector de longitud unidad. A esta representación, se la conoce como representación de Bloch y sirve para dar una interpretación geométrica de una superposición coherente de estados. Al vector que define el estado y a la esfera que este define, se los llaman vector y esfera de Bloch respectivamente. La representación de Bloch fue originalmente desarrollada por Felix Bloch en 1946 para describir el fenómeno de resonancia magnética nuclear, y posteriormente fue adaptado por Feynman et al. (1957) para sistemas de dos niveles. La dirección del vector de Bloch puede especificar en coordenadas Cartesianas $(x, y, z)$ o coordenadas esféricas $(r, \theta, \phi)$. Dado que el vector debe tener norma uno $r=x^{2}+y^{2}+z^{2}=1$ solo se necesitan dos variables independientes para definir un estado sobre la esfera de Bloch. Una parametrización posible es utilizando los 


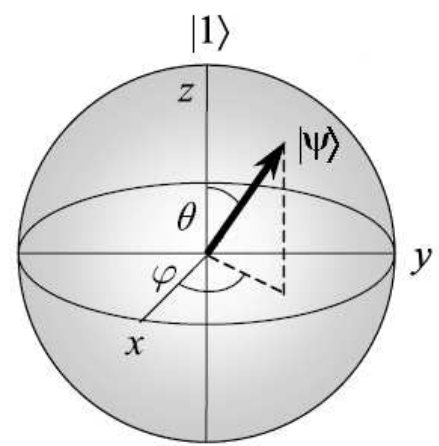

$|0\rangle$

Figura 1.4: Representación de la esfera de Bloch para un sistema de dos niveles $|0\rangle,|1\rangle$ (espín 1/2).

ángulos $(\theta, \phi)$. Esto permite realizar un mapeo entre las amplitudes $\left(c_{0}, c_{1}\right)$ y las direcciones del vector de Bloch.

La conexión entre el vector de Bloch y la función de onda puede ser hecha si se definen las direcciones del polo sur y el polo norte, como los estados $|0\rangle$ y $|1\rangle$ respectivamente, como se muestra en la Figura 1.4. Así el estado fundamental $|\psi\rangle=|0\rangle$ corresponde al punto $(0,0,-1)$ en cordenadas Cartesianas, o $\theta=\pi$ en coordenadas polares. Analogamente $|\psi\rangle=|1\rangle$ corresponde al punto $(0,0,1)$ o $\theta=0$. Un estado arbitrario en coordenadas Cartesianas esta dado por

$$
\begin{gathered}
x=2 \operatorname{Re}\left\langle c_{0} c_{1}\right\rangle, \\
y=2 \operatorname{Im}\left\langle c_{0} c_{1}\right\rangle, \\
z=\left|c_{1}\right|^{2}-\left|c_{0}\right|^{2},
\end{gathered}
$$

y en coordenadas polares se obtiene

$$
\begin{gathered}
c_{0}=\sin (\theta / 2), \\
c_{1}=e^{i \phi} \cos (\theta / 2) .
\end{gathered}
$$

A continuación se presentarán los estados coherentes de espín. Como se mostrará estos estados pueden ser caracterizados por un vector inicial y dos parámetros, por lo cual puede hacerse una correspondencia entre estos estados y la esfera de Bloch. 


\subsubsection{Estados coherentes de espín}

Los estados coherentes de espín, o estados coherentes de $S U(2)$, fueron introducidos a principio de los años setenta por Radcliffe(1971), Gilmore(1972a)(1972b), y Perelomov(1972). Como se comento en la Sección 1.2.1 para obtener una familia de estados coherentes $\left\{G,\left|\psi_{0}\right\rangle\right\}$ hay que aplicar los operadores de la representación $T^{J}(\tilde{g})^{2}$, con $\tilde{g} \in G / H_{0}$, a un vector fijo $\left|\psi_{0}\right\rangle$. Para el grupo $\mathrm{SU}(2)$ los elementos $T^{J}(g)$ puede ser escrito como $^{3}$

$$
T^{J}(g)=T^{J}\left(g_{n}\right) T^{J}(h), \quad h \epsilon H_{0}=U(1) .
$$

Así, si se toma un vector $|J, M\rangle$ como el vector fundamental $\left|\psi_{0}\right\rangle$, el estado coherente está determinado por el vector unitario $\mathbf{n}(\theta, \phi)=(\sin \theta \cos \phi, \sin \theta \sin \phi, \cos \theta)$ en la esfera bi-dimensional $S^{2}=S U(2) / U(1)$

$$
|n\rangle=e^{i \alpha(n)} T\left(g_{n}\right)\left|\psi_{0}\right\rangle
$$

El factor de fase $e^{i \alpha(n)}$ puede ser elegido igual a la unidad, así el sistema de estados coherente de $\mathrm{SU}(2)$ tiene la forma

$$
|n\rangle=D(n)\left|\psi_{0}\right\rangle
$$

El operador $D(n)$ puede ser escrito como

$$
D(n)=T^{J}\left(g_{n}\right)=e^{i \theta(\mathbf{m J})} \text {, con } 0 \leq \theta<\pi
$$

y $\mathbf{m}$ es el vector unidad, ortogonal a $\mathbf{n}$ y a $\mathbf{n}_{\mathbf{0}}=(\mathbf{0}, \mathbf{0}, \mathbf{1})$, luego $\mathbf{m}=(\sin \phi,-\cos \phi, \mathbf{0})$. Esta definición de $\mathbf{m}$ es válida para todo $\mathbf{n}$, excluyendo el polo sur $\mathbf{n}=(\mathbf{0}, \mathbf{0}, \mathbf{- 1})$. Otra forma de escribir el operador $D(n)$, que es análoga a los operadores de desplazamiento del oscilador armónico (Ecuación (B.8)), es

$$
D(n)=D(\xi)=e^{\left(\xi J_{+}-\bar{\xi} J^{-}\right)}, \quad \text { con } \xi=i \frac{\theta}{2}\left(m_{1}-i m_{2}\right)=-|\xi| e^{-i \phi}
$$

\footnotetext{
${ }^{2}$ Para el caso de espín el supra índice $J$ fija la representación irreducible de espín.

${ }^{3}$ Cada elemento del grupo SU(2) puede ser representado de la forma $g=\tilde{g} h$ donde $h \in H=U(1)$ es el subgrupo estacionario de todos los estados de una representación no trivial de $\mathrm{SU}(2)$ formado por las matrices diagonales $\operatorname{del}$ tipo $\left(\begin{array}{cc}e^{-i \psi / 2} & 0 \\ 0 & e^{i \psi / 2}\end{array}\right)$ y $\tilde{g}$ es un elemento $\operatorname{del} \operatorname{coset} S U(2) / U(1)$ que tiene la forma $\left[\begin{array}{c}a \\ b^{*}-b \\ a\end{array}\right]$, con $a^{2}+|b|^{2}=1$ y $a>0$ donde $a=\cos (\theta / 2)$ y $b=e^{\phi / 2} \sin \theta / 2$ Esto muestra que el espacio cociente $S U(2) / U(1)$ es isomorfo a la esfera de $S^{2}$
} 
Este operador puede ser escrito en la forma "normal" como

$$
D(\xi)=e^{\zeta J_{+}} e^{\zeta J_{0}} e^{\bar{\zeta} J_{-}}
$$

con

$$
\zeta=-\tan \frac{\theta}{2} e^{-i \phi}, \quad \eta=-2 \ln (\cos |\xi|)+\ln \left(1+|\zeta|^{2}\right) .
$$

Cualquier vector de la representación $|J, M\rangle$ puede ser utilizado como vector fundamental $\left|\psi_{0}\right\rangle$. A primera vista, todos los sistemas de estados coherentes son equivalentes, sin embargo, dado que, para los vectores $|J, \pm J\rangle$ la dispersión cuadrática del operador $\mathbf{J}^{\mathbf{2}}=\mathbf{J}_{\mathbf{1}}^{\mathbf{2}}+\mathbf{J}_{\mathbf{2}}^{\mathbf{2}}+\mathbf{J}_{\mathbf{3}}^{\mathbf{2}}$ (Casimir cuadrático) es mínima

$$
\Delta \mathbf{J}^{2}=\left(\Delta \mathbf{J}^{2}\right)_{\min }=J,
$$

los sistemas de estados coherentes que se generan a partir de estos, que son idénticos entre sí, estarán mas cerca de los estados clásicos (Zhelobenko, 1973), dado que tienen dispersión mínima.

$$
\begin{aligned}
|\theta, \phi\rangle \equiv|\zeta\rangle & =\frac{1}{\left(1+|\zeta|^{2}\right)^{J}} e^{\left(\zeta J_{+}\right)}|J,-J\rangle \\
& =\frac{1}{\left(1+|\zeta|^{2}\right)^{J}} \sum_{k=0}\left(\frac{\zeta^{k}}{k !} J_{+}^{k}\right)|J,-J\rangle \\
& =\sum_{M=-J}^{J} \sqrt{\frac{(2 J) !}{(J+M) !(J-M) !}} \frac{\zeta^{J+M}}{\left(1+|\zeta|^{2}\right)^{J}}|J, M\rangle
\end{aligned}
$$

Donde para etiquetar los estados coherentes en términos de las variables angulares, se utilizo la transformación $\zeta=\tan \frac{\theta}{2} e^{\phi}$ que lleva los elementos de la esfera de Riemman sobre los complejos de norma uno $(\theta / 2, \phi)$ respecto del polo sur

$$
S^{2} \text { э } r(\theta, \phi)=(x, y, z) \rightarrow \zeta=\frac{x+i y}{1+z} \in \mathbb{C} \Leftrightarrow\left\{\begin{array}{l}
x=\frac{2 \operatorname{Re}(\zeta)}{1+|\zeta|^{2}} \\
y=\frac{2 \operatorname{Im}(\zeta)}{1+|\zeta|^{2}} \\
z=\frac{1-|\zeta|^{2}}{1+|\zeta|^{2}}
\end{array}\right.
$$

En términos de la base $|J, M\rangle$ un estado coherente de espín $|\theta, \phi\rangle$ puede ser escrito en variables angulares como (Perelomov, 1972; Radcliffe, 1971)

$$
|\theta, \phi\rangle=\sum_{M=-J}^{J} \sqrt{\frac{(2 J) !}{(J+M) !(J-M) !}}\left(\cos \frac{\theta}{2}\right)^{J+M}\left(\sin \frac{\theta}{2}\right)^{J-M} e^{i(J-M) \theta}|J, M\rangle .
$$


Algunas propiedades importantes que tienen los estados coherentes de espín son:

1. El sistema de estados coherentes de espín es completo.

2. Los estados coherentes no son ortogonales entre sí

$$
\begin{gathered}
\langle\xi \mid \eta\rangle=\left[\left(1+|\xi|^{2}\right)\left(1+|\eta|^{2}\right)\right]^{-j}(1+\bar{\xi} \eta)^{2 j} \\
|\langle\xi \mid \eta\rangle|^{2}=\left(\frac{(1+\bar{\xi} \eta)(1+\xi \bar{\eta})}{\left.\left(1+|\xi|^{2}\right)\left(1+|\eta|^{2}\right)\right]}\right)^{2 j} .
\end{gathered}
$$

3. En la base de estados coherentes la identidad se puede escribir como

$$
\frac{2 j+1}{4 \pi} \int d \mathbf{n}|n\rangle\langle n| \text {. }
$$

4. Los estados coherentes son autoestados del operador $\mathbf{J}$ en la dirección del vector unitario $\mathbf{n}$, esto es

$$
(\mathbf{J} . \mathbf{n})|\theta, \phi\rangle=-J|\theta, \phi\rangle .
$$

Esto es así ya que se utilizó como vector fundamental $\left|\psi_{0}\right\rangle=|J, \pm J\rangle$, por lo que $|\theta, \phi\rangle=D(n)\left|\psi_{0}\right\rangle$ y además $D(n) J_{x} D(n)^{-1}=(\mathbf{J} . \mathbf{n})$.

5. Los estados coherentes minimizan la relación de incertidumbre de Heisemberg ya que

$$
\left\langle J_{1}^{2}\right\rangle\left\langle J_{2}^{2}\right\rangle \geq \frac{1}{4}\left\langle J_{3}\right\rangle^{2}
$$

se satura con la elección del vector $\left|\psi_{0}\right\rangle=|J, \pm J\rangle$. Además para los operadores de momento angular rotados $J_{k}^{\prime}=D(n) J_{k} D^{-1}(n)$ se tiene que

$$
\left\langle J_{1}^{\prime 2}\right\rangle\left\langle J_{2}^{\prime 2}\right\rangle \geq \frac{1}{4}\left\langle J_{3}^{\prime}\right\rangle^{2}
$$

se minimiza para los estados coherentes de espín.

6. Los valores medios de los operadores de espín para un estado coherente vale

$$
\left\langle\zeta\left|J_{0}\right| \zeta\right\rangle=-j \frac{1-|\zeta|^{2}}{1-|\zeta|^{2}}, \quad\left\langle\zeta\left|J_{+}\right| \zeta\right\rangle=2 j \frac{\bar{\zeta}}{1-|\zeta|^{2}} .
$$

En términos de las variables angulares el valor medio de las componentes de $\mathbf{J}$ se pueden escribir como

$$
\langle\theta, \phi|\mathbf{J}| \theta, \phi\rangle=(-J \sin \theta \cos \phi,-J \sin \theta \sin \phi,-J \cos \theta)=-J \mathbf{n} .
$$


La Ecuación (1.46) define al vector de Bloch colectivo, que está formado por los valores medios de los operadores $J_{i}$ en la dirección del momento angular clásico. Los valores esperados de los operadores $J_{i}$ son idénticos a los que se obtienen para el caso de $J=1 / 2$ con la única diferencias que hay que cambiar $1 / 2 \rightarrow J$. Esto sugiere que el estado coherente $|\theta, \phi\rangle$ puede ser interpretado como una generalización de un estado de dos niveles. Las fluctuaciones cuadráticas de cada operador de espín en un estado coherente $|\theta, \phi\rangle$ se escriben

$$
\begin{aligned}
\left(\Delta J_{x}\right)^{2} & =\frac{J}{2}\left(1-\sin ^{2} \theta \cos ^{2} \phi\right) \\
\left(\Delta J_{y}\right)^{2} & =\frac{J}{2}\left(1-\sin ^{2} \theta \sin ^{2} \phi\right) \\
\left(\Delta J_{z}\right)^{2} & =\frac{J}{2}\left(1-\cos ^{2} \theta\right)
\end{aligned}
$$

Además se tiene

$$
\left(\Delta J_{x}\right)^{2}+\left(\Delta J_{y}\right)^{2}+\left(\Delta J_{z}\right)^{2}=\left\langle\mathbf{J}^{2}\right\rangle-\langle\mathbf{J}\rangle^{2}=J
$$

También es posible calcular las fluctuaciones del operador $\mathbf{J}$ en una dirección perpendicular al del espín medio n. Para esto, se puede escribir

$$
\mathbf{J}=\mathbf{n}(\mathbf{J} \cdot \mathbf{n})+\mathbf{J}_{\perp}
$$

donde $\mathbf{J}_{\perp}$ se puede escribir como la suma de dos vectores perpendiculares entre sí que están en el plano ortogonal a $\mathbf{n}$

$$
\mathbf{J}_{\perp}=\mathbf{J}_{\perp}^{1}+\mathbf{J}_{\perp}^{2}, \quad \mathbf{J}_{\perp}^{1} \perp \mathbf{J}_{\perp}^{2}
$$

Teniendo en cuenta la Ecuación (1.46), es simple ver que

$$
\left\langle\theta, \phi\left|\mathbf{J}_{\perp}\right| \theta, \phi\right\rangle=0
$$

y si despejamos $\mathbf{J}_{\perp}$ de la Ecuación (1.51) se puede calcular

$$
\left\langle\left(\Delta \mathbf{J}_{\perp}\right)^{2}\right\rangle=\left\langle\mathbf{J}^{2}\right\rangle-\langle\mathbf{J} \cdot \mathbf{n}\rangle^{2}=J(J+1)-J^{2}=J
$$

De la Ecuación (1.34) se puede concluir que, para los estados coherentes, las fluctuaciones de las componentes de $\mathbf{J}_{\perp}$ son iguales. Esta afirmación es simple de ver para 
el caso de que se tome un estado coherente $\operatorname{con} \theta=0$. Dado que las propiedades físicas no dependen de las rotaciones, estas propiedades no pueden cambiar por la aplicación de $D(\theta, \phi)$. Así se obtiene que

$$
\left\langle\theta, \phi\left|\left(\Delta \mathbf{J}_{\perp}^{\mathbf{1}}\right)^{2}\right| \theta, \phi\right\rangle=\left\langle\theta, \phi\left|\left(\Delta \mathbf{J}_{\perp}^{\mathbf{2}}\right)^{2}\right| \theta, \phi\right\rangle=\frac{J}{2}
$$

Teniendo en cuenta esto junto con la Ecuación (1.46) podemos visualizar un estado coherente de espín como un segmento simétrico de radio $\sqrt{J}$ sobre la esfera de Bloch de radio $J$, y centrado por el punto determinado por el vector $\mathbf{n}$. En el límite clásico, cuando $J \rightarrow \infty$ el cociente entre este segmento y el radio de la esfera de Bloch tiende $1 / \sqrt{J} \rightarrow 0$. Lo que significa que las fluctuaciones perpendiculares al vector de Bloch desaparecen.

\subsection{Relaciones de Incerteza y Squeezing}

En general, se denomina squeezing a la compresión de la incerteza de una variable cuántica, respecto a otra, por debajo de un "cierto límite". Para especificar este límite, sean dos operadores $A, B$, de un espacio de Hilbert. Para cualquier estado del sistema se satisface el principio de incertidumbre de Heisenberg

$$
(\Delta A)^{2}(\Delta B)^{2} \geq \frac{1}{4}|\langle[A, B]\rangle|^{2} .
$$

Donde $\Delta O$, es la varianza $\left((\Delta O)^{2}=\left\langle O^{2}\right\rangle-\langle O\rangle^{2}\right)$ del operador $O$ en un dado estado, y representa la incerteza cuántica mínima que tendrá un operador al ser medida. Como es evidente, esta desigualdad pone una cota inferior al producto de incertezas de realizar la medida de dos operadores en forma simultánea. La cota del producto de incertezas de dos operadores está dada por el valor medio del conmutador de los mismos. A partir de esta desigualdad, podemos clasificar la incerteza de un estado para un dado operador $A$ respecto a la incerteza que presenta otro operador $B$. Un estado que satura la desigualdad, esto es,

$$
(\Delta A)^{2}(\Delta B)^{2}=\frac{1}{4}|\langle[A, B]\rangle|^{2}
$$

se dirá de mínima incerteza. Se llama "límite cuántico estándar" al valor de incerteza que dos operadores pueden tomar en simultaneo, para un estado de minima 
incerteza, esto es

$$
(\Delta A)^{2}=(\Delta B)^{2}=\frac{1}{2}|\langle[A, B]\rangle|
$$

Un estado se dirá que esta "comprimido" en incerteza en el operador $A$, respecto a $B$ si el valor de incerteza de dicho operador es menor que el límite cuántico estándar

$$
(\Delta A)^{2}<\frac{1}{2}|\langle[A, B]\rangle|
$$

Es claro que la compresión de la incerteza en el operador $A$, en un cierto estado, se produce a expensas de que se incremente la incerteza en el operador $B$, para no violar el principio de incertidumbre de Heisenberg.

Un parámetro que da cuenta de esta compresión, en incerteza de un operador respecto a otro, es el llamado parámetro de squeezing que se define a partir del límite cuántico estándar

$$
\xi^{2}(A, B)=\frac{2(\Delta A)^{2}}{|\langle[A, B]\rangle|} .
$$

Se dirá que un cierto estado esta comprimido en incerteza en el operador $A$, respecto al operador $B$, si $\xi \leq 1$. Esta es una definición general que depende de las relaciones de conmutación de los operadores. Es obvio que, para un dado sistema, existen muchas posibilidades de definir un parámetro de squeezing dadas las posibles relaciones de conmutación entre operadores del sistema.

Si tomamos como ejemplo los estados del campo electromagnético (ver Apéndice B.3), dada la simplicidad de las relaciones de conmutación de los operadores involucrados (Grupo $U(1)$ ), es simple clasificar los estados según la incerteza de cada uno. Si se utilizan como operadores relevantes del sistema las cuadraturas del campo electromagnético (Ecuación (B.23)), la relación de conmutación y el principio de incerteza asociado a esta se escribe

$$
\left[X_{1}, X_{2}\right]=2 i, \quad \Delta X_{1} \Delta X_{2} \geq \frac{1}{2}\left|\left\langle\left[X_{1}, X_{2}\right]\right\rangle\right|=1 .
$$

Por lo cual, el límite cuántico estándar (Ecuación (1.56)) para este es una constante y el factor de squeezing se puede definir como (Ecuación (1.57))

$$
\xi^{2}\left(X_{1,2}, X_{2,1}\right)=\left(\Delta X_{1,2}\right)^{2}
$$




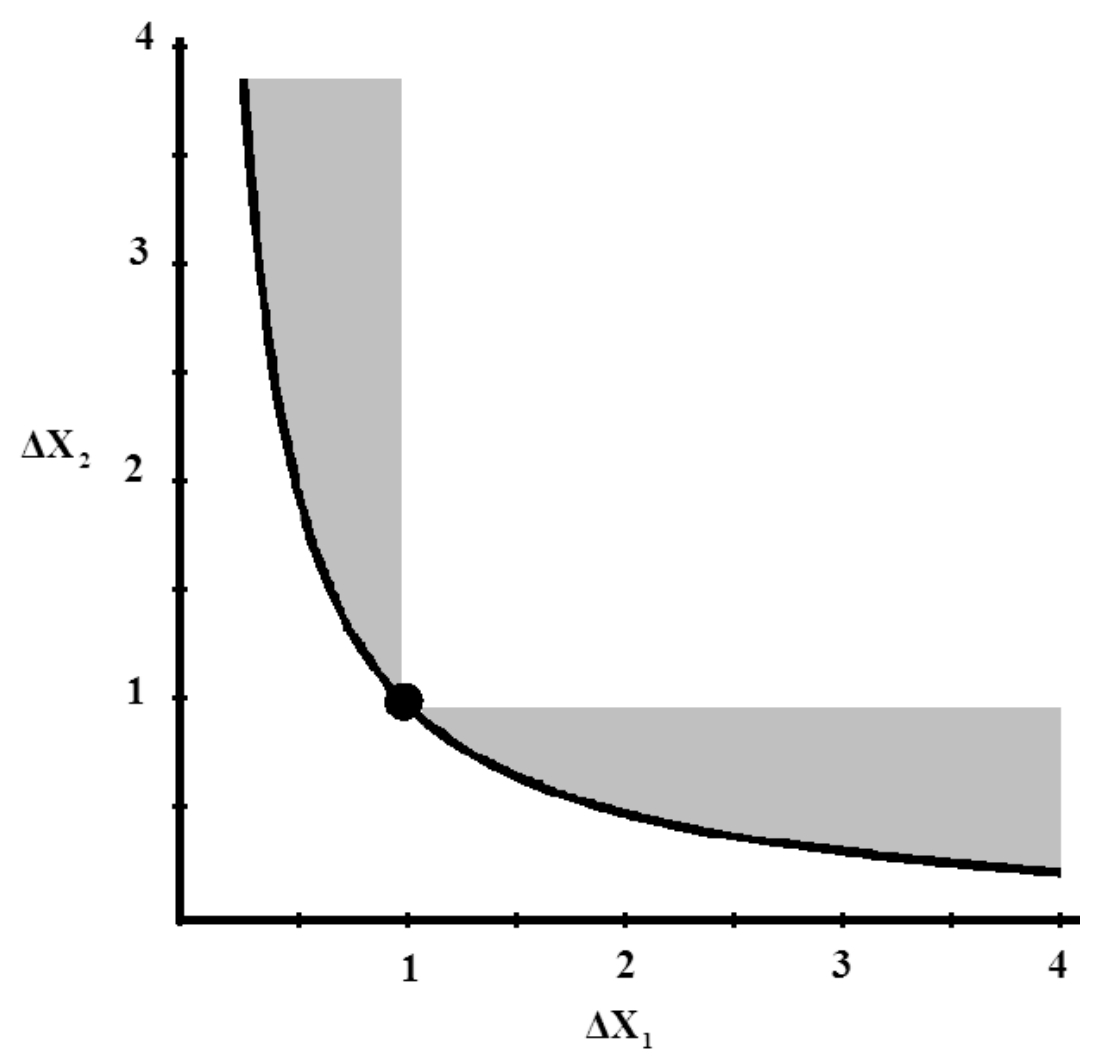

Figura 1.5: Gráfico de las incertezas de las cuadraturas del campo electromagnético $\Delta X_{1}$ versus $\Delta X_{2}$. Los puntos a la izquierda de la curva $\Delta X_{1} \Delta X_{2}=1$ representan estados no-físicos puesto que no satisfacen el principio de incertidumbre de Heisenberg. El punto $(1,1)$ representa a los estados coherentes $|\alpha\rangle$ (Ecuación (B.14)), mientras la región sombreada corresponde a estados comprimidos $|\alpha, \varepsilon\rangle$ (Ecuación (B.32)).

A partir de esta definición los estados del campo electromagnético pueden clasificarse según su incerteza (Figura 1.5). El punto $(1,1)$ se corresponde con los estados que presentan igual valor de la varianza de los operadores $X_{1}$ y $X_{2}$, esto es los estados coherentes del campo electromagnético (Ecuación (B.14)). Las zonas sombreadas representan estados comprimidos en una de las cuadraturas por debajo del límite cuántico estándar. En el caso del espín, dadas las relaciones de conmutación de los operadores intervinientes $(S U(2))$, existen varias posibilidades de definir squeezing y cada una de ellas será relevante (o no) dependiendo del contexto que se analice. A continuación se discutirá algunas de las definiciones frecuentes en la literatura y 
se prestará especial atención a la introducida por Kitaguawa y Ueda (1993).

\subsubsection{Squeezing en sistemas atómicos}

Si bien es posible definir la compresión en incerteza de una de las componentes de espín, en función de las otras, a partir de la las relaciones de conmutación de los operadores de espín

$$
\Delta J_{i} \Delta J_{j} \geq \frac{1}{2}\left|\left\langle J_{k}\right\rangle\right|
$$

de la misma forma que se menciono en la Sección 1.3, diciendo que el sistema de espines esta comprimido en incerteza en el operador $J_{i}$ respecto de la componente $J_{j}$ si

$$
\left(\Delta J_{i}\right)^{2}<\frac{1}{2}\left|\left\langle J_{k}\right\rangle\right|
$$

se tiene para esta definición varios puntos cuestionables. Dado que la relación de conmutación de los operadores de espín no es una constante, como en el caso del campo electromagnético, es necesario una hipótesis adicional para fijar el límite cuántico estándar. Ya que si no se lo hace, un estado coherente de espín rotado de forma conveniente estaría comprimido en incerteza respecto a la definición de la Ecuación (1.62), lo cual no es correcto, en el sentido que se explica a continuación. Como se vio en la Sección 1.2.4, si un sistema de espín está preparado en un estado coherente la fluctuación cuadrática de la componente de $\mathbf{J}$ en cualquier dirección perpendicular al vector $\langle\mathbf{J}\rangle$ es igual a $J / 2$. Físicamente se puede pensar a un estado coherente como la superposición de espines que se encuentran en el mismo estado (Figura 1.6(a)), así las fluctuaciones cuadráticas serán simplemente la suma de las fluctuaciones de cada espín. Uno puede decir que los espines no están correlacionados en el sentido de que el valor medio de cualquier observable se puede calcular como el promedio sobre los estados individuales de cada átomo. Dado que rotar un estado coherente nos devuelve otro estado coherente, volveremos a la misma situación en la que la incerteza se encontrará en el plano perpendicular al vector medio del espín y será isotrópica en dicho plano. En ese sentido es que la definición de la Ecuación (1.62) es cuestionable ya que para la misma situación de incerteza indica que el 

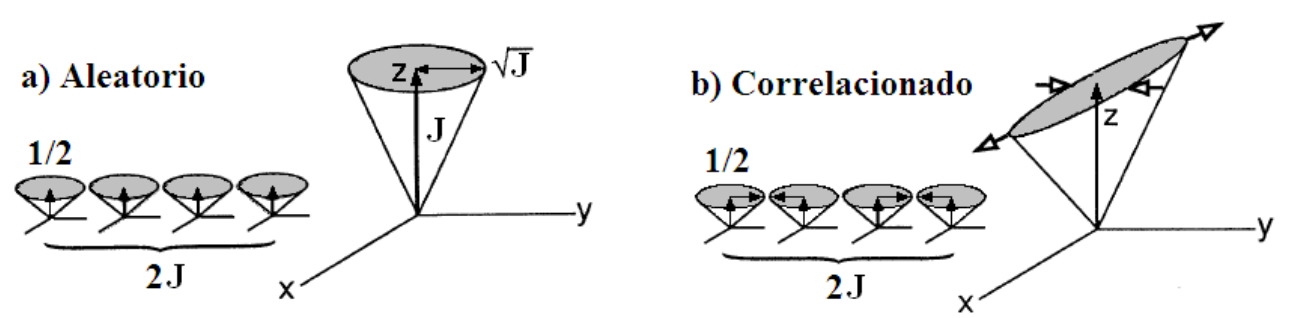

Figura 1.6: Representación esquemática de un espín $J$ en término de $2 J$ espines individuales 1/2.

a) Estado coherente de espín construido a partir de $2 J$ espines $1 / 2$ no-correlacionados. b) Estado comprimido de espín construido a partir de $2 J$ espines $1 / 2$ correlacionados.

estado está comprimido, y además no sirve como parámetro que de cuenta de las correlaciones entre los componentes del sistema.

\subsubsection{Factor de squeezing}

La idea básica de un sistema de espines comprimido en incerteza, es un conjunto correlacionados de espines de forma que las fluctuaciones no estén uniformemente distribuidas sobre el plano perpendicular al vector espín medio y en algunas direcciones sea menor a $J / 2$, a expensas de que aumenten en otra dirección (Figura $1.6(b))$.

Para correlacionar un sistema de espines entre ellos se necesita una transformación generada por una combinación no lineal de los generadores de $\mathrm{SU}(2)$, ya que una combinación lineal solo rotará los espines y no producirá correlaciones. Una forma de introducir correlaciones en un sistema colectivo de espines consiste en introducir fases no triviales en la Ecuación (1.38) que dependan de un conjunto de parámetros $\mu$, esto es

$|\theta, \phi, \mu\rangle=\sum_{M=-J}^{J} \sqrt{\frac{(2 J) !}{(J+M) !(J-M) !}}\left(\cos \frac{\theta}{2}\right)^{J+M}\left(\sin \frac{\theta}{2}\right)^{J-M} e^{i(J-M) \theta} e^{-i f(M, \mu)}|J, M\rangle$.

En esta, $f(M, \mu)$ es una función no lineal de de $M$ que es equivalente a aplicarle una transformación unitaria al estado coherente (Ecuación (1.38)), que es no-lineal 
en el operador colectivo $J_{z}$, esto es $|\theta, \phi, \mu\rangle=U_{s}(\mu)|\theta, \phi\rangle$. Varios modelos simples de este tipo de transformación puede consultarse en el trabajo (Kitagawa and Ueda, 1993). Para entender como se puede comprimir un estado coherente tomemos como ejemplo la transformación unitaria

$$
U_{s}(\mu)=e^{-i \mu J_{z}^{2}}
$$

que es corresponde con el operador de evolución de un modo del campo en presencia de un medio Kerr, cuyo Hamiltoniano de interacción es proporcional a $J_{z}^{2}$. Si se aplica la transformación de la Ecuación (1.63) a los operadores escalera $J_{ \pm}$se generan fases no lineales, esto es

$$
\begin{gathered}
J_{+}(\mu)=U_{s}^{\dagger} J_{+} U_{s}=J_{+} e^{2 i \mu\left(J_{z}+1 / 2\right)}, \\
J_{-}(\mu)=U_{s}^{\dagger} J_{-} U_{s}=e^{-2 i \mu\left(J_{z}+1 / 2\right)} J_{-},
\end{gathered}
$$

que conducen a la redistribución de las fluctuaciones cuánticas en el plano $x-y$. Es simple de ver que como el operador $J_{z}^{2}$ conmuta con $\left\{J_{+}, J_{-}\right\}=J_{+} J_{-}+J_{-} J_{+}$y con $J_{z}$ estas componentes no se verán afectadas por la transformación de la Ecuación (1.63). A este tipo de transformaciones se las llama de "rotación de torsión" Basados en estas ideas, Kitagawa y Ueda (1993) definieron un parámetro de squeezing respecto de la mínima fluctuación de la componente de espín que está en el plano perpendicular al espín medio. Sea la terna ortogonal $\left\{\mathbf{n}, \mathbf{n}_{\perp(\delta)}, \mathbf{n}_{\perp(\delta+\pi / \mathbf{2})}\right\}$ donde $\mathbf{n}$ tiene la dirección del espín medio y $\delta$ mide el ángulo alrededor del vector espín medio en el plano ortogonal respecto de una dirección dada. La componentes del espín en las direcciones perpendiculares a $\mathbf{n}$ se escriben

$$
\begin{aligned}
J_{\delta} & =\left(\mathbf{J} \cdot \mathbf{n}_{\perp(\delta)}\right), \\
J_{\delta+\pi / 2} & =\left(\mathbf{J} \cdot \mathbf{n}_{\perp(\delta+\pi / \mathbf{2})}\right),
\end{aligned}
$$

y las relaciones de conmutación entre estas quedan

$$
\left[J_{\delta}, J_{\delta+\pi / 2}\right]=(\mathbf{J} \cdot \mathbf{n})
$$

Este conmutador permite escribir la siguiente relación de incerteza

$$
\left(\Delta J_{\delta}\right)^{2}\left(\Delta J_{\delta+\pi / 2}\right)^{2} \geq \frac{1}{4}|\langle J\rangle|^{2},
$$


y de esta podemos definir el factor de squeezing como

$$
\xi^{2}=\frac{2\left(\Delta J_{\perp}\right)^{2}}{|\langle J\rangle|}
$$

donde $\left(\Delta J_{\perp}\right)^{2}=\min _{\delta}\left(\left(\Delta J_{\delta}\right)^{2}\right)$ es el mínimo valor que toman las fluctuación en una de las direcciones perpendiculares al vector espín medio. Se dirá que el sistema esta comprimido en incerteza de espín si $\xi^{2}<1$. Esta definición de squeezing tiene dos claras ventajas respecto de haberlo definido en la forma canónica a partir de la Ecuación (1.62). Por un lado indica si el sistema tiene una compresión en las fluctuaciones del espín en una dirección que está sobre el plano perpendicular sobre el espín medio y además si el sistema se encuentra correlacionado. Otra característica que presenta esta definición es que con la elección de esta relación de conmutación el limite cuántico estándar es una constante.

Otra definición posible, y de uso frecuente, del parámetro de squeezing para sistemas de dos niveles es la introducida por Wineland et al. (1994)

$$
\xi^{2}=\frac{N\left(\Delta J_{\perp}\right)^{2}}{|\langle\bar{J}\rangle|^{2}} .
$$

Este parámetro cuantifica la compresión en la incerteza del espín y además la mejora en la precisión de la medida en experimentos espectroscópicos en sistemas de $N$ átomos de dos niveles, debido a que el sistema se encuentra comprimido en incerteza.

\subsection{Modelo de Dicke y Jaynes-Cummings}

En esta sección se introducen los modelos teóricos que describen la interacción entre uno (o varios) átomos con un modo (o múltiples modos) de radiación electromagnética. Primero se discutirá el modelo de Dicke, que es el modelo típico para describir un átomo interactuando con múltiples modos del campo electromagnético. Luego, se introducirá el modelo de Jaynes-Cummings y se centrará la atención en la interacción de un modo de radiación y sistemas de dos niveles. 


\subsubsection{Hamiltoniano de Dicke para un átomo interactuando con radiación}

Consideremos el sistema compuesto de un átomo con un electrón de carga $e$ y masa $m$, en un potencial $V(r)$ en un campo de radiación descripto por el potencial vector $\mathbf{A}(\mathbf{r}, t)$. El Hamiltoniano de dicho sistema se escribe

$$
H=\frac{1}{2 m_{e}}\left(\mathbf{p}-\frac{e}{c} \mathbf{A}\right)^{2}+V(r)+H_{F}=H_{A}+H_{F}+H_{I}
$$

donde

$$
H_{A}=\frac{\mathbf{p}^{2}}{2 m_{e}}+V(r)
$$

representa el Hamiltoniano del átomo libre, $\mathbf{p}$ es el momento del electrón, $\mathbf{r}$ es la posición relativa del electrón al núcleo atómico. El término del Hamiltoniano que describe el campo de radiación libre es (ver Apéndice A)

$$
H_{F}=\sum_{k} \hbar \omega_{k} a_{k}^{\dagger} a
$$

y la parte que describe la interacción átomo-campo electromagnético es

$$
H_{I}=-\frac{e}{2 m_{e} c}(\mathbf{p} \cdot \mathbf{A}+\mathbf{A} \cdot \mathbf{p})+\frac{1}{2 m_{e}}\left(\frac{e}{c} \mathbf{A}\right)^{2} .
$$

El segundo miembro en la ecuación anterior posee un factor $(e / c)^{2}$, y es muy débil si se lo compara con el primero, así el Hamiltoniano de interacción puede ser aproximado por

$$
H_{I}=-\frac{e}{2 m_{e} c}(\mathbf{p} \cdot \mathbf{A}+\mathbf{A} \cdot \mathbf{p})
$$

Debido a que el operador (escrito en la la representación de Schrödinger (ver ApéndiceA, Ecuación (A.14)))

$$
\mathbf{A}(\mathbf{r}, t)=\sum_{k}\left(\frac{2 \pi \hbar c^{2}}{V \omega_{k}}\right)^{1 / 2} \mathbf{e}_{\lambda}\left[a_{k} e^{i \mathbf{k} \cdot \mathbf{r}}+a_{k}^{\dagger} e^{-i \mathbf{k} \cdot \mathbf{r}}\right]
$$

contiene al operador posición $\mathbf{r}$ se tiene que $[\mathbf{A}, \mathbf{p}] \neq 0$. Sin embargo, en el rango de las dimensiones atómicas, la amplitud de $r$ usualmente está restringida al radio de Bohr 5,3 $\times 10^{-11} \mathrm{~m}$ mientras que la longitud de onda visible $k=\frac{2 \pi}{\lambda}=2 \pi \times 10^{6} \mathrm{~m}^{-1}$, por lo cual $\mathbf{k} \cdot \mathbf{r} \approx 3,5 \times 10^{-4} \ll 1$. En este caso se tiene que $e^{i \mathbf{k} \cdot \mathbf{r}} \approx 1$ y $\mathbf{A}(\mathbf{r}) \approx \mathbf{A}(\mathbf{0})$. 
Por lo cual se pueden despreciar la dependencia espacial de los electrones cuando se discute la interacción entre el campo y el átomo. A esta aproximación se la llama aproximación dipolar. En esta aproximación se tiene

$$
[\mathbf{A}, \mathbf{p}]=0
$$

y el Hamiltoniano de interacción entre el átomo y el campo electromagnético se reduce a

$$
H_{I}=-\frac{e}{m_{e} c} \mathbf{A}(\mathbf{0}) \cdot \mathbf{p}
$$

con

$$
\mathbf{A}(0)=\sum_{k}\left(\frac{2 \pi \hbar c^{2}}{V \omega_{k}}\right)^{1 / 2} \mathbf{e}_{\lambda}\left[a_{k}+a_{k}^{\dagger}\right] .
$$

Ahora se discutirá como expresar el momento del electrón p, en término de los operadores de atómicos de espín. La ecuación de autovalores del Hamiltoniano $H_{A}$ en la representación de Schrödinger es

$$
H_{A}|n\rangle=E_{n}|n\rangle
$$

donde $E_{n}$ es el autovalor de $H_{A}$ y $|n\rangle$ es el correspondiente autovector. En la representación de autovectores $\{|n\rangle\}$, el Hamiltoniano puede ser escrito como

$$
H_{A}=\sum_{n} E_{n}|n\rangle\langle n|
$$

Si se introduce el grupo generalizado de operadores atómicos como

$$
\sigma_{n m}=|n\rangle\langle m|,
$$

entonces la Ecuación (1.81) puede ser escrita como

$$
H_{A}=\sum_{n} E_{n} \sigma_{n n}
$$

Cualquier otro operador $G$ que describe el comportamiento atómico puede ser escrito como

$$
G=\sum_{n m}\langle n|G| m\rangle \sigma_{n n}
$$


Los operadores atómicos $\sigma_{n m}$ satisfacen las relaciones de conmutación

$$
\left[\sigma_{i j}, \sigma_{k l}\right]=\delta_{i l} \sigma_{j k}-\delta_{k j} \sigma_{i l}
$$

Para un átomo de dos niveles activos solo se tiene dos autoestados del Hamiltoniano $\{|+\rangle,|-\rangle\}$ (ó $\{|2\rangle,|1\rangle\})$. En este caso, $\sigma_{n m}$ puede ser escrito explícitamente como

$$
\begin{array}{ll}
\sigma_{11}=\left(\begin{array}{ll}
0 & 0 \\
0 & 1
\end{array}\right), & \sigma_{22}=\left(\begin{array}{ll}
1 & 0 \\
0 & 0
\end{array}\right), \\
\sigma_{21}=\left(\begin{array}{ll}
0 & 1 \\
0 & 0
\end{array}\right), & \sigma_{12}=\left(\begin{array}{ll}
0 & 0 \\
1 & 0
\end{array}\right) .
\end{array}
$$

Este conjunto de operadores satisface la relaciones de conmutación de la Ecuación (1.86). Con este conjunto de operadores podemos escribir

$$
\begin{gathered}
I=\sigma_{11}+\sigma_{22}, \\
S_{z}=\frac{1}{2}\left(\sigma_{22}-\sigma_{11}\right), \\
S_{+}=\sigma_{21}, \\
S_{-}=\sigma_{12},
\end{gathered}
$$

que son los operadores de espín atómico que caracterizan al átomo de dos niveles. Estos satisfacen las siguientes relaciones de conmutación

$$
\left[S_{z}, S_{ \pm}\right]= \pm S_{ \pm}, \quad\left[S_{+}, S_{-}\right]=2 S_{z}, \quad\left[S_{+}, S_{-}\right]_{+}=S_{+} S_{-}+S_{-} S_{+}=I .
$$

Utilizando la Ecuación (1.84) y las definiciones (1.88), el Hamiltoniano de un átomo de dos niveles se escribe como

$$
H_{A}=E_{+} \sigma_{22}+E_{-} \sigma_{11}=\frac{\hbar \omega_{0}}{2}\left(\sigma_{22}-\sigma_{11}\right)=\hbar \omega_{0} S_{z}
$$

donde se han elegido

$$
E_{+}+E_{-}=0, \quad \hbar \omega_{0}=E_{+}-E_{-} .
$$

Utilizando la definición Ecuación (1.85), el operador $\mathbf{p}$ para un átomo de dos niveles puede ser escrito como

$$
\mathbf{p}=\langle+|\mathbf{p}|-\rangle S_{+}+\langle-|\mathbf{p}|+\rangle S_{-} .
$$


Teniendo en cuenta que el operador $\mathbf{r}$ cumple

$$
\frac{d}{d t} \mathbf{r}=\frac{1}{i \hbar}\left[\mathbf{r}, H_{A}\right]=\frac{\mathbf{p}}{m_{e}}
$$

se tiene

$$
\begin{aligned}
\langle+|\mathbf{p}|-\rangle & =\frac{i m_{e}}{\hbar}\left(E_{+}-E_{-}\right)\langle+|\mathbf{r}|-\rangle=\frac{i m_{e} \omega_{0}}{\hbar e}\langle+|e \mathbf{r}|-\rangle, \\
\langle-|\mathbf{p}|+\rangle & =-\frac{i m_{e} \omega_{0}}{\hbar e}\langle-|e \mathbf{r}|+\rangle .
\end{aligned}
$$

Es claro que $\langle+|e \mathbf{r}|-\rangle$ es el elemento de matriz del momento dipolar, que en general es un vector complejo. Por lo tanto podemos definir

$$
\langle+|e \mathbf{r}|-\rangle=i \mathbf{D}
$$

siendo D un vector real. Si en la Ecuación (1.92) reemplazamos los resultados de las Ecuaciones (1.93-1.95) queda expresado el operador atómico p en términos de los operadores de espín y el momento dipolar del átomo

$$
\mathbf{p}=-\frac{m_{e} \omega_{0}}{e} \mathbf{D}\left(S_{+}+S_{-}\right) \text {. }
$$

Reemplazando en la expresión del Hamiltoniano de interacción (Ecuación (1.79)), los resultados de las Ecuaciones (1.80 y 1.96) se obtiene

$$
\begin{aligned}
H_{I} & =-\frac{e}{m_{e}}\left(\sum_{k}\left(\frac{2 \pi \hbar c^{2}}{V \omega_{k}}\right)^{1 / 2} \mathbf{e}_{\lambda}\left(a_{k}+a_{k}^{\dagger}\right)\right) \cdot\left(\left(-\frac{m_{e} \omega_{0}}{e}\right) \mathbf{D}\left(S_{+}+S_{-}\right)\right)(1.9) \\
& =\sum_{k} e_{k}\left(a_{k}+a_{k}^{\dagger}\right)\left(S_{+}+S_{-}\right)
\end{aligned}
$$

donde se definieron las constantes de interacción átomo-campo de radiación como

$$
e_{k}=\left(\frac{2 \pi \hbar}{V \omega_{k}}\right)^{1 / 2} \omega_{0}\left(\mathbf{e}_{\lambda} \cdot \mathbf{D}\right) .
$$

Luego, el Hamiltoniano total de un átomo en interacción con el campo de radiación queda

$$
H=\sum_{k} \omega_{k} a_{k}^{\dagger} a_{k}+\hbar \omega_{0} S_{z}+\sum_{k} e_{k}\left(a_{k}+a_{k}^{\dagger}\right)\left(S_{+}+S_{-}\right)
$$


Esta expresión muestra que la energía del campo de radiación esta dada por la superposición infinita de los modos de los fotones con vector de onda k y la energía del átomo está dada por la componente z del operador de espín. El Hamiltoniano de interacción átomo-campo resulta finalmente

$$
V=\sum_{k} e_{k}\left(a_{k}^{\dagger} S_{-}+a_{k} S_{+}+a_{k}^{\dagger} S_{+}+a_{k} S_{-}\right)
$$

El primer término corresponde al proceso en el cual el átomo pasa del estado excitado $|+\rangle$ al estado fundamental $|-\rangle$ y radia un fotón simultáneamente. Inversamente el segundo término describe un proceso en el cual se absorbe un fotón y el átomo se excita pasando del estado $|-\rangle$ al $|+\rangle$. El tercer término representa la excitación atómica con emisión de un fotón, y el cuarto al proceso de desexcitación con la absorción de un fotón. El tercer y cuarto término representan procesos en los cuales no se conserva la energía. Cuando estos términos se desprecian se obtiene la aproximación de onda rotante, el Hamiltoniano de esta se escribe

$$
H_{R W A}=\hbar \omega_{0} S_{z}+\sum_{k} \omega_{k} a_{k}^{\dagger} a_{k}+\sum_{k} e_{k}\left(a_{k} S_{+}+a_{k}^{\dagger} S_{-}\right)
$$

\subsubsection{Modelo de Jaynes-Cummings}

En esta sección se introduce el modelo propuesto por Jaynes y Cummings (1963). Este modelo considera un átomo de dos niveles en un campo electromagnético que presenta un solo modo. Este modelo describe en forma ideal al acoplamiento entre el átomo y el campo electromagnético. Debido a que este modelo puede ser resuelto en forma exacta, en la aproximación de onda rotante, es relevante no solo en óptica cuántica sino también en la física del láser, resonancia magnética nuclear y teoría cuántica de campos.

En este caso, que el campo electromagnético presenta un solo modo, podemos escribir su potencial vector como

$$
\mathbf{A}(0)=\left(\frac{2 \pi \hbar c^{2}}{V \omega}\right)^{1 / 2} \mathbf{e}_{\lambda}\left[a+a^{\dagger}\right]
$$


Si se repiten las cuentas en forma análoga a la que se hizo para el modelo de Dicke se encuentra que el Hamiltoniano de Jaynes-Cummings resulta

$$
H=\omega a^{\dagger} a+\hbar \omega_{0} S_{z}+\frac{\hbar \Omega}{2}\left(a^{\dagger} S_{-}+a S_{+}+a^{\dagger} S_{+}+a S_{-}\right),
$$

donde $\Omega=2 \omega_{0}\left(\frac{2 \pi}{\hbar V \omega}\right)^{1 / 2}\left(\mathbf{e}_{\lambda} \cdot \mathbf{D}\right)$ es la constante de interacción. Si reescribimos este Hamiltoniano en la representación de interacción se obtiene

$$
H_{I}(t)=\frac{\hbar \Omega}{2}\left(a^{\dagger} S_{-} e^{-i\left(\omega_{0}-\omega\right) t}+a S_{+} e^{i\left(\omega_{0}-\omega\right) t}+a^{\dagger} S_{+} e^{i\left(\omega_{0}+\omega\right) t}+a S_{-} e^{-i\left(\omega_{0}+\omega\right) t}\right)(1
$$

Otra forma de ver la validez de la aproximación de onda rotante es estudiar la dependencia temporal de los términos que no conservan la energía. Como se puede ver en el caso de resonancia $\omega_{0} \approx \omega$ se tiene que los dos últimos términos oscilan rápidamente (comparados con los dos primeros) y las contribuciones de estos promediados en el tiempo serán despreciables. Desechando estos términos se obtiene el Hamiltoniano del modelo de Jaynes-Cummings en la aproximación de onda rotante se escribe

$$
H_{R W A}=\omega a^{\dagger} a+\hbar \omega_{0} S_{z}+\frac{\hbar \Omega}{2}\left(a^{\dagger} S_{-}+a S_{+}\right)
$$

\subsection{Medidas cuánticas de no-demolición (MCND)}

En muchos sistemas cuánticos, el proceso de medida de un observable introduce ruido, lo que hace que medidas sucesivas del mismo observable conduzca a diferentes resultados. El ejemplo más simple de un sistema cuántico es el de la partícula libre. $\mathrm{Su}$ Hamiltoniano es

$$
H=\frac{p^{2}}{2 m}
$$

donde $p$ es el operador momento y $m$ la masa de la partícula. El operador posición $x$ puede ser medido con precisión arbitraria en una medición instantánea. Sin embargo, de acuerdo al las relaciones de incertidumbre de Heisenberg para las variables $x$ y $p$, una medida inicial precisa en $x$ perturba fuertemente a $p$ por lo cual

$$
\Delta p \geq \frac{\hbar}{2 \Delta x}
$$


Durante la posterior evolución libre, $p$ introduce cambios en $x$ de acuerdo con

$$
\dot{x}=\frac{1}{i \hbar}[x, H]=\frac{p}{m},
$$

o, en forma equivalente

$$
x(t)=x(0)+\frac{p(0) t}{m} .
$$

Como resultado de esto

$$
\begin{aligned}
{[\Delta x(t)]^{2} } & =[\Delta x(0)]^{2}+\left[\frac{\Delta p(0)}{m}\right]^{2} t^{2} \\
& \geq[\Delta x(0)]^{2}+\left[\frac{\hbar}{2 m \Delta x(0)}\right]^{2} t^{2},
\end{aligned}
$$

lo que muestra, que la siguiente medición se ve deteriorada.

Por este motivo es deseable considerar esquemas de medida que eviten la "acción de retroceso" sobre la variable que se mide. A este tipo de medidas, en las cuales uno monitorea un observable que puede ser repetidamente medido, siendo el resultado de cada medida determinado por el resultado de la medida inicial, se las llama medidas cuánticas de no demolición. Dicho de otra forma, las medidas cuánticas de no demolición requieren que el acto de medida en si mismo no degrade la predecibilidad en medidas posteriores de un observable.

Como se ejemplificará en la Sección 1.6, la utilización de este tipo de medidas, con la elección de una interacción adecuada, no solo permite monitorear un observable de espín sin introducir incertezas en medidas posteriores, sino que además generan la compresión en incertezas en la variable que se observa (Takeuchi et al., 2005). En general una medida cuántica de no demolición, en una señal observable $A_{s}$ de un sistema cuántico $\mathrm{S}$, se mide por la detección de un cambio en el observable $A_{p}$ de un sistema de prueba $P$ acoplado a $S$ durante el tiempo de medida $T$, sin perturbar la subsecuente evolución de $A_{s}$. Podemos entonces tener una secuencia precisa de mediciones de $A_{s}$ y los resultados obtenidos en cada medición serán completamente predecibles por las mediciones previas.

El Hamiltoniano total para un sistema $S-P$ se puede escribir

$$
H=H_{s}+H_{p}+H_{I}
$$


donde $H_{s}, H_{p}$ y $H_{I}$ son los Hamiltonianos: del sistema, del sistema de prueba y de la interacción entre ellos, respectivamente. La ecuación de movimiento para los operadores $A_{s}$ y $A_{p}$ se expresan como

$$
\begin{aligned}
& i \hbar \dot{A}_{s}=\left[A_{s}, H_{s}+H_{I}\right], \\
& i \hbar \dot{A}_{p}=\left[A_{s}, H_{p}+H_{I}\right] .
\end{aligned}
$$

Para que una medida cuántica sea de no demolición las relaciones entre el observable a ser medido $A_{s}$, la lectura de prueba $A_{p}$ y el Hamiltoniano de interacción tiene que satisfacer:

a) Debido a que se mide $A_{s}, H_{I}$ tiene que ser función de $A_{s}$, esto es

$$
\frac{\partial H_{I}}{\partial A_{s}} \neq 0
$$

b) Como la prueba $P$ se utiliza para medir $A_{s}$, esta no puede ser una constante de movimiento $\dot{A}_{p} \neq 0$. El conmutador de $A_{p}$ y $H_{I}$ debe ser por lo tanto distinto de cero

$$
\left[A_{p}, H_{I}\right] \neq 0
$$

c) El observable $A_{s}$ no debe ser ser afectado el acoplamiento a $A_{p}$ durante la medición, lo que implica

$$
\left[A_{s}, H_{I}\right]=0
$$

d) El Hamiltoniano sin perturbar $H_{s}$ no es función de $A_{s}^{c}$, el observable conjugado de $A_{s}$, esto es

$$
\frac{\partial H_{s}}{\partial A_{s}^{c}}=0
$$

Estas condiciones engloban una medida cuántica de no demolición del observable $A_{s}$, que no se vuelve impredecible dada la relación de incerteza impuesta por su variable conjugada $A_{s}^{c}$ por la medición de $A_{s}$, y que la medida de $A_{s}$ no afecta a $A_{s}$ en sí misma, esto es

$$
\left[A_{s}(t), A_{s}\left(t^{\prime}\right)\right]=0
$$




\subsection{Sistemas de interés}

La propuesta de utilizar estados comprimidos de espín para mejorar la precisión en mediciones cuánticas de no demolición fue inicialmente hecha por Kuzmich et al. (1998). Takahashi et al. (1999) propuso una forma simple de realizar este tipo de medidas en átomos fríos utilizando la rotación paramagnética de Faraday y muestra la mejora que presentan las medidas cuánticas de no demolición cuando se realizan sobre estados comprimidos de espín. Varios experimentos han tenido éxito en generar estados de espín comprimidos en incerteza mediante medidas cuánticas de no demolición (Kuzmich et al., 2000; Schleier-Smith et al., 2010b; Takano et al., 2009). Sin embargo el control de dichos estados para realizar rotaciones en la esfera de Bloch en forma robusta es un problema que actualmente está en discusión. En 2004 Geremia et al. reportó la generación y control de estados comprimidos de espín pero posteriormente se retractaron. Recientemente en los trabajos (Takano et al., 2010; Leroux et al., 2010a; Schleier-Smith et al., 2010b; Schleier-Smith et al., 2010a; Leroux et al., 2010b) se reporta la manipulación con éxito de estados comprimidos de espín en este tipo de dispositivos.

A continuación se describen algunos experimentos y propuestas experimentales donde la compresión en incerteza del espín es de interés. En particular se presentará como implementar las medidas cuánticas de no demolición en sistemas de espines atómicos, como los son las trampas magneto ópticas y las cavidades electromagnéticas, y como este esquema de medición puede ser utilizado para generar compresión en la incerteza del espín. Además se comentaran distintos propuestas experimentales en sistemas de átomos fríos, como son las trampas de iones y las redes ópticas que actualmente son de interés.

\subsubsection{Formulación de una MCND en sistemas de espines}

Si una variable de espín $S_{z}$ se la considera una variable cuántica de no demolición debe satisfacer

$$
\left[S_{z}\left(t_{1}\right), S_{z}\left(t_{2}\right)\right]=0,
$$


en ausencia de interacciones con el sistema de prueba, donde $t_{1}$ y $t_{2}$ son tiempos arbitrarios. Si se toma como Hamiltoniano del sistema de espín libre

$$
H_{s}=\omega_{s} S_{z}
$$

con $\omega_{s}$ real, se satisface esa condición. Para que la la interacción pueda ser considerada como de no demolición debe satisfacer

$$
\left[S_{z}, H_{\text {int }}\right]=0
$$

para evitar la acción de retroceso del sistema (condición de la Ecuación (1.117)). Debido a que la variable que se observa, digamos $J_{x}$, debe ser perturbada por la interacción tendrá que satisfacer

$$
\left[J_{x}, H_{\text {int }}\right] \neq 0
$$

que es la condición de la Ecuación (1.117). Con estos puntos en mente se puede adoptar como interacción el Hamiltoniano de la forma

$$
H_{\text {int }}=\alpha S_{z} J_{z}
$$

A continuación se muestra como implementar este tipo de medidas en un sistema atómico.

\subsubsection{Squeezing en trampas magneto ópticas}

Un esquema físico que permite la realización de medidas cuánticas de no demolición sobre un sistema real de espines puede ser llevando a cabo por la medida de la rotación paramagnética de Faraday de luz de prueba fuera de resonancia. La Figura1.7 muestra un esquema en el que se pueden realizar medidas cuánticas de no demolición en un sistema de espines atómicos.

Se consideran, por simplicidad, un conjunto de átomos que posea un estado fundamental doblemente degenerado y estados excitados óptimamente activos. El estado fundamental del átomo se lo considerará como un sistema de espín $s=1 / 2$ y el 
espín del $i$-ésimo átomo se lo escribe $\mathbf{s}_{i}$. Con esa notación el operador colectivo de espín se escribe $\mathbf{S}=\sum_{i} \mathbf{s}_{i}$.

Se utiliza un haz láser fuera de resonancia, para medir una de las componentes de espín $(\mathbf{S})$, digamos, $S_{z}=\sum_{i} s_{i z}$. Si se expresa el láser de prueba que se propaga como

$$
E(t)=i \omega\left(a_{+} \mathbf{e}_{+}+a_{-} \mathbf{e}_{-}\right) e^{i k z-\omega t}+c . h .
$$

donde $a_{ \pm}$y el vector unitario $\mathbf{e}_{ \pm}$expresan el operador de aniquilación y el vector de polarización para fotones polarizados $\sigma_{ \pm}$respectivamente. Donde $k$ es le vector de onda y $\omega$ es la frecuencia del láser. Utilizando la representación de Schwinger a partir de $a_{ \pm}$se pueden construir los operadores de $J_{x}, J_{y}, J_{z}$ y $N_{J}$ como

$$
\begin{array}{r}
J_{x}=\left(a_{+}^{\dagger} a_{-}+a_{-}^{\dagger} a_{+}\right) / 2 \\
J_{y}=\left(a_{+}^{\dagger} a_{-}-a_{-}^{\dagger} a_{+}\right) / 2 i \\
J_{z}=\left(a_{+}^{\dagger} a_{+}+a_{-}^{\dagger} a_{-}\right) / 2 \\
N_{J}=J_{=}\left(a_{+}^{\dagger} a_{+}+a_{-}^{\dagger} a_{-}\right) / 2
\end{array}
$$

Es simple comprobar que $\left[N_{J}, \mathbf{J}\right]=0$ y que $\mathbf{J}^{2}=N_{J}\left(N_{J}+1\right)$. El operador $\mathbf{J}$ junto con $N_{j}$ se pueden considerar como la versión cuántica de la parametrización de Stokes de un estado de polarización.

Si la intensidad del láser fuera de resonancia es lo suficientemente débil se puede mostrar (Takahashi et al., 1999) que la interacción entre el campo electromagnético y los niveles atómicos es de la forma del Hamiltoniano de la Ecuación (1.125). Las medidas de no demolición pueden realizarse mediante el dispositivo de la Figura 1.7. Los átomos fríos se mantienen mediante una trampa magneto-óptica (Apéndice C.4). Luz polarizada incide sobre los átomos y es rotada en función de la interacción con los átomos. Luego de pasar por un divisor de haz y los fotodetectores miden una señal que es proporcional al número de fotones polarizados en $x$ e $y$ que llegan a cada uno. La diferencia entre el número de fotones es proporcional a $J_{x}$ ya que.

$$
a_{x}^{\dagger} a_{x}-a_{y}^{\dagger} a_{y}=a_{+}^{\dagger} a_{-}+a_{-}^{\dagger} a_{+}
$$

Takashi et al (1999) muestran que el rendimiento de este esquema de medida se 


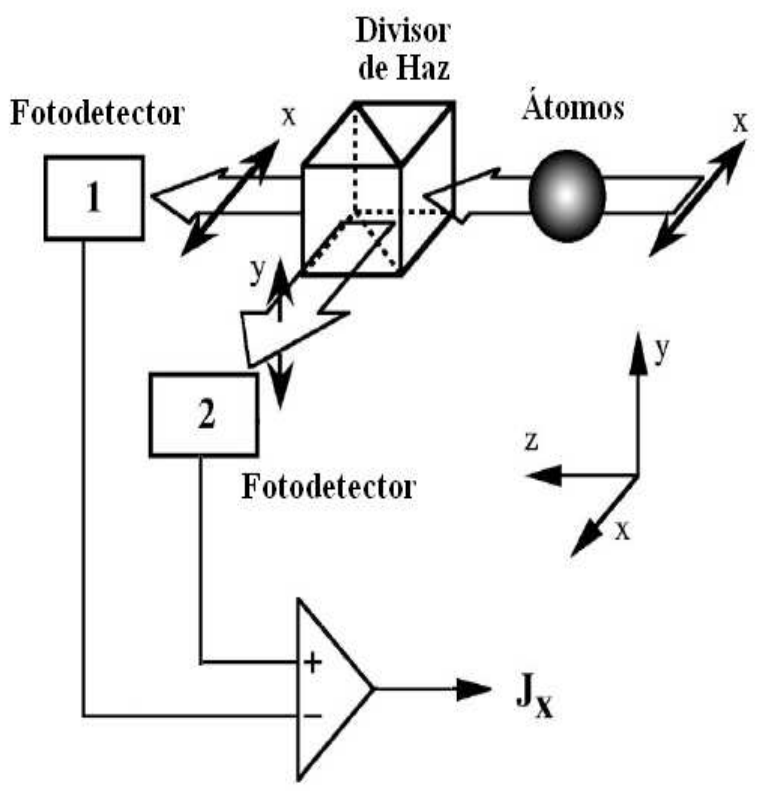

Figura 1.7: Experimento para realizar una medida cuántica de no demolición en un sistema de espines. Luz polarizada fuera de resonancia interactúa débilmente con el sistema de átomos. La rotación paramagnética de Faraday de la polarización puede ser medida utilizando un polarímetro que consiste en un divisor de haz y dos fotodetectores. 
ve favorecido cuando se miden estados comprimidos de espín. Resultados recientes de manipulación de estados comprimidos de espín, y medición de sus componentes mediante esta técnica se reportan en (Takano et al., 2010).

\subsubsection{Cavidades electromagnéticas}

En (2005) Takeuchi te al. propusieron un método para generar compresión en la incerteza del espín de una muestra de átomos fríos. La idea básica fue utilizar átomos en interacción con luz fuera de resonancia de manera que el Hamiltoniano de interacción tome la forma que vimos en la Sección anterior, esto es

$$
H_{\text {int }}=\alpha S_{z} J_{z}
$$

donde $\alpha$ es una constante real, $S_{z}$ es el operador colectivo de la proyección del espín atómico sobre el eje z y $\mathbf{J}$ es la versión cuántica del vector de Stokes y cuyas componentes satisfacen las relaciones de conmutación usuales de momento angular $\left[J_{i}, J_{j}\right]=i \epsilon_{i j k} J_{k}$.

La idea básica es aplicar un pulso de luz cuya componente $J_{z}$ sea proporcional a la componente colectiva de espín atómico $S_{z}$, como un campo magnético ficticio para generar una interacción efectiva $H_{\text {intefec }} \propto S_{z}^{2}$ lo cual produce un efecto de "rotación de torsion" en el sentido expuesto en la Sección 1.3.2 y que se detalla el trabajo (Kitagawa and Ueda, 1993).

Para diseñar esta interacción (Ecuación (1.132)), Takeuchi te al. (2005) propusieron el sistema que se muestra en la Figura. 1.8. Inicialmente un pulso débil de luz $\left|\Psi_{J}\right\rangle$ linealmente polarizado en la dirección del eje $x$, y que contiene $2 J(\gg 1)$ fotones en promedio, se enfoca para que atraviese una muestra de átomos $\left|\Psi_{s}\right\rangle$ que también están polarizados en la dirección del eje $x$ y poseen un espín total $S$. Los valores medios de las componentes de Stokes y de espín colectivo atómico son

$$
\begin{aligned}
\left\langle J_{x}\right\rangle & =J, \\
\left\langle J_{y}\right\rangle=\left\langle J_{z}\right\rangle & =0, \\
\left\langle S_{x}\right\rangle & =S,
\end{aligned}
$$




$$
\left\langle S_{y}\right\rangle=\left\langle S_{z}\right\rangle=0
$$

Debido a que el pulso de luz es un estado coherente, las relaciones de conmutación pueden ser aproximadas como $\left[J_{y}, J_{z}\right]=i J$. El pulso de luz pasa primero a través de los átomos y el plano de polarización es rotado. A esta se la llama "primera interacción" y su tiempo de interacción se etiqueta como $t_{1}$. El vector de Stokes se transforma en

$$
\mathbf{J}^{F I}=e^{i t_{1} H_{i n t}} \mathbf{J} e^{-i t_{1} H_{\text {int }}}
$$

cuya componente $y$ se puede aproximar como

$$
J_{y}^{F I} \simeq J_{y}+\alpha t_{1} J S_{z}
$$

para $\alpha t_{1} J S_{z}$. Debido a que el promedio de $J_{y}^{F I}$ toma el valor

$$
\left\langle\Psi_{j}\left|J_{y}^{F I}\right| \Psi_{j}\right\rangle=\alpha t_{1} J S_{z}
$$

se puede decir que la información de $S_{z}$ se copia y se mantiene en $J_{y}^{F I}$. Hay que tener en cuenta que $\left[S_{z}, H\right]=0$ por lo cual es una constante de movimiento.

Luego el pulso pasa dos veces a través de una placa de $\lambda / 8$ debido a que refleja en un espejo. Como resultado de esto una diferencia de $\lambda / 4$ se introduce entre los dos modos ortogonales de la polarización lineal. A esta operación que se realiza sobre e pulso de luz se lo llama "operación local". El vector de Stokes se escribe como

$$
\mathbf{J}^{L O}=e^{i \pi / 2 J_{x}} \mathbf{J} e^{-i \pi / 2 J_{x}},
$$

y su componente $z$ toma el valor $J_{z}^{L O}=J_{z}^{F I}$. Se puede decir que la información de $S_{z}$ pasa de la componente $J_{z}^{F I}$ a $J_{z}^{L O}$. De esta forma se logra que $J_{z}$ sea aproximadamente proporcional a $S_{z}$. Finalmente el pulso de luz pasa nuevamente a través del conjunto atómico y por lo antes mencionado el Hamiltoniano de interacción será proporcional a $S_{z}^{2}$ lo que produce el efecto de "rotación de torsión" mencionado en la Sección 1.3.2 y que genera compresión en la incerteza del espín atómico.

Recientemente Leroux et. al. en la serie de trabajos (Leroux et al., 2010a; SchleierSmith et al., 2010b; Schleier-Smith et al., 2010a; Leroux et al., 2010b) muestra una 


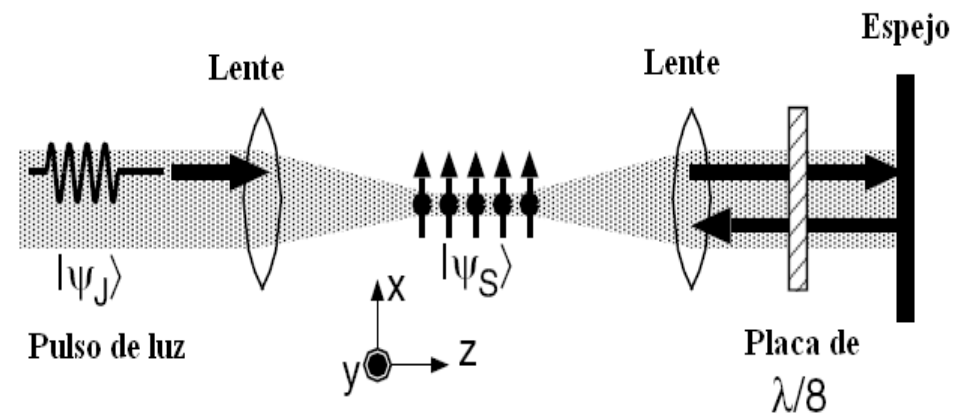

Figura 1.8: a) Un haz de luz linealmente polarizado para a través del conjunto de átomos y el plano de polarización es rotado. El ángulo de rotación es proporcional a $S_{z}$ y se convierte en circularmente polarizada luego de pasar por la placa de $\lambda / 8$ dos veces. Cuando el pulso pasa nuevamente a través de los átomos, induce una rotación no lineal en el conjunto atómico alrededor del eje z como si fuera un campo magnético ficticio, como se describe con mas precisión en el texto.

forma robusta de implementar este método para generar squeezing en una muestra de átomos de ${ }^{87} R b$ y secuencias de control sobre las rotaciones de estados comprimidos de espín en la esfera de Bloch.

\subsubsection{Redes ópticas}

Las redes ópticas son potenciales periódicos que se crean en la intersección de dos (o más) haces de luz coherente que se propagan en direcciones opuestas, uno de otro, e interfieren entre sí. De esta forma, se crea una onda estacionaria con un patrón de interferencia periódico de franjas brillantes y obscuras. La luz induce un momento dipolar eléctrico, en los átomos de un gas ultra frío, modificando su energía. Dependiendo de la frecuencia de la luz los átomos son empujados hacia las franjas brillantes u obscuras confinándolas en pequeñas regiones del espacio. Adicionando lasers que interfieren en distintas direcciones se pueden crear estructuras de potencial de dos y tres dimensiones (Figura 1.9). 
Las redes ópticas proveen una forma de realizar sistemas teóricos simplificados de materia condensada en una forma experimental. Como ejemplo de esto se puede mencionar la utilización de mínimos de potencial en tres dimensiones ocupados por átomos para simular cristales y las simulaciones de transiciones de fase superconductoraislador de Mott (Jaksch et al., 1998; Greiner et al., 2002). De esta forma, sirven para implementar las ideas de Feynman sobre las "simulaciones cuánticas" de un sistema cuántico "complicado" utilizando uno simplificado (Feynman, 1982).

Una propuesta experimental que es de particular interés para esta tesis es la realizada por Sørensen y Mølmer (1999), en la cual mediante el desplazamiento relativo de dos redes ópticas se pueden producir interacciones similares a las ferromagnéticas y antiferromagnéticas en materia condensada.

\subsubsection{Trampas de iones}

Las trampas de iones son dispositivos que confinan partículas cargadas en una región acotada del espacio utilizando campos eléctricos y magnéticos. Estos son dispositivos experimentalmente "limpios" para los cuales la óptica cuántica ofrece técnicas adecuadas para la manipulación y medida de los estados cuánticos (Duan et al., 2003). Dentro de esta familia de dispositivos, los más conocidos para confinar partículas cargadas son la trampa de Penning y la trampa de Paul. La trampa de Penning fue desarrollada por Hans Dehmelt (1967) en la universidad de Washington y la utilizó para realizar medidas espectroscópicas de precisión en iones atrapados y posteriormente, para el estudio de simetrías en física. El funcionamiento básico de este tipo de trampas es el siguiente: se colocan dos placas conductoras paralelas y se las conecta a un cierto potencial, de manera que se carguen con carga del mismo signo que los iones que se desean capturar (Figura 1.10(a)). Las cargas en los planos generan superficies equipotenciales donde los iones se mueven con libertad. Para restringir el movimientos de estos en una región del plano equipotencial se utiliza un campo magnético perpendicular a las superficies de los planos (Figura 1.10(b)). La trampas de Penning modernas utilizan en lugar de un par de placas paralelas, dos hiperboloides de revolución que definen un potencial armónico en el centro de 
la trampa(Figura 1.10(c)).

Actualmente el tipo de trampas de iones más utilizadas, para implementar secuencias de cálculo de computación cuántica, son las trampas lineales de Paul. Esta trampas consisten en cuatro barras conductoras, como se muestran en la Figura 1.10(d). A estas se les aplica una variación sinusoidal de potencial, de algunos MGHz (radiofrecuencia), de manera que dos barras diametralmente opuestas tengan igual carga eléctrica y las otras dos contraria. Para cierta amplitud de voltaje, el promedio temporal del campo oscilante genera un pseudo-potencial armónico que tiene un mínimo en el eje de la trampa. Para restringir el movimiento de los iones en el eje se utilizan dos tapas metálicas que se colocan en los extremos de la trampa y se conectan a un potencial fijo.

La utilización de este tipo de dispositivos como computadoras cuánticas fue propuesto por Ignacio Cirac y Peter Zoller en (1995), y debido a esto, la implementación experimental de estas ideas han tenido un gran desarrollo (Garcia-Ripoll and Cirac, 2003). La idea básica es utilizar estados internos electrónicos de los iones como qbits (espines) y utilizar los grados vibracionales de los iones en el pozo de potencial de la trampa, para crear compuertas cuánticas y crear entrelazamiento (Leibfried et al., 2003; Nielsen and Chuang, 2000). Además de esto, utilizando ideas similares, recientemente se ha mostrado que las trampas de iones pueden ser utilizadas para estudiar una amplia variedad de sistemas cuánticos en interacción (Cirac and Zoller, 1995; Monroe et al., 1995), entre estos en el trabajo de Deng et al.(2005) se muestra como utilizar trampas de iones para simular modelos de Ising. 
a)

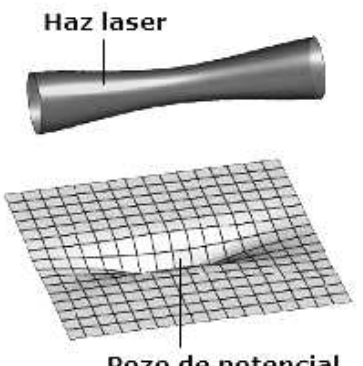

b)

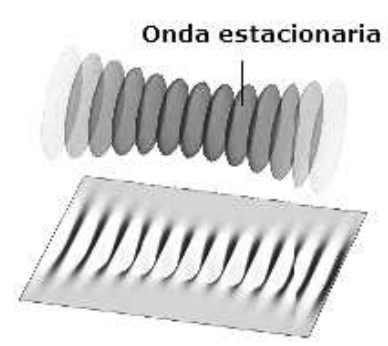

c)

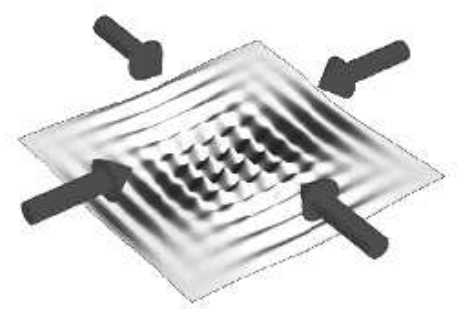

d)
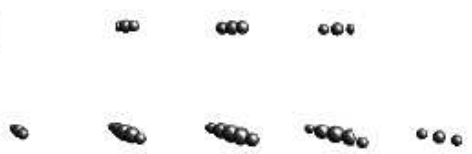

8

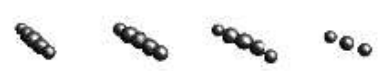

$\&$ \& $\%$

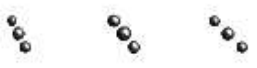

Figura 1.9: La luz láser crea diferentes formas de potenciales ópticos. a) El haz láser crea potenciales atractivos o repulsivos a lo largo de su eje de propagación, que son proporcionales a la intensidad de la luz. b) En la intersección de dos haces láser que se propagan en direcciones contrarias, se puede formar una onda estacionaria sinusoidal. Así, átomos ultra fríos pueden ser atrapados en mínimos de potencial que ocurren cada cuarto de onda y que están separados por una barrera de potencial entre sí. Este es el principio de las redes ópticas unidimensionales. Agregando otro par de haces, en el ángulo correcto, se pueden crear redes bidimensionales (c), y tridimensionales (d). Estas últimas se las llaman redes ópticas "cristalinas" donde los átomos son atrapados en los mínimos de potencial. Las redes ópticas reales tienen millones de sitios y cambiando los ángulos y longitudes de onda de los haces se pueden obtener diferentes geometrías. 

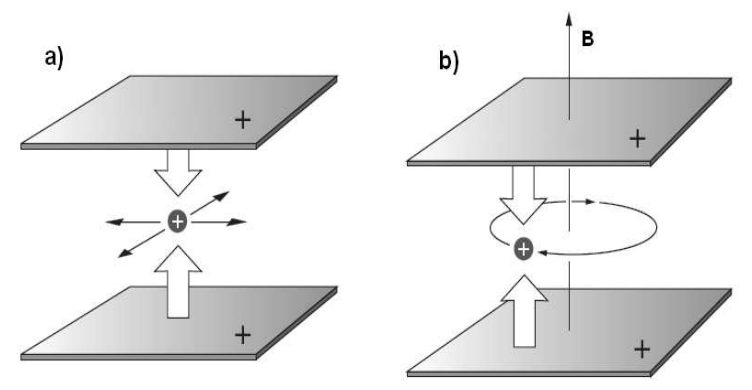

d)

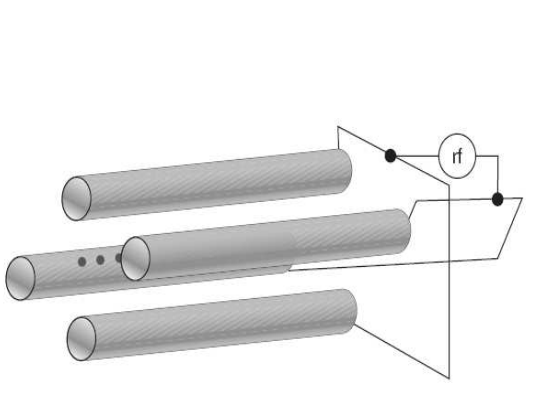

e)
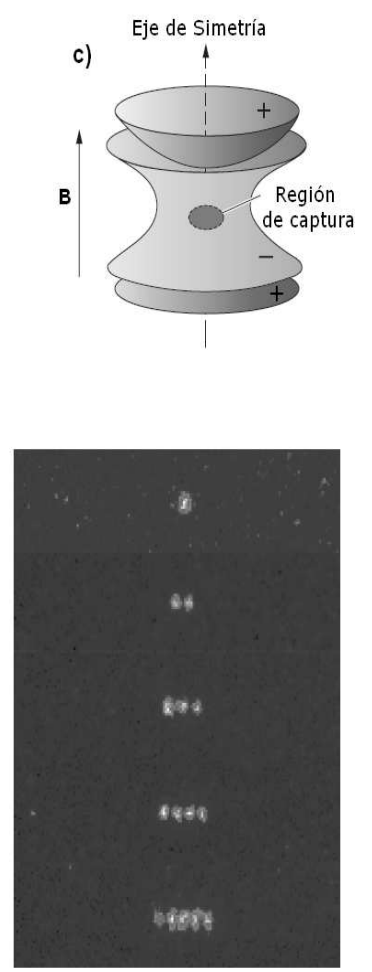

Figura 1.10: a) Un ión interactúa culombianamente con las placas cargadas y su movimiento se confina en el plano paralelo a estas. b) El campo magnético aplicado produce un movimiento circular de los iones (movimiento de ciclotrón) y así se restringe el movimiento en el plano. c)Trampa de Penning de tapas hiperbólicas. Estas producen un potencial armónico en la región de confinamiento. d) Esquema de una trampa de Paul. e) Imagen compuesta, obtenida por fluorescencia, de cadenas de iones de calcio en la trampa de iones del laboratorio de Los Álamos. El espaciamiento entre iones es de 30 $\mathrm{m}$ aproximadamente. (Imágenes tomadas de Holzscheiter(2002)) 


\section{Capítulo 2}

\section{Squeezing en átomos de tres niveles}

En este capítulo se estudia el fenómeno de la compresión de la incerteza del espín (spin squeezing) y del campo electromagnético para un sistema de átomos de tres niveles, en la configuración escalera $(\Xi)$, y que se encuentran en interacción con un modo de radiación. Se introduce el formalismo utilizado para calcular los observables de espín y el factor de squeezing en función del tiempo. En particular se discute la incidencia en el factor de squeezing de: la asimetría en las constante de acoplamiento de la interacción, el tipo de estado inicial en el sector fotónico, y el número de átomos.

\subsection{Introducción}

Las condiciones bajo las cuales el fenómeno de squeezing puede aparecer, desde la perspectiva de la transferencia de información cuántica para átomos y campos de radiación, es objeto de estudio teórico y experimental (Drummond and Ficek, 2004; Echanis and et al., 2005) en la actualidad. En lo que sigue presentamos una reseña de los trabajos más relevantes en el tema. Rangel et al. (2005) estudiaron un sistema consistente en un ion, de dos niveles activos, en una trampa armónica que está localizada en una cavidad electromagnética. Los autores obtuvieron resultados analíticos para el operador densidad total del sistema y muestran la aparición de compresión en incerteza en el movimiento iónico, y en el campo electromagnético. 
También demuestran que existen colapsos y resurgimientos completos y periódicos de los estados de movimiento de los iones y del campo de radiación. Otros autores (Rubin-Linares and Moya-Cessa, 2005) proponen un esquema simple para medir el factor de squeezing y las propiedades de fase de un campo de oscilador armónico al que esta expuesto un átomo. Se muestra que al medir la polarización atómica es posible medir propiedades del campo. Pølsen y Mølmer (2001) muestran la transferencia de correlaciones cuánticas desde los átomos hacia la luz, por medio de la dispersión Raman de un pulso láser intenso sobre una muestra de átomos que presentan compresión en incerteza de espín. En esta referencia también se muestra que bajo condiciones adecuadas, la información cuántica de estados atómicos colectivos puede ser transferida al pulso de luz. El squeezing atómico bajo emisión colectiva fue estudiado por (Yukalov and Yukalova, 2004), en ese trabajo se desarrolla un método para gobernar el comportamiento temporal del factor de squeezing y se estudió la influencia de un vacío efectivo comprimido sobre la emisión colectiva. Zeng et al. (2005) estudian el entrelazamiento y las propiedades de la compresión en incerteza de de espín para tres bosones. Aspectos teóricos y experimentales sobre la compresión en incerteza y entrelazamiento en sistemas de dos niveles se describen en el trabajo de (Josse et al., 2004). El estudio de la compresión en incerteza para un estado no lineal, espín-coherente, se encuentra desarrollado en el trabajo de (Wang, 2001). Estados cuánticos óptimamente comprimidos se encuentran en el trabajo de (Rojo, 2003). La transferencia de espín entre fotones y átomos utilizando el Hamiltoniano de Dicke, se puede encontrar en el trabajo (Dicke, 1954). La compresión de la incerteza atómica en el caso de interacciones átomo-campo considerado en el marco del modelo de Tavis-Cummings (Tavis and Cummings, 1968; Jaynes and Cummings, 1963; Buley and Cummings, 1964; Cummings, 1965) se describe en el trabajo de Genes (2003). En este trabajo se describe el comportamiento de un conjunto de $N$ átomos de dos niveles interactuando con el campo de una cavidad. Otro resultado interesante de este trabajo es el cálculo de la solución analítica en el límite en el cual el número de átomos es grande comparado con el número medio de fotones en un estado coherente en la cavidad. En este límite el grado de la com- 
presión de la incerteza se incrementa al incrementar el número medio de fotones. Una característica común entre los modelos antes mencionados es la interacción entre niveles atómicos y fotones. La estructura de los Hamiltonianos que incluyen este tipo de interacciones pertenecen a la familia de acoplamientos que se puede encontrar en el trabajo de Klein y Marshalek (1991). Estas formas son tratables mediante expansiones bosónicas o mapeos bosónicos (Civitarese and Reboiro, 1998; Civitarese and Reboiro, 1999). Las técnicas de mapeo bosónico permiten la generalización, en formas simples, de las interacciones que se han utilizado hasta ahora, como el Hamiltoniano de Dicke(1954) o el de Tavis y Cummings(1968), por ejemplo. En los trabajos (Wadkiewicz et al., 1987; Ficek and Drummond, 1991a; Ficek and Drummond, 1991b; Javanainen and Gould, 1990) se extendió el esquema adoptado a átomos de tres niveles activos en interacción con un campo de radiación En el trabajo (Javanainen and Gould, 1990) se estudian las poblaciones de átomos de tres niveles interactuando con dos fotones, utilizando técnicas de funciones de correlación.

En este Capítulo de la tesis se describe la dependencia de la compresión en incerteza atómica respecto a la parametrización de las interacciones entre átomos y fotones y respecto a la condición inicial del campo electromagnético. El sistema considerado en esta Capítulo consta de: (a) átomos de tres niveles, y (b) estados fotónicos coherentes para modelar las condiciones iniciales del sistema en el cual la compresión en incerteza puede aparecer. Respecto al punto (a) se presentarán los detalles algebraicos para construir la solución exacta de un Hamiltoniano que describe las excitaciones atómicas de $A$ átomos de tres niveles inducidas por el intercambio de fotones. Relativo al punto (b), se estudió la dependencia de las soluciones con el número medio de fotones en el estado inicial. Los detalles del formalismo se presentan en la siguiente Sección. La ocurrencia de la compresion de las incertezas en un sistema de $A$ átomos de tres niveles en interacción con fotones es numéricamente modelado en la Sección 2.3, donde se presentarán y discutirán las soluciones para diferentes parámetros del modelo y diferentes condiciones iniciales. La evolución del factor de squeezing atómico y fotónico también se muestra en la Sección 2.3. 


\subsection{Formalismo}

El sistema a estudiar consiste en $A$ átomos de tres niveles en interacción con el campo de radiación (Wadkiewicz et al., 1987). Para los operadores de creación (aniquilación) del $i$-ésimo nivel atómico $(i=0,1,2)$, se utiliza $b_{i}^{\dagger}\left(b_{i}\right)$. Los operadores $b_{i}^{\dagger}$ y $b_{i}$ obedecen relaciones de conmutación bosónicas. El Hamiltoniano del sistema es

$$
\begin{aligned}
H= & \omega a^{\dagger} a+\sum_{i} E_{i} S^{i i}+ \\
& g_{1}\left(a S_{+}^{01}+a^{\dagger} S_{-}^{01}\right)+g_{2}\left(a S_{+}^{12}+a^{\dagger} S_{-}^{12}\right),
\end{aligned}
$$

donde $\omega$ es la energía del fotón, $a^{\dagger}(a)$ es el operador de creación (aniquilación) de un fotón, $E_{i}$ es la energía del $i$-ésimo nivel atómico, y $g_{1}$ y $g_{2}$ son las constantes de acoplamiento que describen la absorción (emisión) de un fotón en presencia de una excitación (o desexcitación) atómica de subida (o bajada) entre los niveles 0 y 1 (término proporcional a $g_{1}$ ), y entre los niveles 1 y 2 (término proporcional a $g_{2}$ ). Los operadores $S_{+}^{i j}, S_{-}^{i j}, S_{z_{i \neq j}}^{i j}$ generan la representación simétrica $(N+1)(N+2) / 2$ dimensional del álgebra $S U(3)$. Esto puede ser fácilmente probado escribiendo estos operadores en términos de los operadores de creación (aniquilación), $b_{i}^{\dagger}\left(b_{i}\right)$ bosónicos del $i$-ésimo nivel atómico $(i=0,1,2)$. Así, los operadores

$$
S^{i j}=b_{j}^{\dagger} b_{i}, \quad i, j=0,1,2
$$

satisfacen las relaciones de conmutación

$$
\left[S^{i j}, S^{k m}\right]=\delta_{i m} S^{k j}-\delta_{j k} S^{i m}
$$

Los operadores de inversión atómica

$$
S_{z}^{i j}=\frac{1}{2}\left(S^{j j}-S^{i i}\right)
$$

y los operadores de transición $S_{ \pm}^{i j}$, que satisfacen

$$
S_{+}^{i j}=S^{i j}, \quad S_{-}^{i j}=\left(S_{+}^{i j}\right)^{\dagger}=S^{j i}, \quad i, j=0,1,2(i<j),
$$

son definidos en términos de los operadores de la Ecuación (2.2) y obedecen las mismas relaciones de conmutación. 
La relación de resonancia entre dos fotones (Klimov et al., 1999) es satisfecha si la energía de los niveles atómicos $E_{i}$ cumplen la condición

$$
E_{2}-E_{0}=2 \omega, \quad E_{1}-E_{0}=\omega-\Delta .
$$

\subsubsection{Solución exacta}

El operador

$$
\hat{L}=a^{\dagger} a+2 S_{z}^{02}
$$

conmuta con el Hamiltoniano de la Ecuación (2.1), que por lo tanto, puede ser diagonalizado en la base de estados

$$
\left|n_{b} n_{0} n_{1} n_{2}\right\rangle=\frac{1}{\sqrt{n_{b} ! n_{0} ! n_{1} ! n_{2} !}} a^{\dagger^{n_{b}}} b_{0}^{\dagger^{n_{0}}} b_{1}^{\dagger^{n_{1}}} b_{2}^{\dagger n_{2}}|0\rangle
$$

bajo las condiciones

$$
\begin{gathered}
A=n_{0}+n_{1}+n_{2} \\
N=n_{b}+n_{2}-n_{0},
\end{gathered}
$$

Donde $A$ es el número de átomos, y $N$ es la suma del número de fotones $n_{b}$ mas la diferencia $n_{2}-n_{0}$, entre las poblaciones de los niveles atómicos $i=2$ e $i=0$.

De la diagonalización se obtiene el conjunto de autovalores, $E_{\alpha}$, y autovectores

$$
|\alpha\rangle=\sum_{a \equiv\left\{n_{b}, n_{0}, n_{1}, n_{2}\right\}} c_{\alpha}(a)|a\rangle .
$$

La dimensión del espacio de configuraciones, adoptado para el cálculo está dado por el valor de $N$ a partir del cual se puede garantizar la estabilidad de la función de onda. El procedimiento adoptado se discutirá en la Sección 2.3.

\subsubsection{Evolución temporal}

En la base de los autoestados de $H$, construida como se mencionó anteriormente, la evolución temporal de un dado operador $O$ es expresada como

$$
O(t)=U^{\dagger}(t) O U(t), \quad U(t)=e^{-i H t / \hbar}
$$


El valor esperado en función del tiempo de un operador $O(t)$ se escribe entonces como

$$
\langle O(t)\rangle=\operatorname{Tr}(\rho(t) O)=\sum_{\alpha, \beta}\langle\beta \mid I\rangle\langle I \mid \alpha\rangle\langle\alpha|O| \beta\rangle e^{-i\left(E_{\alpha}-E_{\beta}\right) t / \hbar}
$$

donde se ha definido el operador densidad $\rho(t)=U^{\dagger}(t) \rho(0) U(t)$ a partir de, $\rho(0)=$ $|I\rangle\langle I|$, con $|I\rangle$ es el estado incial del sistema, mientras que $E_{\alpha}$ y $|\alpha\rangle$ son el $\alpha$-ésimo autovalor y autovector del Hamiltoniano respectivamente.

La expresión anterior puede ser escrita en una forma más compacta en términos de las proyecciones del estado inicial $|I\rangle$ y de los autovectores $|\alpha\rangle$, esto es

$$
\begin{array}{r}
\langle O(t)\rangle=\sum_{n, m} T^{*}(n)\langle n|O| m\rangle T(m), \\
T(m)=\sum_{\alpha, n} c_{\alpha, n}^{*} c_{\alpha, m}\langle n \mid I\rangle e^{E_{\alpha} t / \hbar} .
\end{array}
$$

En la Ecuación anterior $|n\rangle$ es una abreviatura de los elementos $\left|n_{b}, n_{0}, n_{1}, n_{2}\right\rangle$ de la base y $c_{\alpha, n} \equiv c_{\alpha}\left(n_{b}, n_{0}, n_{1}, n_{2}\right)$ esto son los coeficientes de los autoestados $|\alpha\rangle$ de la ecuación de autovalores, con autovalor $E_{\alpha}$.

\subsubsection{Parámetro de squeezing en sistemas de tres niveles}

En este caso el objetivo es identificar para qué sistemas el cambio de la población de niveles atómicos pueden ser determinados con exactitud. Es decir, la fluctuación cuántica asociada es lo más pequeña posible. Particularmente es de interés, el estudio de la inversión de población del estado fundamental de los átomos al segundo estado excitado. Como medida de esto, se analizó la evolución del parámetro de squeezing que se define como (Civitarese and Reboiro, 2006; Reboiro, 2008)

$$
Q\left(S_{z}, S_{+}\right)=\frac{2\left(\Delta S_{z}\right)^{2}}{\left|\left\langle S_{+}\right\rangle\right|} .
$$

Donde se utilizó $S_{z}=S_{z}^{02}$ y $S_{+}=S_{+}^{02}$. En este esquema, el squeezing óptimo se obtiene cuando las fluctuaciones de la componente $z$ del espín es mínima. Definiciones similares del espín squeezing se han utilizado en (Prakash and Kumar, 2005). Prakash et al. (2005), discuten la ocurrencia simultánea de squeezing en dos componentes ortogonales de espín, en sistemas de dos niveles. En la próxima Sección se 
presentarán algunos resultados exactos del comportamiento de la compresión de la incerteza de espín en función del tiempo para el sistema modelado por el Hamiltoniano de la Ecuación (2.1).

\subsubsection{Caso de un átomo}

En esta sección se presentan los resultados analíticos para el caso $A=1$. La diagonalización del Hamiltoniano de la ecuación (2.1) se realizó utilizando la base

$$
\begin{aligned}
|a\rangle & =\left|n_{b}, 1,0,0\right\rangle, \\
|b\rangle & =\left|n_{b}-1,0,1,0\right\rangle, \\
|c\rangle & =\left|n_{b}-2,0,0,1\right\rangle,
\end{aligned}
$$

para obtener los autovalores $\lambda_{\alpha}$ y autovectores $\left|\Psi_{\alpha}\right\rangle$, con $\alpha=1,2,3$, que explícitamente tienen las siguientes expresiones:

$$
\begin{aligned}
\lambda_{1}(N)= & \omega\left(n_{b}-1\right), \\
\left|\Psi_{1}(N)\right\rangle= & \mathcal{N}_{1}\left(-g_{2} \sqrt{n_{b}-1}|a\rangle+g_{1} \sqrt{n_{b}}|c\rangle\right), \\
\lambda_{2}(N)= & \omega\left(n_{b}-1\right)-\delta-r\left(n_{b}\right), \\
\left|\Psi_{2}(N)\right\rangle= & \mathcal{N}_{2}\left(g_{1} \sqrt{n_{b}}|a\rangle-\left(r\left(n_{b}\right)+\delta\right)|b\rangle\right. \\
& \left.+g_{2} \sqrt{n_{b}-1}|c\rangle\right), \\
\lambda_{3}(N)=\omega\left(n_{b}-1\right)-\delta+r\left(n_{b}\right), & \\
\left|\Psi_{3}(N)\right\rangle= & \mathcal{N}_{3}\left(g_{1} \sqrt{n_{b}}|a\rangle+\left(r\left(n_{b}\right)-\delta\right)|b\rangle\right. \\
& \left.+g_{2} \sqrt{n_{b}-1}|c\rangle\right),
\end{aligned}
$$

con

$$
\begin{aligned}
\delta & =\frac{\Delta}{2} \\
\mathcal{N}_{1} & =\frac{1}{\sqrt{r\left(n_{b}\right)^{2}-\delta^{2}}}, \\
\mathcal{N}_{2} & =\frac{1}{\sqrt{2}}\left(1-\frac{\delta}{r\left(n_{b}\right)}\right)^{1 / 2} \frac{1}{\sqrt{r\left(n_{b}\right)^{2}-\delta^{2}}}, \\
\mathcal{N}_{3} & =\frac{1}{\sqrt{2}}\left(1+\frac{\delta}{r\left(n_{b}\right)}\right)^{1 / 2} \frac{1}{\sqrt{r\left(n_{b}\right)^{2}-\delta^{2}}},
\end{aligned}
$$




$$
r\left(n_{b}\right)=\sqrt{g_{1}^{2} n_{b}+g_{2}^{2}\left(n_{b}-1\right)+\delta^{2}},
$$

para cada uno de los subespacios etiquetados al fijar el valor de $N=n_{b}+n_{2}-n_{0}$. Para un número variable de fotones en cada subespacio de soluciones etiquetadas por el valor de $N$. El tensor densidad de la Ecuación (2.14) es determinado por la condición inicial $|I\rangle$. Esta puede ser un estado de Fock

$$
|I\rangle=\left|n_{b}, 1,0,0\right\rangle
$$

que representa el producto de los estados de $n_{b}$ fotones por el de un átomo en el estado de menor energía.

Para calcular el parámetro de squeezing atómico se estudió la evolución temporal del operador escalera $S_{+}^{02}$, y de $S_{z}^{02}$ con $N=n_{b}-1$, en este caso se obtuvo

$$
\begin{aligned}
& \left\langle S_{z}\left(n_{b}, t\right)\right\rangle=\left\langle S_{z}^{02}\left(n_{b}, t\right)\right\rangle \\
& =-\frac{1}{2}+\frac{1}{2} \frac{g_{1}^{2} n_{b}}{r\left(n_{b}\right)^{2}} \sin ^{2}\left(r\left(n_{b}\right) t\right) \\
& +\frac{g_{1}^{2} g_{2}^{2} n_{b}\left(n_{b}-1\right)}{\left(r\left(n_{b}\right)^{2}-\delta^{2}\right)^{2}}\left(\left(\cos \left(r\left(n_{b}\right) t\right)-\cos (\delta t)\right)^{2}\right. \\
& \left.\quad+\left(\frac{\delta}{r\left(n_{b}\right)} \sin \left(r\left(n_{b}\right) t\right)-\sin (\delta t)\right)^{2}\right) \\
& \left\langle S_{z}^{2}\left(n_{b}, t\right)\right\rangle=\left\langle\left(S_{z}^{02}\right)^{2}\left(n_{b}, t\right)\right\rangle \\
& =\frac{1}{4}\left(1-\frac{g_{1}^{2} n_{b}}{r\left(n_{b}\right)^{2}} \sin ^{2}\left(r\left(n_{b}\right) t\right)\right) \\
& \left\langle S_{+}(t)\right\rangle=\left\langle S_{+}^{02}\left(n_{b}, t\right)\right\rangle=0 .
\end{aligned}
$$

Por lo tanto, para este caso el fenómeno de squeezing atómico no se observa, sin importar cuantos fotones o niveles atómicos son incluidos.

Para el caso de un estado fotónico coherente (Ecuación (B.14)), con un número medio de fotones $|z|^{2}=n_{b}$, el cálculo conduce a las expresiones

$$
\begin{aligned}
& \left\langle S_{z}(t)\right\rangle=e^{-n_{b}} \sum_{k=0}^{\infty} \frac{n_{b}^{k}}{k !}\left\langle S_{z}(k, t)\right\rangle, \\
& \left\langle S_{z}^{2}(t)\right\rangle=e^{-n_{b}} \sum_{k=0}^{\infty} \frac{n_{b}^{k}}{k !}\left\langle S_{z}^{2}(k, t)\right\rangle, \\
& \left\langle S_{+}(t)\right\rangle=e^{-n_{b}} \sum_{k=0}^{\infty} \frac{n_{b}^{k+1}}{k !} f_{0}(k)(a(k)+i b(k))
\end{aligned}
$$


con

$$
\begin{aligned}
a(n)= & \left(g_{2}^{2}(n-2)+g_{1}^{2}(n-1)\right) f_{1}(n+1) \\
& +g_{1}^{2}(n-1)\left(f_{2}(n-1) f_{2}(n+1)+f_{2}(n-1) f_{2}(n+1)\right), \\
b(n)= & g_{1}^{2}(n-1)\left(f_{1}(n-1) f_{2}(n+1)-f_{2}(n-1) f_{1}(n+1)\right),
\end{aligned}
$$

$\mathrm{y}$

$$
\begin{aligned}
f_{0}(n) & =\frac{g_{1} g_{2} \sqrt{(n+2)(n+1)} e^{2 i \omega t / \hbar}}{\left(g_{1}^{2}(n+2)+g_{2}^{2}(n+1)\right)\left(g_{1}^{2} n+g_{2}^{2}(n-1)\right)} \\
f_{1}(n) & =\cos (\delta t) \cos (r(n) t)+\frac{\delta}{r(n)} \sin (\delta t) \sin (r(n) t)-1 \\
f_{2}(n) & =-\sin (\delta t) \cos (r(n) t)+\frac{\delta}{r(n)} \cos (\delta t) \sin (r(n) t) .
\end{aligned}
$$

Donde las cantidades $f_{0}(k), a(k)$, y $b(k)$ son obtenidas directamente del Hamiltoniano y los autovectores. Hay que notar que, para esta condición inicial, los valores esperados del operador escalera en función del tiempo (2.21) son no nulos. Esto significa que, dependiendo de las constantes de acoplamiento de $H$, el squeezing puede aparecer, esto es $Q\left(S_{z}, S_{+}\right) \leq 1$ (Genes et al., 2003).

\subsection{Resultados y discusión}

En lo que sigue vamos a considerar que la energía de espaciamiento entre niveles atómicos esta fija por la Ecuación (2.6), con $\Delta=0$, así $E_{0}=-\omega, E_{1}=0, E_{2}=\omega$. En todos los casos se han tomado estados coherentes en el sector fotónico del estado inicial. Se considero la parametrización simétrica, $g_{1}=g_{2}$, y una no simétrica, $g_{1} \neq g_{2}$, del Hamiltoniano. El valor medio de fotones del estado coherente se tomó como variable. Se realizaron cálculos para los casos de $A=3, A=6, A=15$ y $A=18$ átomos, respectivamente. En las Figuras (2.1-2.3), se muestran los resultados para la evolución temporal de la inversión atómica, $\left\langle S_{z}(t)\right\rangle$, el factor de squeezing atómico, $Q\left(S_{z}, S_{+}\right)$, y el factor de squeezing del campo $Q(x, p)$ (Genes et al., 2003), que para este caso se empleó la definición canónica (Ecuación (1.57)) con los operadores posición $\left(x=1 / \sqrt{2}\left(a+a^{\dagger}\right)\right)$ y momento $\left(p=-i / \sqrt{2}\left(a+a^{\dagger}\right)\right)$ 
del campo electromagnético. El estado inicial en el sector atómico, corresponde a tomar $n_{0}=A$ (estado fundamental del sistema), mientras que el parámetro $|z|^{2}$ del estado coherente fotónico se fijó con el valor $n_{b}$. Con esta parametrización el Hamiltoniano de la Ecuación (2.1) fue diagonalizado y el tensor densidad (Ecuación (2.14)) se obtuvo para cada subespacio, $A, N$. La Figura 2.1 muestra los resultados para $A=1, g_{1}=g_{2}=1$ y $n_{b}=10$. No se encuentra señal alguna de compresión de incerteza, a pesar de haber utilizado estados coherentes en el sector fotónico. Este resultado puede ser comparado con el que se encuentra en (Genes et al., 2003), donde para el caso de átomos de dos niveles, y estados coherentes para los estados fotónicos, no se obtiene el fenómeno de squeezing atómico. En la Figura 2.2 se muestran los resultados obtenidos para el caso de una parametrización asimétrica, esto es $g_{1}=1, g_{2}=4$. Dicho caso exhibe compresión en incerteza atómica, y el valor central del parámetro de squeezing $\left(Q\left(S_{z}, S_{+}\right)\right)$es del orden de $0,75-0,80$. La evolución temporal de la inversión atómica $\left\langle S_{z}(t)\right\rangle$ es consistente con el valor central $\left\langle S_{z}(t)\right\rangle=-0,4$, mientras que en los resultados de la Figura 2.1 el valor central de $\left\langle S_{z}(t)\right\rangle$ es cero. Los resultados mostrados en la Figura 2.2 señalan una posible dependencia crítica de la compresión en incerteza respecto a la intensidad relativa con que se emiten y absorben fotones en transiciones que involucran el estado base, $n_{0}$, y el estado excitado $n_{2}$, independientemente del valor medio del número de fotones. En la Figura 2.3, se muestra la dependencia del factor de squeezing atómico respecto al número de átomos. Para esto, se tomaron casos con $A=3, A=6, A=15$, y $A=18$, manteniendo fijos $g_{1}=1, g_{2}=6, n_{b}=21$. Se observa que la compresión en incerteza atómica desaparece cuando el número de átomos se incrementa. Estos resultados parecen indicar una fuerte dependencia de la compresión en incerteza atómica con el número de átomos. La comparación entre los resultados que se han obtenido utilizando acoplamientos simétricos y no-simétricos señala la dependencia crítica del squeezing con las constantes relativas de emisión y absorción de fotones en las transiciones entre el estado fundamental $n_{0} \mathrm{y}$ el estado excitado $n_{2}$. Esta es independiente del valor medio de los fotones. Esto es posible visualizarlo en la Figura 2.4 donde se tomaron los mismos parámetros que en la Figura $2.3(A=3, A=6$, 
$A=15$, y $A=18$, manteniendo fijos $g_{1}=1, g_{2}=6$ ), pero utilizando $n_{b}=400$.

\subsection{Conclusiones del capítulo}

En este capítulo se estudió, la aparición de compresión en incerteza en sistemas atómicos de tres niveles en interacción con un campo de radiación. Se encontró que:

a) Utilizando condiciones iniciales consistentes en un número fijo de fotones no conducen observar el fenómeno squeezing, sin embargo, este aparece si se utiliza en el sector fotónico un estado coherente como condición inicial;

b) La transferencia de espín entre átomos y fotones es favorecida si la interacción entre los niveles atómicos y los fotones, es parametrizada en una forma no simétrica. Esto se logra tomando $g_{2}>g_{1}$ en el Hamiltoniano de la Ecuación (2.1) (ya que, el caso $g_{1}>g_{2}$ no condujo a la aparición del mismo). También se encontró que la utilización de un estado coherente no conduce, en forma automática, al la compresión en incerteza, a menos que la interacción considerada sea no-simétrica en la forma explicada con anterioridad;

c) Para una dada parametrización se encuentra que al aumentar el número de átomos se borra la compresión en incerteza. 


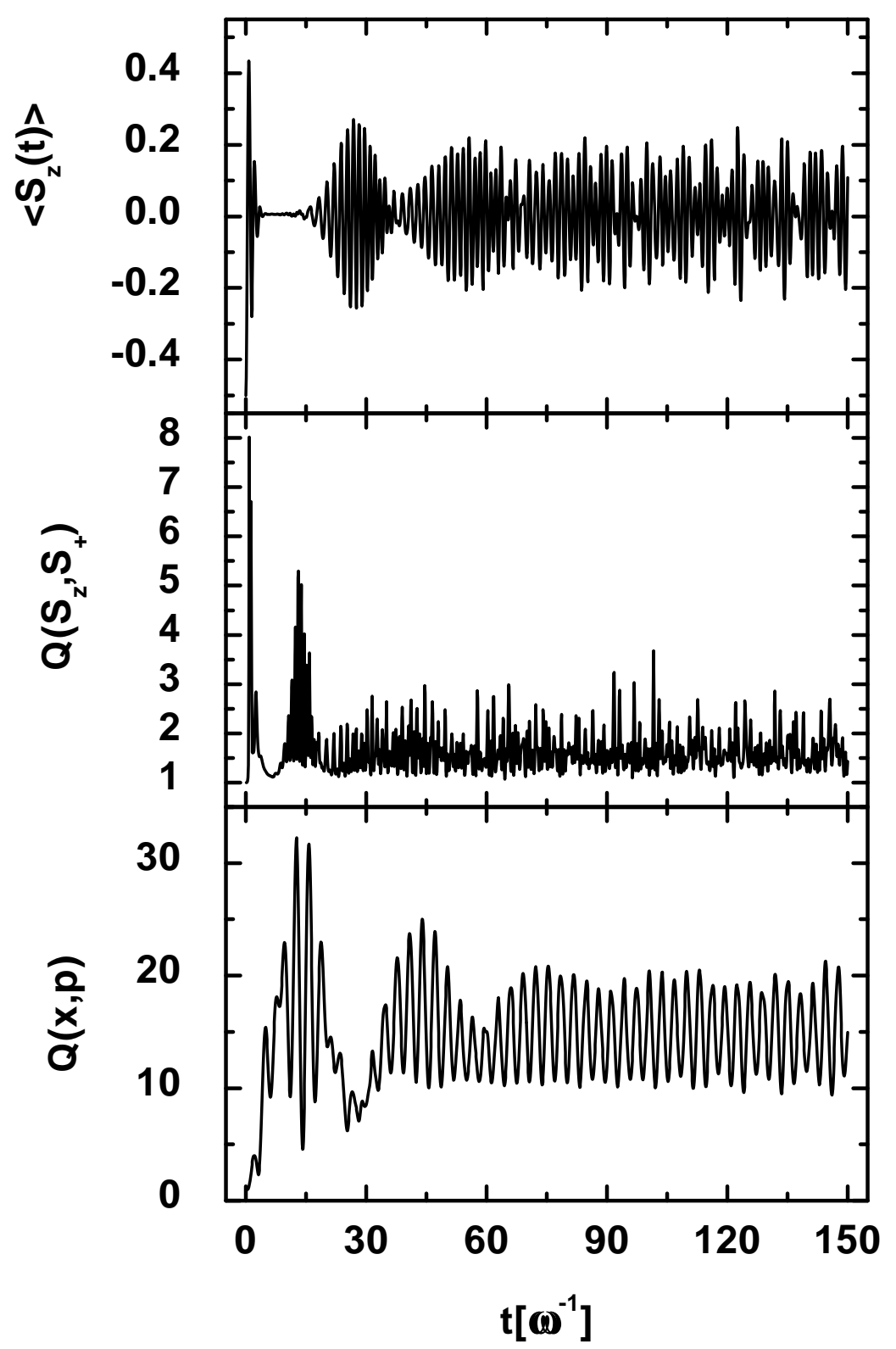

Figura 2.1: Valor medio del operador $\left\langle S_{z}(t)\right\rangle$, factor de squeezing atómico $Q\left(S_{z}, S_{+}\right)$, factor de squeezing del campo electromagnético $Q(x, p)$, en función del tiempo. El sistema consiste en un átomo, inicialmente en el estado fundamental, y un campo de fotones coherente con $n_{b}=10$. Las constantes de acoplamiento se fijaron en $g_{1}=1$ y $g_{2}=1$. 


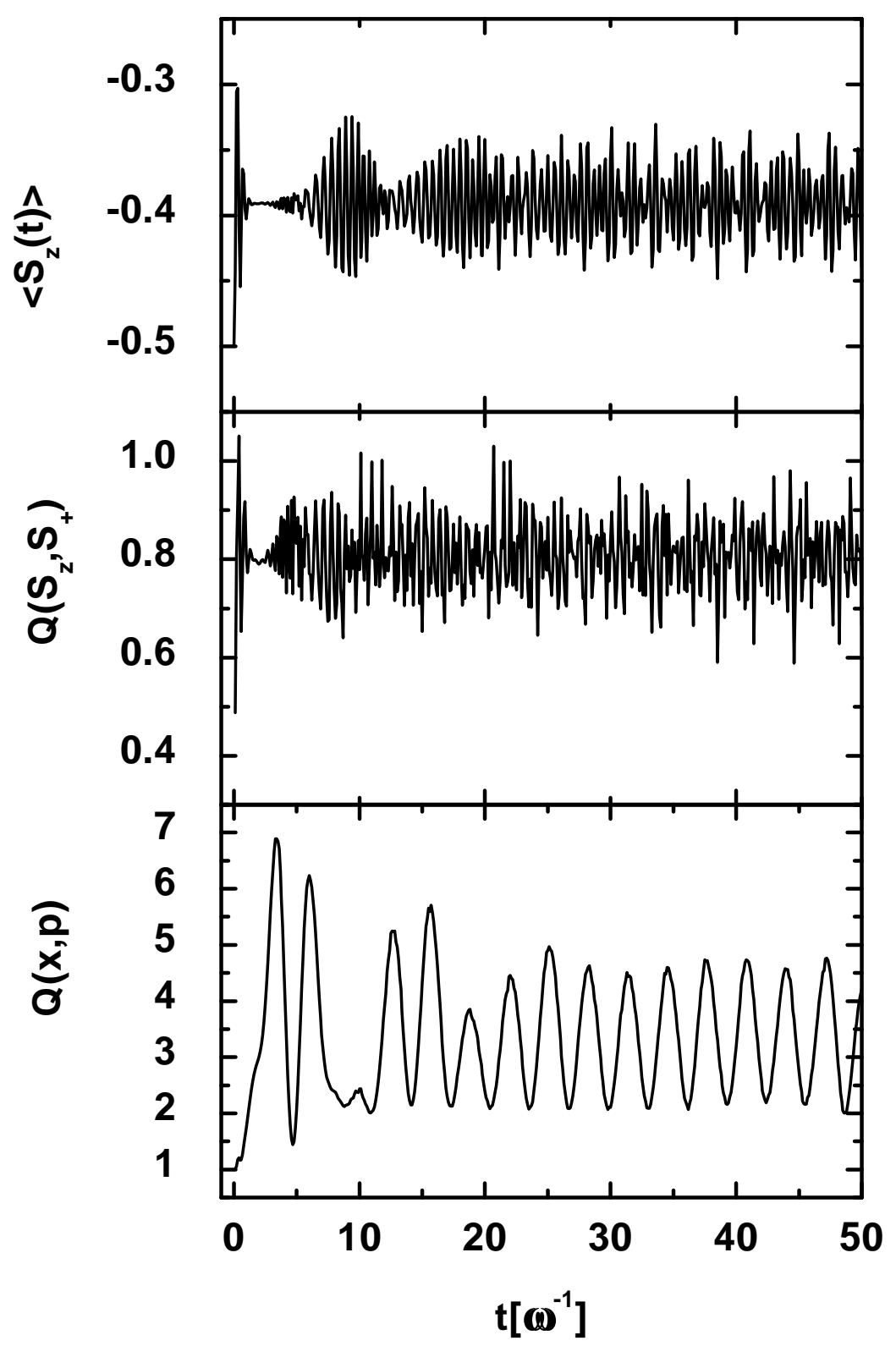

Figura 2.2: Idem. Figura (2.1) con $n_{b}=10, g_{1}=1$, y $g_{2}=4$. 

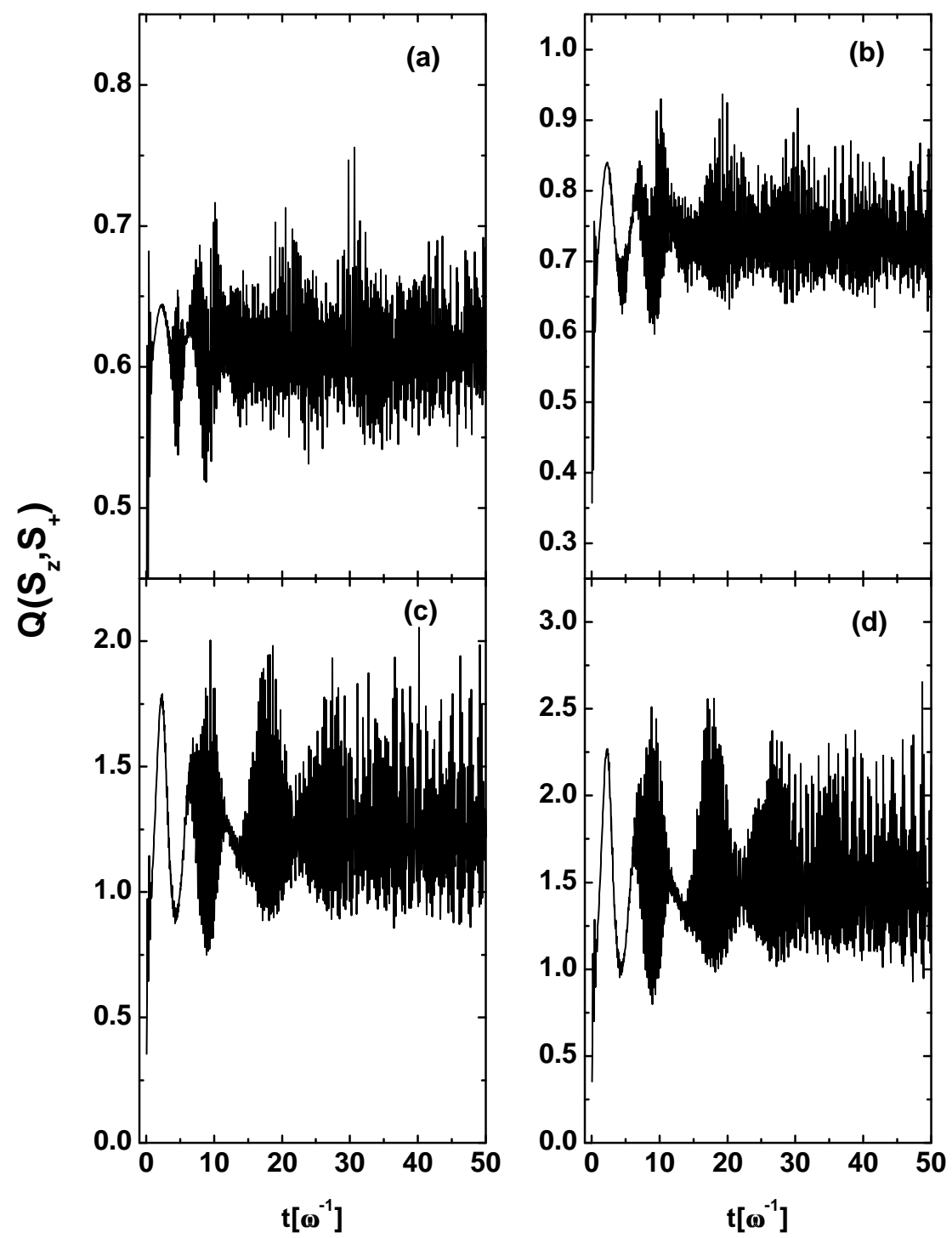

Figura 2.3: Factor de squeezing atómico $Q\left(S_{z}, S_{+}\right)$en función del tiempo. Las constantes de interacción se fijaron en $g_{1}=1$ y $g_{2}=6$. El estado inicial consta de $A$ átomos en el estado fundamental, y el estado coherente con un valor medio de fotones $n_{b}=21$. Los casos (a), (b), (c), y (d), corresponden a sistemas con $A=3, A=6, A=15$, y $A=18$ átomos, respectivamente. 

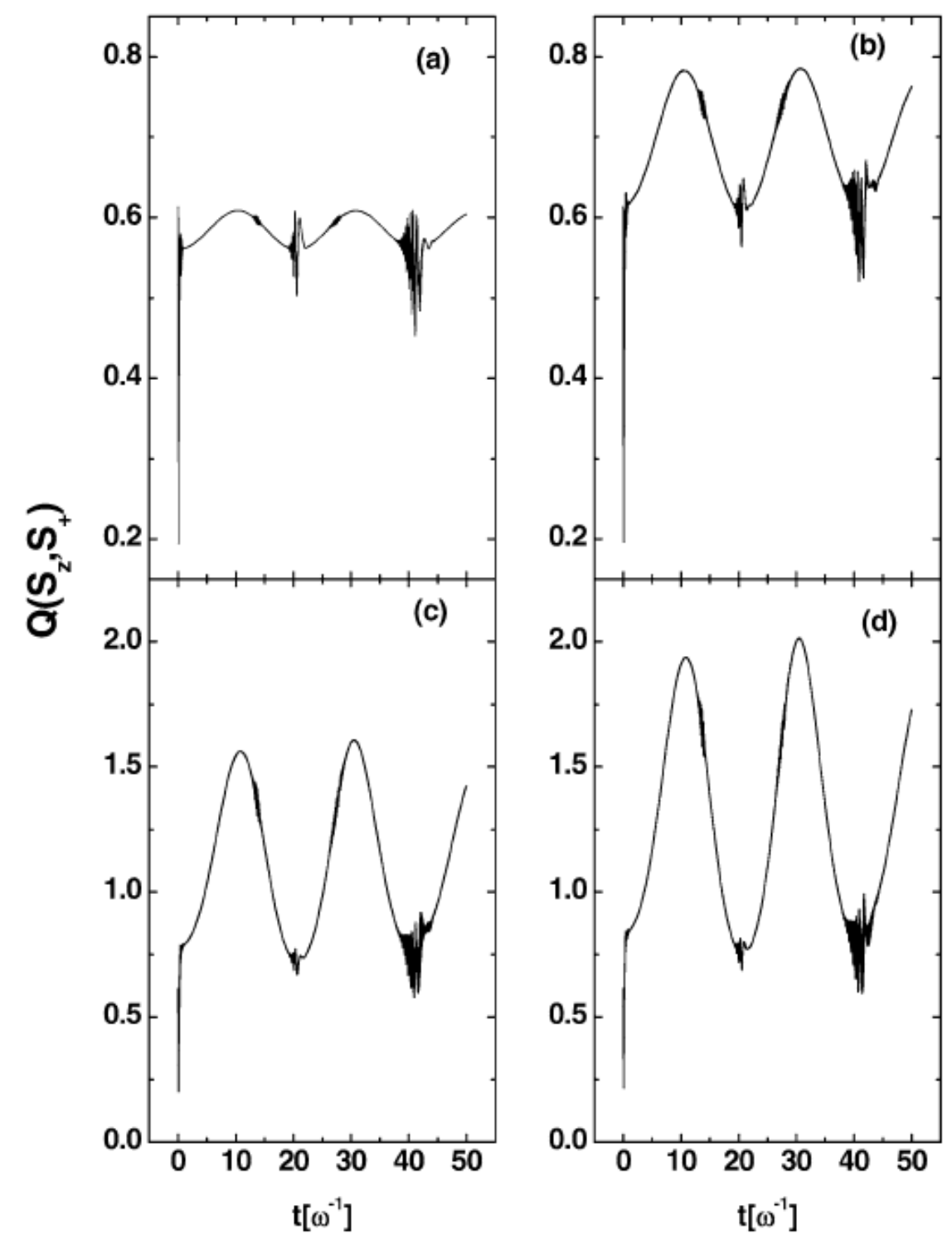

Figura 2.4: Idem. Figura 2.3 con $n_{b}=400$. 


\section{Capítulo 3}

\section{Squeezing en átomos de tres niveles e interacciones dipolares}

En este capítulo se analiza la aparición del fenómeno de squeezing atómico en átomos de tres niveles en una cavidad que presenta dos modos propios de radiación. Se discuten los efectos de incorporar una interacción dipolar efectiva entre átomos. Del estudio realizado resulta que la inclusión de este tipo de interacciones tiende a remover la aparición de la compresión en incerteza atómica, mientras que el incremento en el número medio de los fotones, de un estado coherente, modera este efecto.

\subsection{Introdución}

Recientemente se han publicado diversas propuestas referidas al diseño de "computadoras" cuánticas (Kok et al., 2007; Imai, 2006). Entre estas propuestas figuran, los dispositivos de computación cuántica con átomos neutros (Brennen et al., 1999; Jaksch et al., 1999; Ahn et al., 2000) son particularmente atractivos, debido a los largos tiempos de coherencia de los estados atómicos internos y a las bien desarrolladas técnicas de enfriamiento y captura en redes ópticas, trampas de luz fuera de resonancia, y microtrampas magnéticas (Briegel et al., 2000). En particular, los acoplamientos dipolo-dipolo entre estados de Rydberg proveen una interacción fuerte deseable para la implementación de compuertas de dos qubits en átomos neutros (con los tiempos de operación de la compuerta mucho más cortos que las escalas de tiempo 
asociadas con el movimiento de los átomos en el potencial confinante)(Jaksch et al., 2000; Yavuz, 2006). Un análisis detallado de un dispositivo lógico basado en interacciones dipolo-dipolo de átomos de Rydberg ópicamente atrapados se presentan en (Saffman and Walker, 2005; Saffman and Mølmer, 2008). En la referencia (Lukin et al., 2001), se prestó atención al fenómeno denominado "bloqueo-dipolar", cuando varios átomos están suficientemente cerca, la presencia de un átomo excitado causa un corrimiento en energía de todos los átomos que es lo suficientemente grande como para prevenir excitaciones resonantes de más de un átomo en la muestra (Lukin et al., 2001). El fenómenos de "bloqueo-dipolar" puede usarse para crear conjuntos fuertemente acoplados con un número moderado de átomos (Brion et al., 2007). Johnson et al. (2008) estudiaron oscilaciones de Rabi entre estados fundamentales y de Rydberg en ${ }^{87} R b$. Ellos han observado oscilaciones coherentes de población para un átomo, mientras la presencia de dos o más átomos destruyen la coherencia de la oscilación. La generación de entrelazamiento multipartito es discutido en (Saffman and Mølmer, 2009). Entre los sistemas de estudio son de interés los conjuntos de átomos de tres niveles en presencia de campos láser e interactuando entre sí por medio de interacciones dipolo-dipolo. Por ejemplo se puede ver en las referencias (MacOvei et al., 2005; Kiffner et al., 2007; Wang, 2009) donde se implementa la manipulación coherente de átomos de tres niveles interactuando por medio de interacciones dipolo-dipolo.

En este Capítulo se consideró un sistema de átomos de tres niveles en una cavidad que soporta dos modos (Dantan et al., 2003; Vogel and Blatt, 1992; Dalton et al., 1994; Ficek et al., 1995). Se adoptó un estado coherente para modelar las condiciones iniciales de los campos fotónico y se incluyeron interacciones efectivas dipolo-dipolo entre átomos (Varada and Agarwal, 1992; Agarwal and Patnaik, 2001). La aparición de la compresión en incerteza atómica (Drummond and Ficek, 2004; Cerf et al., 2007) se investigó en base a la asimetría de las constante de acoplamiento en la interacción átomo-fotón del Hamiltoniano propuesto, así como también por la inclusión del término de interacción dipolo-dipolo (Civitarese and Reboiro, 2006; Reboiro, 2008). 
El desarrollo de este Capítulo está esquematizado de la siguiente manera: En la Sección 3.2 se presentaran los detalles del formalismo utilizado; La aparición del fenómeno de squeezing en un sistema de átomos de tres niveles se discute en la Sección 3.3, donde se muestran y discuten los resultados obtenidos; Por último, en la Sección 3.4, se dan las conclusiones.

\subsection{Formalismo}

Se consideró un sistema que consiste en $A$ átomos idénticos de tres niveles en interacción con un campo de radiación (Vogel and Blatt, 1992; Dalton et al., 1994; Ficek et al., 1995; Civitarese and Reboiro, 2006; Reboiro, 2008; Klimov et al., 1999). El Hamiltoniano propuesto es de la forma

$$
H=H_{0, \text { fotón }}+H_{0, a t}+H_{d d}+H_{\text {fotón-at }},
$$

donde

$$
\begin{array}{r}
H_{0, \text { fotón }}=\omega_{a} a^{\dagger} a+\omega_{b} b^{\dagger} b \\
H_{0, a t}=\sum_{i} E_{i} S^{i i}
\end{array}
$$

Para la interaccción dipolo-dipolo efectiva entre átomos hemos adoptado

$$
H_{d d}=g \sum_{\gamma, \gamma^{\prime}}\left(S_{+}^{01}(\gamma)+S_{-}^{12}(\gamma)\right)\left(S_{-}^{01}\left(\gamma^{\prime}\right)+S_{+}^{12}\left(\gamma^{\prime}\right)\right)
$$

En la interacción definida en la Ecuación (3.4) no se consideró el término de autointeracción y se tomaron promedios sobre las posiciones relativas de los átomos, absorbiendo las distancias de interacción en acoplamientos efectivos (Cohen-Tannoudji et al., 1992). Debido a que las configuraciones que estamos tratando incluyen un número pequeño de átomos, esta aproximación no debería afectar mayormente los cálculos, aunque este tipo de aproximación no es válido para arreglos espaciales con un número grande de átomos, donde los efectos de atenuación dependen de la posición de los átomos.

Los átomos interactúan con dos campos láser de frecuencias $\omega_{a} \mathrm{y} \omega_{b}$, respectiva- 


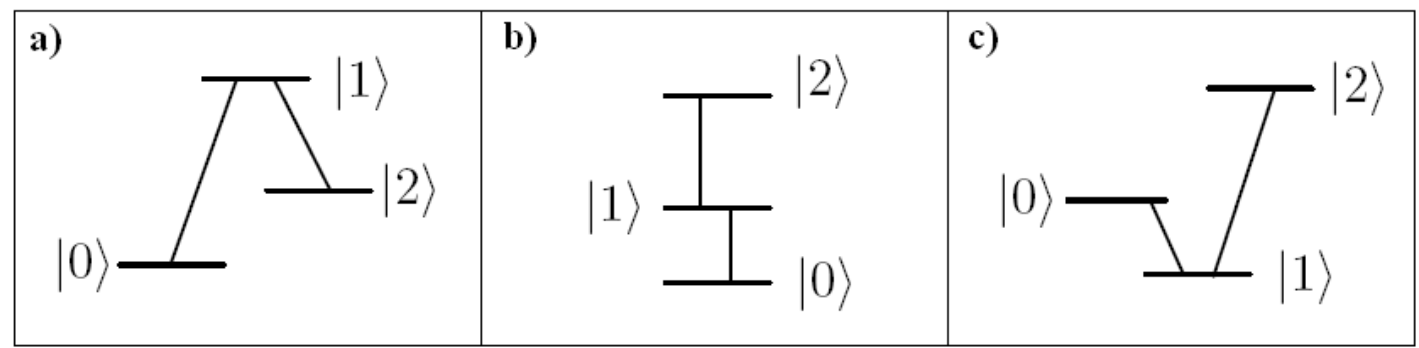

Figura 3.1: Diferentes configuraciones para el término de interacción $H_{\text {fotón-at }}$ Los gráficos (a), (b), y (c) corresponden a las configuraciones $\Lambda, \Xi, \mathrm{y} V$ respectivamente.

mente. Se estudió el comportamiento de los sistemas para las diferentes configuraciones posibles $(\Lambda, \Xi \mathrm{y} V)$ Figura 3.1. Al igual que en el Capítulo anterior se utilizó como interacción fotón-átomo

$$
H_{\text {fotón,at }}=g_{1}\left(a S_{+}^{01}+a^{\dagger} S_{-}^{01}\right)+g_{2}\left(b S_{+}^{12}+b^{\dagger} S_{+}^{12}\right) .
$$

Los operadores $S_{+}^{i j}, S_{-}^{i j}, S_{z_{i \neq j}}^{i j}$ generan la representación simétrica $(N+1)(N+$ 2)/2 dimensional del álgebra $S U(3)$ (ver ecuaciones(2.2)-(2.5)). En la expresión de $H$, de la Ecuación (3.1), $a^{\dagger}(a)$ el operador de creación (aniquilación) de un fotón con energía $\omega_{a}$, mientras que $b^{\dagger}(b)$ corresponden a los modos de energía $\omega_{b}$. $E_{i}$ es la energía del $i$-ésimo nivel atómico. En las Ecuaciones (3.5) y (3.4), $g_{1}$ y $g_{2}$ son las constantes que describen la absorción (emisión) de fotones en presencia de excitaciones atómicas entre los niveles 0 y 1 (término proporcional a $g_{1}$ ), y entre los niveles 1 y 2 (término proporcional a $g_{2}$ ), y g es la constante de acoplamiento efectivo de la interacción dipolar átomo-átomo.

\subsubsection{Solución exacta}

La base de estados del problema, es el producto directo de la base de estados atómicos y la base de estados para los fotones asociados a los modos de radiación. Para la base de estados atómicos se consideró el estado colectivo con $n_{1}$ átomos en el primer estado excitado y $n_{2}$ átomos en el segundo estado excitado $\left(n_{1}+n_{2} \leq A\right)$.

$$
\left|n_{1}, n_{2}\right\rangle=N\left(n_{1}, n_{2}\right) \sum_{p}\left|n_{1}^{p}(1), \ldots, n_{1}^{p}(A), n_{2}^{p}(1), \ldots, n_{2}^{p}(A)\right\rangle
$$




$$
N\left(n_{1}, n_{2}\right)=\left(\left(\begin{array}{c}
A \\
n_{1}
\end{array}\right)\left(\begin{array}{c}
A-n_{1} \\
n_{2}
\end{array}\right)\right)^{-1 / 2},
$$

$\operatorname{con} n_{1}=\sum_{j=1}^{A} n_{1}^{p}(j), n_{2}=\sum_{j=1}^{A} n_{2}^{p}(j)$ y $n_{1}^{p}(j)=n_{2}^{p}(j)=0,1 .\left|n_{1}^{p}(1), \ldots, n_{1}^{p}(N), n_{2}^{p}(1), \ldots, n_{2}^{p}(N)\right\rangle$

Hay que notar que la degeneración interna disponible en cada uno de los dos estados atómicos es incluida en la definición de la base $\left|n_{1}^{p}(1), \ldots, n_{1}^{p}(A), n_{2}^{p}(1), \ldots, n_{2}^{p}(A)\right\rangle$. La base para los estados que representan a los fotones $\left(n_{a}, n_{b}\right)$ se escribe en función de

$$
\left|n_{a}, n_{b}\right\rangle=\frac{a^{\dagger n_{a}} b^{\dagger n_{b}}}{\sqrt{n_{a} ! n_{b} !}}|0\rangle
$$

y la función de onda de fotones y átomos como

$$
\left|n_{a}, n_{b}, n_{1}, n_{2}\right\rangle=\left|n_{a}, n_{b}\right\rangle \otimes\left|n_{1}, n_{2}\right\rangle
$$

Se diagonalizó el Hamiltoniano de la Ecuación (3.1) en la base (3.9) bajo las condiciones

$$
\begin{array}{rlrl}
n_{0}+n_{1}+n_{2} & =A, & \\
n_{a}-n_{b}+n_{1}-A & =L & & (\text { configuración } \Xi), \\
n_{a}+n_{b}+n_{1}-A & =L & & (\text { configuración } \Lambda), \\
n_{a}+n_{b}-n_{1}-N & =L & & (\text { configuración } V) .
\end{array}
$$

Donde $A$ es el número de átomos.

\subsection{Resultados y discusión}

En lo que sigue se presentarán los resultados que se obtuvieron en el estudio del sistema descripto en la Sección 3.2. Se diagonalizó el Hamiltoniano de la Ecuación (3.1) y se calculó la evolución temporal de los operadores relevantes del sistema en la misma forma que se detalla en la Sección 5.2. Se tomó el espaciamiento de niveles atómicos que corresponden al ${ }^{87} R b$ (Brattke et al., 1998), que se detalla en la Figura 3.2. La energía del sector fotónico del Hamiltoniano se fijó en el caso de 

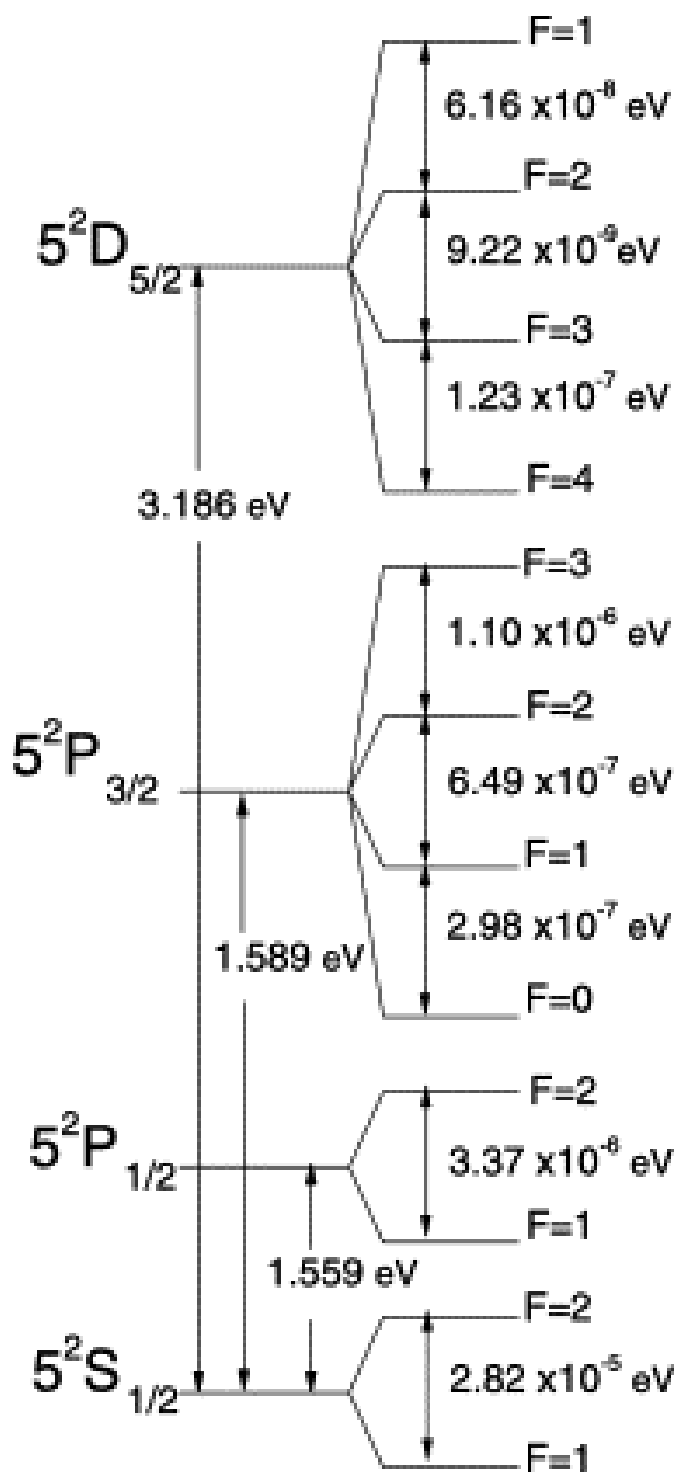

Figura 3.2: Esquema de niveles para los átomos de ${ }^{87} R b$. 
resonancia (Brattke et al., 1998). Para el estado inicial del sector fotónico se supuso un producto de estados coherentes

$$
\left|z_{a} z_{b}\right\rangle=N e^{z_{a} a^{\dagger}} e^{z_{b} b^{\dagger}}|0\rangle
$$

con $\left|z_{a}\right|^{2}=\left\langle n_{a}\right\rangle$ y $\left|z_{b}\right|^{2}=\left\langle n_{b}\right\rangle$. Se consideró, para el sector átomo-fotón del Hamiltoniano, los valores $g_{1}=0,025$ y $g_{2}=0,1 \mathrm{eV}$ para los esquemas $\Lambda$ y $V$, y $g_{1}=0,025$ y $g_{2}=0,3 \mathrm{eV}$ para el caso $\Xi$ (Reboiro et al., 2007). Los cálculos se realizaron para sistemas con $A=2,3$ y 5 átomos.

En la Figuras (3.3-3.5), se muestran los resultados para la evolución temporal del parámetro de squeezing $Q\left(S_{z}, S_{+}\right)$(que se detalló en la Sección 2.2.3), para diferentes tipos de interacción átomo-fotón con (y sin) el término de dipolo-dipolo de interacción (Ecuación (3.4)).

La Figura 3.3 muestra los resultados obtenidos para el esquema de interacción $\Lambda$ (Zhu and Lin, 1996; Wang, 2009; Cardimona et al., 2009; Barberis-Blostein and Bienert, 2009). Esta configuración se realiza tomando los niveles ${ }^{5} S_{1 / 2}(F=2)$, ${ }^{5} S_{1 / 2}(F=1)$ y ${ }^{5} P_{3 / 2}(F=2)$, de la Figura 3.2 , como los estados $|0\rangle,|2\rangle$ y $|1\rangle$ de la Figura 3.1(a). Los gráficos (a) y (b) corresponden a un sistema con $A=2$ átomos, los gráficos (c) y (d) corresponden a un sistema con $A=3$ átomos y los gráficos (e) y (f) a $A=5$ átomos respectivamente. Los estados iniciales fotónicos corresponden $\mathrm{a}\left\langle n_{a}\right\rangle=\left\langle n_{b}\right\rangle=2$, para los gráficos (a), (c) y (e), y $\left\langle n_{a}\right\rangle=\left\langle n_{b}\right\rangle=6$ para los gráficos (b), (d) y (f). Las curvas que se muestran en la Figura 3.3 están etiquetadas por la constante de interacción $g(g=0,0 e V$, y $g=0,05 e V)$. La inclusión de la interacción dipolo-dipolo conduce a valores grandes de $Q\left(S_{z}, S_{+}\right)$, lo cual significa que se reduce la posibilidad de generar compresión en incerteza en el sistema atómico. Los efectos son relativamente grandes para valores pequeños del número medio de fotones.

La Figura 3.4 exhibe los resultados cuando la configuración $\Xi$ (ver Figura 3.1(b)) es considerada (Van Kampen et al., 2000). El esquema efectivo incluye el estado ${ }^{5} S_{1 / 2}$, como el estado de menor energía $(|0\rangle)$, el estado ${ }^{5} P_{3 / 2}$ como el estado intermedio $(|1\rangle)$, y el estado ${ }^{5} D_{5 / 2}$ como el estado superior $(|2\rangle)$. Se supuso que los átomos se encuentran inicialmente en el estado fundamental. Para que a partir de 
este estado inicial se obtenga compresión en incerteza, se incrementó la asimetría en la interacción átomo-fotón $\left(g_{1}=0,025 \mathrm{eV}, g_{2}=0,3 \mathrm{eV}\right)$, en comparación con la configuración $\Lambda$ que fue de $\left(g_{1}=0,025 \mathrm{eV}, g_{2}=0,1 \mathrm{eV}\right)$. Se puede observar que la inclusión del término de interacción dipolo-dipolo, para este esquema, tiene un efecto menor, mientras que el incremento en media del número de fotones, en el estado inicial, tiende a lavar el del sector dipolar de la interacción y mejora ligeramente la compresión en incerteza atómica. En la Figura 3.5 se investigó la respuesta en el esquema $V$ (Agarwal and Patnaik, 2001; Kuzmich et al., 1997; Tan et al., 2009) (ver Figura 3.1(c)).Se tomó el estado ${ }^{5} S_{1 / 2}$, como el estado de menor energía y los estados ${ }^{5} P_{3 / 2}$ y ${ }^{5} P_{5 / 2}$ como los superiores (Agarwal and Patnaik, 2001). Se tomó, inicialmente, a todos los átomos en el estado ${ }^{5} P_{5 / 2}$, ya que, si se los tomaba en el estado ${ }^{5} S_{1 / 2}$ no se obtenía squeezing. En los gráficos (a) a (f)se muestran los resultados obtenidos con los mismo parámetros que en las Figuras 3.3 y 3.4. En este caso la aparición de squeezing se restringe solo a un breve intervalo de tiempo. La inclusión de la interacción dipolar produce el mismo efecto que en las configuraciones previas, idem con el incremento del número medio de fotones en el estado inicial.

Los resultados anteriores sugieren que, para las configuraciones estudiadas en átomos de tres niveles, la aparición de squeezing depende de varias condiciones físicas. En primera instancia, se puede decir que para las configuraciones $\Lambda$ y $\Xi$ la ocurrencia de squeezing es evidente. Por otro lado, para la configuración $V$ el squeezing atómico no aparece tan claramente si exploramos los valores de las constantes de acoplamiento, número de átomos y fotones. Las características más evidentes provienen de las configuraciones $\Lambda$ y $\Xi$ aparentemente indicando que, en ausencia de interacciones dipolo-dipolo, las variaciones en el número de átomos no afectan los observables de espín. En la misma condición $(g=0)$ el incremento en el número de fotones tiende a favorecer la aparición del fenómeno de squeezing en la configuración $\Xi$, y apenas modifica la evolución temporal de los observables de espín en la configuración $\Lambda$. Respecto al efecto de la interacción dipolar, se puede decir que esta no afecta al squeezing atómico en la configuración $\Xi$, pero se vuelve crucial destruyendo los patrones en la configuración $\Lambda$. 
Para dar una mirada física a esta situación volveremos a analizar los aspectos de simetría envueltos en los cálculos. Los números conservados en las bases son combinaciones lineales de la ocupación de niveles atómicos, número de fotones y número de átomos, como se explicó en la Sección 3.2.1. Si nos restringimos a dos casos donde se obserba el feómeno de squeezing atómico, esto es para las configuraciones $\Xi$ y $\Lambda$, desde el análisis numérico, se vuelve evidente que para la configuración $\Xi$ la relación $n_{0}>n_{1} \geq n_{2}$ cambia a $n_{0}>n_{2} \geq n_{1}$ cuando el número de fotones se incrementa. Para el esquema $\Lambda$ la relación $n_{0}>n_{1}>n_{2}$ se mantiene cuando el número de fotones se incrementa.

Uno puede trasladar estas relaciones entre ocupaciones de niveles en términos de la dependencia temporal del valor esperado de la componente $S_{z}$ del espín y sus fluctuaciones. Para el esquema $\Xi$ el incremento del número de fotones produce un incremento del valor absoluto de la componente $z$ del espín, independientemente de los efectos dipolares, y las fluctuaciones de espín decrecen. Para el esquema $\Lambda$, bajo las mismas condiciones, el valor absoluto de la componente $z$ del espín decrece y sus fluctuaciones aumentan ligeramente.

En cuanto al valor absoluto del operador $S_{+}$, este se incrementa (esquema $\Xi$ ) o permanece relativamente constante (esquema $\Lambda$ )con y sin interacciones dipolares, al incrementar el número de fotones. Todo esto se refleja sobre la dependencia temporal del parámetro de squeezing mostrados en la Figura 3.3 y 3.4. En cada caso, la tendencia se ve favorecida por la asimetría de la interacción entre el espín de los componentes de los niveles atómicos. La modificación de las condiciones iniciales pueden entonces afectar estos comportamientos en la forma que se ha explicado al comienzo de la sección.

\subsection{Conclusiones del capítulo}

En esta sección se estudió la compresión de la incerteza atómico en un sistema de átomos de tres niveles interactuando con dos modos del campo electromagnético. Se consideró los distintos esquemas de configuración $(\Lambda, \Xi$ y $V)$. Se realizaron cálculos 
con (y sin) la inclusión de interacciones dipolo-dipolo entre átomos. De los resultados se puede concluir que:

a) Para la interacción átomo-fotón, sin incluir el término dipolo-dipolo, se obtiene compresión en la incerteza atómica para las configuraciones $\Lambda$ y $\Xi$.

b) La inclusión de las interacciones dipolo-dipolo actúa en contra de la aparición del fenómeno de squeezing atómico.

c) El incremento del valor medio de fotones, en el estado inicial, disminuye el efecto de las interacciones dipolo-dipolo. 


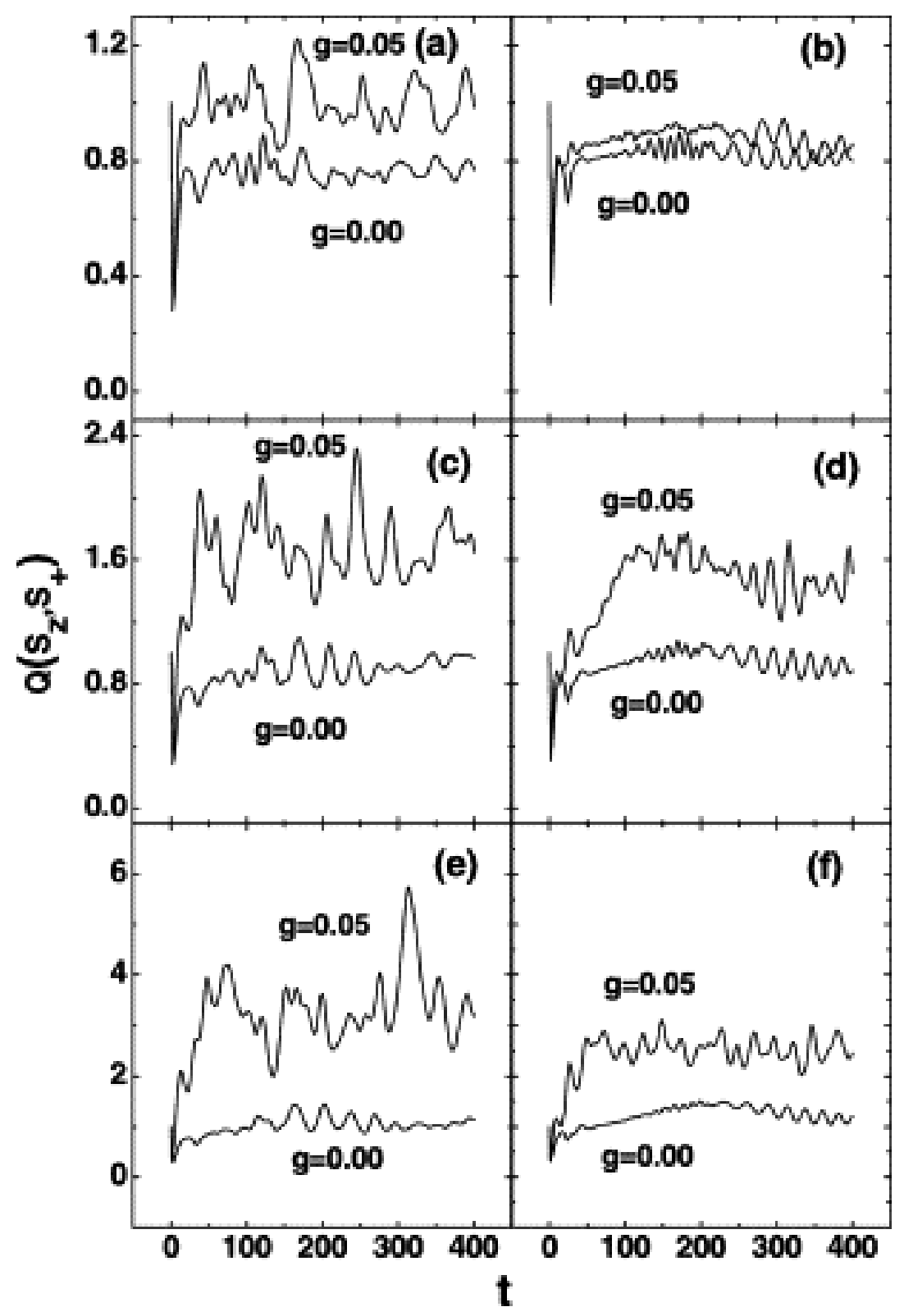

Figura 3.3: Parámetro de Squeezing $Q\left(S_{z}, S_{+}\right)$en función del tiempo. Los fotones y átomos interactúan mediante un esquema $\Lambda$. Para las constantes de acoplamiento del sector fotón-átomo del Hamiltoniano, se tomaron los valores de $g_{1}=0,025 \mathrm{eV}$ y $g_{2}=0,1 \mathrm{eV}$. El valor de la constante de acoplamiento del sector dipolo-dipolo del Hamiltoniano $g[e v]$ se indica en la figura. Los gráficos (a) y (b) muestran los resultados para un sistema de dos átomos, los gráficos (c) y (d) muestran los resultados para un sistema de tres átomos, y (e),(f) los de un sistema de cinco átomos. En todos los casos los átomos se encuentran en el estado fundamental. El valor medio de los fotones se fijó en $\left\langle n_{a}\right\rangle=\left\langle n_{b}\right\rangle=2$ (casos (a),(c) y (e)) y en $\left\langle n_{a}\right\rangle=\left\langle n_{b}\right\rangle=6$ (casos (b),(d) y (f)). 


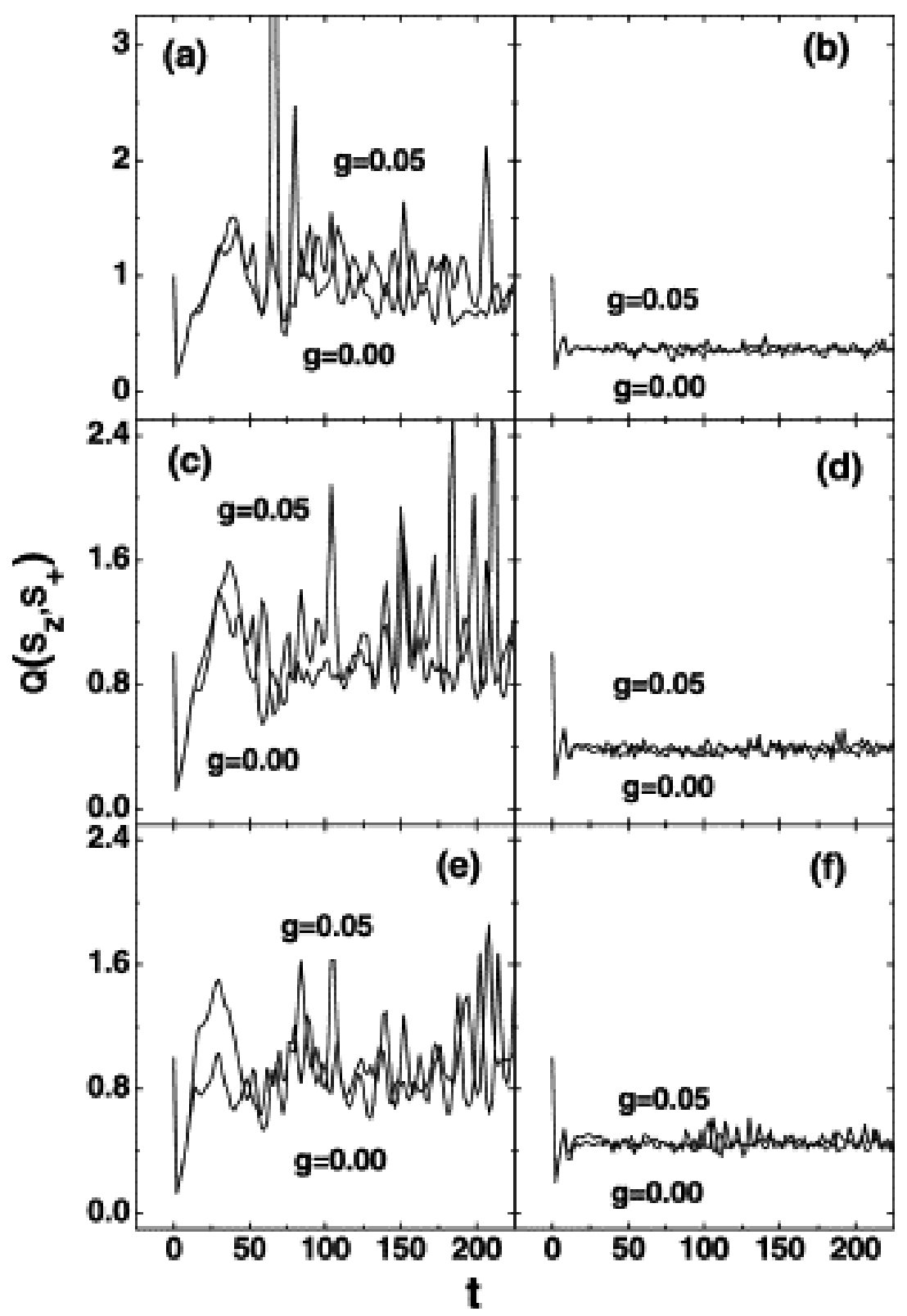

Figura 3.4: Parámetro de Squeezing $Q\left(S_{z}, S_{+}\right)$como función del tiempo para la configuración $\Xi$. Los valores de las contantes de acoplamiento del sector átomo -fotón del Hamiltoniano se fijaron en: $g_{1}=0,025 \mathrm{eV}$ y $g_{2}=0,1 \mathrm{eV}$. Los resultados se presentan en el mismo orden que en la Figura 3.3. 


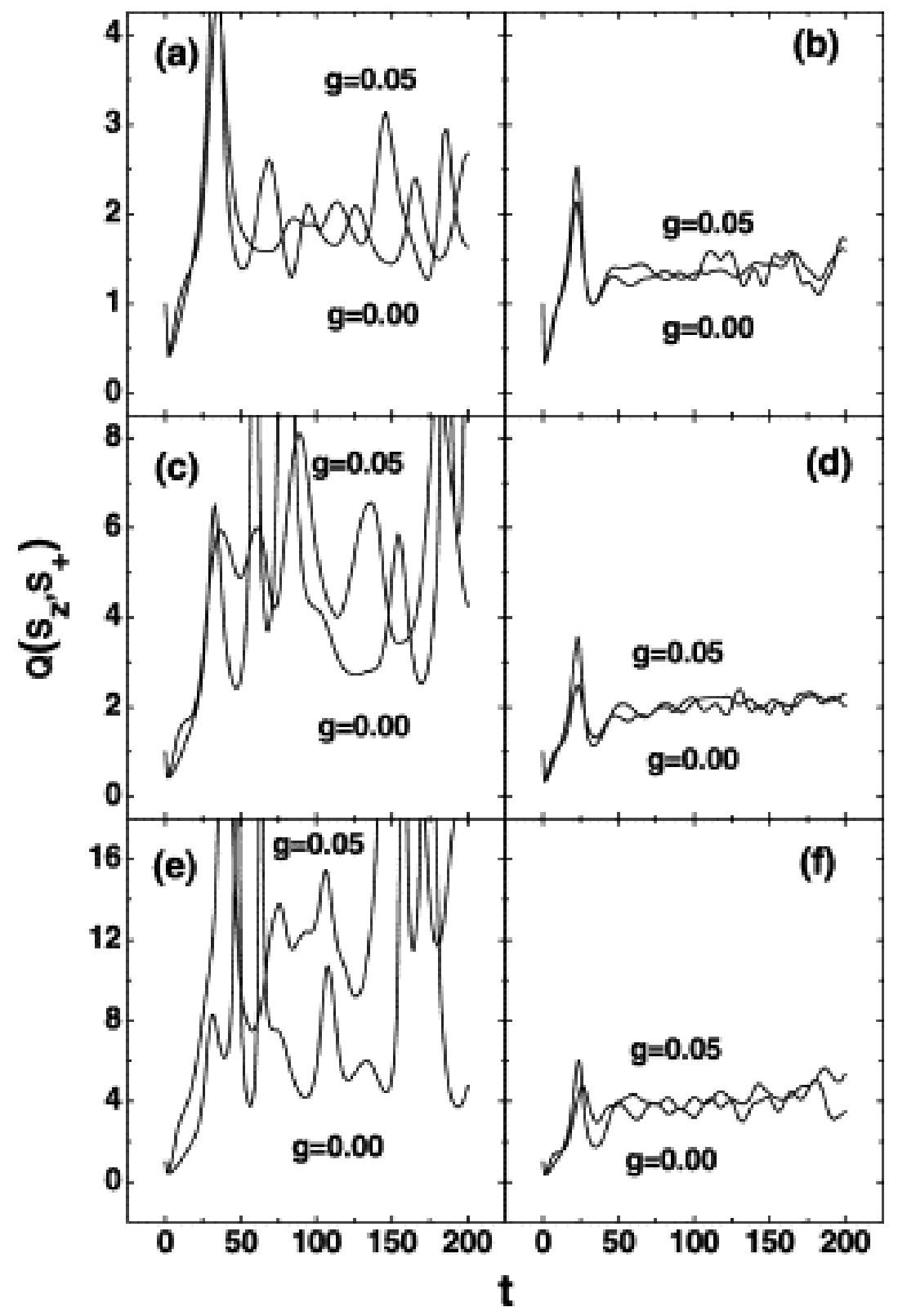

Figura 3.5: Parámetro de Squeezing $Q\left(S_{z}, S_{+}\right)$como función del tiempo para la configuración $V$. Los valores de las contantes de acoplamiento fueron fijadas en: $g_{1}=0,025 \mathrm{eV}$ y $g_{2}=0,1 \mathrm{eV}$. Los resultados fueron presentados de la misma forma que en la Figura 3.3. 


\section{Capítulo 4}

\section{Espín squeezing en presencia de disipación}

En este Capítulo se estudia la transferencia de espín entre fotones y niveles atómicos localizados para una cadena de átomos de dos niveles. El Hamiltoniano del sistema es modelado por un término de radiación y por interacciones átomo-átomo y fotón-átomo. Se han tenido en cuenta los efectos de disipación correspondientes al ancho de línea de los fotones mediante la inclusión de interacciones de intercambio fotón-átomo con constante de interacción compleja. Se observa que la compresión en la incerteza de espín es suprimida por la disipación. Los resultados que se presentan en este Capítulo corresponden a arreglos de átomos de Rubidio excitados por un láser de GaAlAs.

\subsection{Introducción}

El estudio de sistemas de espines, como laboratorios de efectos mecánico cuánticos, fue impulsado al considerar un sistema de $s=1 / 2$ como q-bits, siendo estos las unidades primordiales de la computación cuántica (Kok et al., 2007; Imai, 2006). Otro campo de interés, en conexión con los sistemas de espines, es la óptica cuántica (Scully and Zubairy, 1997; Peng and Li, 1998; Klauder and Sudarshan, 2006), donde se han registrado notables avances en la transferencia de espín (Furusawa and Takei, 2007; Takei et al., 2005; Sherson et al., 2006; Yonezawa et al., 2007; Lyakhov et al., 2007; Brey et al., 2007; Rabl and Zoller, 2007; Campos Venuti et al., 
2007; Romero-Isart et al., 2007; Joo et al., 2006; Fermani et al., 2007). A todo este conjunto de resultados me referiré como el campo de investigación del espín squeezing (Walls and Zoller, 1981; Prakash and Kumar, 2005; Kitagawa and Ueda, 1993; Drummond and Ficek, 2004; Cerf et al., 2007). En general, la interacción entre luz localizada y los niveles atómicos, producen transferencia de espín entre ellos (Lyakhov et al., 2007; Campos Venuti et al., 2007), y desviaciones de los valores esperados, en comparación con los valores dados por la conmutación estándar y las relaciones de incertidumbre (Walls and Zoller, 1981; Prakash and Kumar, 2005; Kitagawa and Ueda, 1993; Drummond and Ficek, 2004). Como se vió en el Capítulo 1, existe evidencia de compresión de incertezas de espin en sistemas atómicos en presencia de interacciones espín-fotón. En la referencia (Reboiro et al., 2007) se analiza con detalle la competencia entre las condiciones iniciales, para el caso de una cadena de $s=1 / 2$. Entre los problemas abiertos en el campo de la transferencia de espín, se puede mencionar la persistencia de la orientación del espín, y el resurgimiento de la alineación del espín (Romero-Isart et al., 2007; Lyakhov et al., 2007; Brey et al., 2007; Rabl and Zoller, 2007; Drummond and Ficek, 2004). En el reciente trabajo de Hagehstein y Chaudhary (2008) se investiga la atenuación de la transferencia de espín en presencia de disipación. La forma usual de estudiar el problema de la disipación en este tipo de sistemas, se puede encontrar en (Barnett and Knight, 1986). La descripción del fenómeno se basa en la utilización de la teoría de interacción entre radiación y espines. Hagelstein y Chaudhary (2008) consideran sistemas de dos niveles acoplados con un oscilador, y tienen en cuenta efectos disipativos debido al ancho de linea del modo de la cavidad mediante la inclusión de un término de interacción entre este y los átomos del sistema, con constante de acoplamiento compleja. Los resultados de (Hagelstein and Chaudhary, 2008) son muy interesantes, ya que los autores muestran que la inclusión de disipación incrementa la fracción de energía que se transfiere. Este efecto reportado es independiente del modelo utilizado para dar cuenta de la disipación, y esto abre la posibilidad de varias aplicaciones, la más destacada es la de fusión entre iones livianos (Hagelstein et al., 2006). En este capítulo se tomó el trabajo de (Hagelstein and Chaudhary, 2008) como motivación 
para estudiar los cambios debidos a los términos disipativos, en la persistencia de la compresión de la incerteza de espín de un modelo con interacción espín-espín. Estas interacciones no fueron consideradas en (Hagelstein and Chaudhary, 2008) y pueden ser de importancia, en particular, en los mecanismos de transferencia de espín (Lyakhov et al., 2007; Brey et al., 2007; Rabl and Zoller, 2007; Campos Venuti et al., 2007; Romero-Isart et al., 2007).

En este capítulo se estudian las condiciones de generación de squeezing atómico y la persistencia del mismo en un arreglo de átomos de rubidio, sistema recientemente analizado en (Echanis and et al., 2005). Además de las características ya comentadas, la relación entre el squeezing de espín y entrelazamiento fue estudiada recientemente en (Deb et al., 2006). Deb et al.(2006) consideraron un sistema de $N$ sistemas de átomos de dos niveles interactuando con un campo de radiación en una cavidad dispersiva. Entre los resultados mencionados en (Deb et al., 2006) se encuentra una reducción casi completa del ruido para un sistema de dos átomos en una cavidad con gran $Q$. En acuerdo con lo visto en el Capítulo 2 y en (Reboiro et al., 2007), los autores de (Deb et al., 2006) encontraron que el grado de squeezing se deteriora si el número de átomos en el estado excitado se incrementa. El objetivo de este Capítulo fue detallar la búsqueda de este tipo de efectos en sistemas como los estudiados en (Sørensen and Mølmer, 1999).

Este Capítulo se organizó de la siguiente forma. En la Sección 4.2 se presenta el Hamiltoniano que describe la interacción entre átomos y el campo de radiación. Luego presentan los resultados obtenidos para el caso de un arreglo de átomos de rubidio bajo diferentes parámetros del modelo (Sección 4.3). Para concluir, se presentan las conclusiones del capítulo en la Sección 4.4.

\subsection{Formalismo}

En esta sección se introducirá el Hamiltoniano del sistema, la base empleada y se describirá el método utilizado para resolver el problema de autovalores. La dependen- 
cia con las condiciones iniciales se discuten en la Sección 4.3. Se consideraron como estados iniciales, el productos de estados atómicos y de campos de radiación. Para el estado inicial atómico, se utilizaron estados coherentes de espín (Sección 1.2) y para estado inicial fotónico también se consideró un estado coherente bosónico (Apéndice B.2). La Sección 4.2.3 se utiliza para fijar la definición del parámetro de squeezing atómico utilizada (Kitagawa and Ueda, 1993).

\subsubsection{Hamiltoniano}

El sistema esta compuesto por cadenas localizadas de átomos de dos niveles activos y campos de radiación. Los átomos de cada sitio de la cadena interactúan en forma independiente con el campo de radiación. Esta situación que se ha modelado corresponde, aproximadamente, a la que se encuentra en trampas ópticas de iones. La interacción átomo-átomo es la descripta por la interacción espín-espín entre niveles atómicos. El Hamiltoniano resultante es

$$
\begin{aligned}
H & =H_{0}+H_{c a d}+H_{\text {int }} \\
H_{0} & =\hbar \omega\left(a^{\dagger} a+\frac{1}{2}\right)+\sum_{i} \epsilon(i) S_{z}(i) \\
H_{c a d} & =\sum_{i \neq j} \gamma_{i j} S_{z}(i) S_{z}(j)+\sum_{i \neq j} \eta_{i j}\left(S_{+}(i) S_{-}(j)+S_{-}(i) S_{+}(j)\right), \\
H_{\text {int }} & =\frac{1}{2} \sum_{i} \lambda_{i}\left(S_{+}(i)+S_{-}(i)\right)\left(a^{\dagger}+a\right),
\end{aligned}
$$

$H_{0}$ incluye la parte no perturbativa del campo (libre) de radiación, de frecuencia $\omega$ y de la componente de espín de los átomos en la dirección $z$. La posición de los átomos en la cadena, es indicada por el índice $i$. $H_{\text {cad }}$ es el término que describe la interacción entre espines atómicos situados en diferentes sitios de la cadena. La interacción entre las componentes $z$ del espín y la interacción entre las componentes $x$ e $y$ tienen diferentes intensidades, $\gamma_{i j}$ y $\eta_{i j}$ respectivamente. $H_{i n t}$ es el término de interacciones entre los fotones y los niveles atómicos pesadas por la constante $\lambda_{i}$. A este Hamiltoniano se le agregó un término disipativo de la forma

$$
H_{\text {dis }}=\frac{i}{2} \sum_{i} \lambda_{d}(i)\left(S_{+}(i)+S(i)_{-}\left(a^{\dagger}+a\right),\right.
$$


como sugiere (Hagelstein and Chaudhary, 2008). La justificación de este término se encuentra en teoría de campos, donde el acoplamiento de una partícula con una resonancia estrecha produce, al más bajo orden en teoría de perturbaciones, un término con la dependencia presentada en la Ecuación (4.5). La electrodinámica cuántica de un átomo de dos niveles interactuando con un modo de una cavidad, fue desarrollado por (Barnett and Knight, 1986). Esta conduce a términos de acoplamiento que son proporcionales al ancho del modo de la cavidad. En (Barnett and Knight, 1986) se muestra que la matriz densidad, es amortiguada en el tiempo por factores exponenciales que son proporcionales al ancho del modo de la cavidad.

El Hamiltoniano de la Ecuación (4.4) puede diagonalizarse en la base

$$
|k, n\rangle=\left|k_{1}, k_{2}, \ldots, k_{i}, \ldots, k_{N}, n\right\rangle=\prod_{i=1}^{N} S_{+}^{k_{i}}(i) \frac{a^{\dagger^{n}}}{\sqrt{n !}}|0\rangle, k_{i}=0,1 .
$$

En la expresión anterior $k$ es un arreglo de espines perteneciente al conjunto $\{k\}=$ $\left\{k_{1}, k_{2}, \ldots, k_{i}, \ldots, k_{N}\right\}$.

Los operadores $S_{ \pm}(i)=S_{x}(i) \pm i S_{y}(i)$ son los operadores escalera del $i$-ésimo sitio, que junto con el operador $S_{z}(i)$ son los generadores del álgebra $S U(2)$.

Los autovectores se escriben como combinaciones lineales de la base Ecuaciones (4.6)

$$
|\alpha\rangle=\sum_{\{k\}, n} c_{\alpha}(k, n)|k, n\rangle
$$

El termino $H_{\text {diss }}$ se trató perturbativamente en la base de los autoestados (Ecuación (4.7)), al más bajo orden, corrigiendo la energías y renormalizando los autoestados $|\alpha\rangle$. Con los autoestados se calculó, en forma análoga a lo realizado en la Sección 5.2 del Capítulo 2, los valores medios de los observables de espín en función del tiempo para diferentes condiciones iniciales.

\subsubsection{Condiciones iniciales}

Para este sistema se consideró un estado inicial, que no es un autoestado de $H$ y que puede describirse como producto directo del estado del campo electromagnético por el estado estado atómico,

$$
|I\rangle=|I\rangle_{f} \otimes|I\rangle_{a t}
$$


Donde $|I\rangle_{f}$ es el estado inicial fotónico y $|I\rangle_{a t}$, el estado inicial atómico. Se tomó como condición inicial para el campo de radiación un estado coherente (ver Apéndice B.2)

$$
|I\rangle_{f}=e^{|z|^{2} / 2} e^{z a^{\dagger}}|0\rangle
$$

$\operatorname{con}|z|^{2}=\left\langle a^{\dagger} a\right\rangle$

Para el estado inicial atómico se adoptó un estado coherente de espín (Sección 1.2)

$$
\begin{aligned}
|I\rangle_{a t} & =e^{z_{a t} S_{+}}|0\rangle, \\
& =\left(1+\left|z_{a t}\right|^{2}\right)^{-\frac{N}{2}} \sum_{m=0}^{N} \frac{z_{a t}^{m}}{m !} S_{+}^{m}|0\rangle,
\end{aligned}
$$

con

$$
z_{a t}=e^{-i\left(\phi_{0}-\pi\right)} \tan \left(\frac{\theta_{0}}{2}\right)
$$

y $S_{+}$es el operador colectivo de subida

$$
S_{+}=\sum_{l} s_{+, l}
$$

\subsubsection{Squeezing atómico}

El parámetro de squeezing atómico que se utiliza en este Capítulo es el mismo que se introdujo en el Capitulo 2 (Ecuación (1.69)), este se escribe

$$
\zeta^{2}=\frac{2\left(\Delta S_{\perp}\right)^{2}}{|<\mathbf{S}>|} .
$$

El cual nos dice que el sistema se encuentra comprimido en incerteza en la variable $S_{\perp}$ si $\xi^{2}<1$

\subsection{Resultados y discusión}

Se estudió la evolución temporal de los observables de espín del sistema, bajo la acción del Hamiltoniano (Ecuación (4.4)), utilizando el formalismo introducido en la Sección 5.2. Se consideró una cadena abierta con $N=5$ sitios. Los valores para $\epsilon(i)$ fueron tomados de los niveles atómicos $S$ y $P$ del ${ }^{87} R b$ esto es, $\epsilon(i)=1,589 \mathrm{eV}$ si $i$ es par, y $\epsilon(i)=1,82 \mathrm{eV}$ si $i$ es impar. La energía del fotón se fijo en $\hbar \omega=1,582 \mathrm{eV}$ 
que se corresponde con un láser de GaAlAs. Las constantes de acoplamiento $\gamma_{i j}$ y $\eta_{i j}$, del sector espín-espín del Hamiltoniano, se fijaron considerando la distancia entre espines $\left(\gamma_{i j}=0,01 \mathrm{eV}\right.$ si $|i-j| \leq 2, \gamma_{i j}=0$ caso contrario, y $\left.\eta_{i j}=2 \gamma_{i j}\right)$. Las constantes $\lambda_{i}$ y $\lambda_{d}(i)$ se fijaron en los valores $\lambda_{i}=0,022 \mathrm{eV}$ y $\lambda_{d}(i)=0,002 \mathrm{eV}$. Las condiciones iniciales para los átomos fueron estados de espín coherentes (Ecuación (4.10)), con $\phi_{0}=\theta_{0}=\pi / 4$.

Estos valores se tomaron como aproximaciones, cercanas a las que se encuentran en una situación realista. Sin embargo, esta es consistente con la información experimental para la diferencia de energía entre los niveles $S$ y $P$ de un átomo de $\mathrm{Rb}, \mathrm{y}$ las propiedades de un láser de GaAlAs con una línea de 1,582eV. Los valores de $\lambda_{i}$ y $\lambda_{d}(i)$ se eligieron en forma consistente con el tratamiento, en teoría de campos, de las interacciones entre radiación y niveles atómicos. A fin de evaluar el orden de magnitud de los efectos asociados con los diferentes términos del Hamiltoniano de la Ecuación (4.4), se realizaron cálculos cambiando gradualmente cada uno de los términos.

A continuación se presentará y discutirá los resultados para los valores de la componente z del espín de cada uno de los sitios de la cadena, el valor medio de las componentes del espín total $\left(\left\langle S_{x}\right\rangle,\left\langle S_{y}\right\rangle,\left\langle S_{z}\right\rangle\right)$ y el parámetro de squeezing atómico. Como primer paso se verificó que, para un Hamiltoniano restringido a una interacción puramente espín-espín entre sitios vecinos, la componente z del espín total y la componente $z$ de cada sitio fuera una constante de movimiento. La inclusión de vecinos mas lejanos, como es el caso que se analiza en este capítulo, rompe esa simetría. Los resultados de estos cálculos se muestran en la Figura 4.1, en la cual se ilustra en (a) la dependencia con el tiempo de $\left\langle S_{z}\right\rangle$ para cada átomo, (b) el resurgimiento (en el tiempo) de los los valores esperados para las componentes $x$ e $y$ del espín.

La Figura 4.2 muestra el resultado que se obtiene usando, al igual que en la Figura 4.1, solo el sector atómico del Hamiltoniano (Ecuación (4.4)). En (a),(b) y (c) de la Figura 4.2 se muestra la dependencia temporal de los valores esperados del factor de squeezing $\xi^{2}$, y la dependencia con el tiempo de los ángulos de orientación del espín medio total. Como se ve en las curvas de la Figura 4.2, la compresión en 
inceteza de espín atómico persiste en el tiempo, mostrando un comportamiento de resurgimiento, alcanzando un mínimo del orden de $\xi^{2}=0,7$.

El efecto de incluir la interacción con el campo de los fotones se muestra en la Figura 4.3. El Hamiltoniano usado para realizar los cálculos es el de la Ecuación (4.4), exceptuando el término de disipación, que ha sido omitido en este conjunto de cálculos. Hemos considerado un estado coherente para el sector fotónico (Ecuación (4.9)), con $|z|^{2}=10$. El resto de los parámetros del Hamiltoniano se comentaron anteriormente. En este caso el squeezing $\xi^{2}$ no persiste con el tiempo, y alcanza un mínimo $\operatorname{con} \theta=\pi / 2$. Se investigan los efectos asociados con el número de de fotones $\left(n_{b}=|z|^{2}\right)$ en el estado inicial, realizando cálculo para los casos $n_{b}=2,4,6$ y $8, \mathrm{y}$ comparando los resultados con los obtenidos con $n_{b}=10$. Es evidente, de las curvas mostradas en la Figura 4.3, que la interacción átomo-fotón destruye el squeezing inducido por las interacciones espín-espín. Este comportamiento persiste si el número de fotones en el estado inicial se cambia, esto es, el número de fotones en el estado inicial no influencia demasiado la dependencia temporal del squeezing.

Los resultados para el Hamiltoniano completo de la Ecuación (4.4), se muestran en la Figura 4.4, para el caso $n_{b}=2$. Los efectos de la disipación son notorios, dado que el squeezing es borrado en todo el intervalo temporal considerado en los cálculos. Además se observa que los valores esperados de la orientación del espín oscila entre $\theta=\pi / 2$ y $\theta=\pi$. La transferencia de espín desde el campo de los fotones por sitio de la cadena difiere drásticamente comparado con los resultados de la Figura 4.2, debido a que para este caso los niveles de cada sitio atómico están igualmente poblados. La misma conclusión respecto al efecto en el número de fotones puede ser señalada y sostenida aquí, por los resultados que se muestran en la Figura 4.5, que corresponde al caso $n_{b}=10$. En las Figuras 4.4(a) y 4.4(a) puede apreciarse que el squeezing desaparece para valores grandes de $t$. Respecto de la dependencia con la elección del estado inicial atómico, se han repetido los cálculos presentados cambiando los estados de espín coherente por uno en el cual todos los átomos se encuentran en el estado fundamental. Los resultados de estos cálculos, mostraron que el uso del estados de espín coherente es importante, para la aparición de la compresión 
en incerteza de espín, como lo es un estado coherente para el sector fotónico. Hay que remarcar que estos resultados concuerdan con los de (Echanis and et al., 2005). Básicamente se obtuvieron resultados similares del comportamiento del squeezing cuando se incorpora disipación. En ambos trabajos el squeezing es borrado por la disipación, aunque en el trabajo de (Echanis and et al., 2005), la disipación se incorpora a través del dispersión de fotones y en nuestro caso del acoplamiento entre el espín atómico y el ancho de línea del campo fotónico.

\subsection{Conclusiones del capítulo}

En este capítulo se calculó la dependencia temporal de los observables de espín en presencia de interacciones átomo-átomo y átomo-radiación. También se introdujo disipación mediante interacciones átomo-fotón con constante de acoplamiento compleja.

Los resultados, en relación con los observables de espín, son los siguientes:

a) La inclusión de la interacción átomo-átomo produce la mejora del factor de squeezing y, finalmente, es responsable de los efectos de resurgimiento;

b) La presencia del término de disipación limita la transferencia de espín del campo de radiación a los niveles atómicos;

c) El estado inicial atómico, en nuestro caso, un estado de espín coherente, es tan importante como el estado inicial del campo de radiación (también un estado coherente) en la producción de la alineación de espín;

d) El principal efecto entre las interacciones átomos-fotón y átomo-átomo es que ellos agregan incoherencia a pesar del número de fotones involucrado;

e) La dependencia de la transferencia de espín sobre los acoplamientos atómicos es evidente y abre la posibilidad modelar los efectos de la transferencia de espín por medio de redes atómicas, y/o trampas ópticas. 


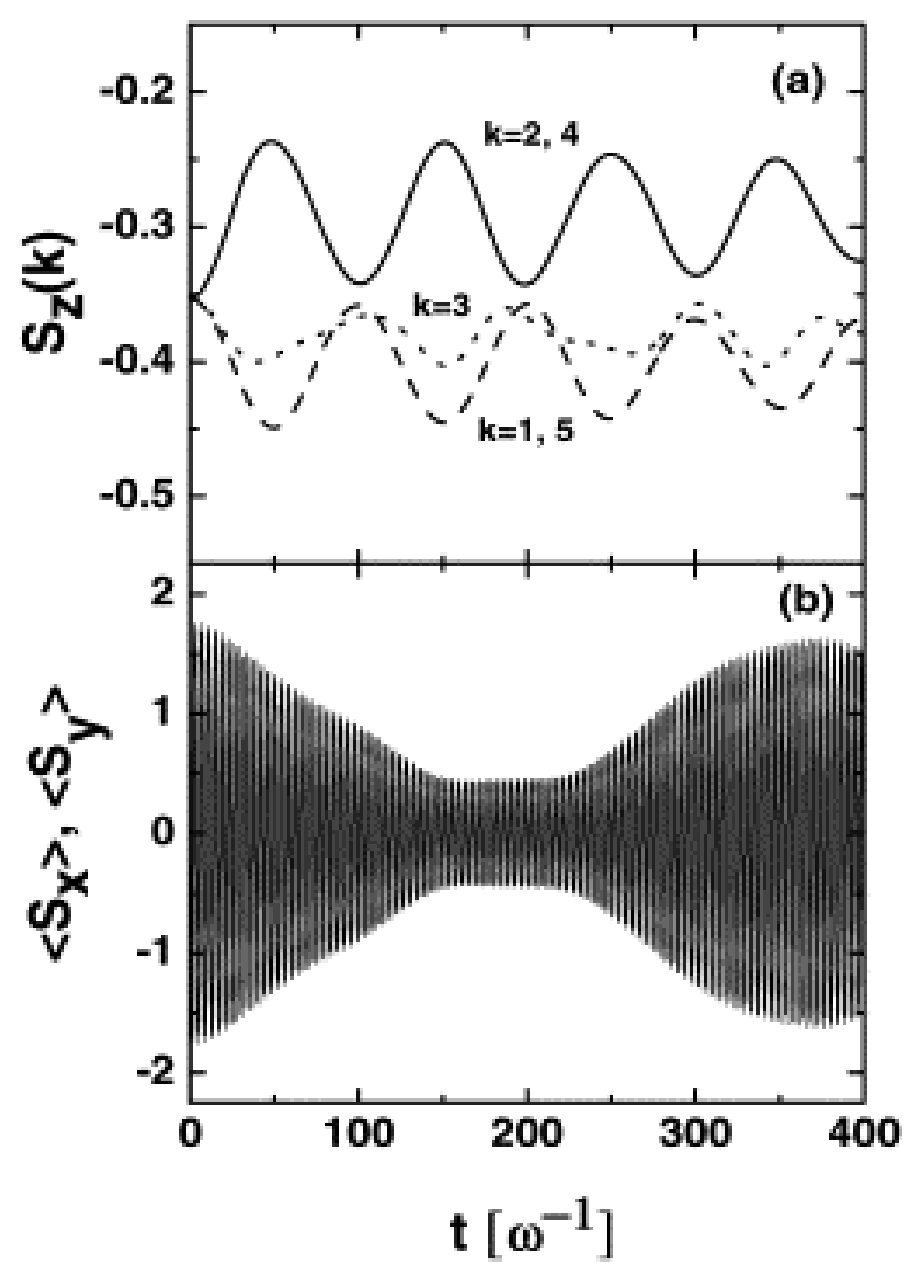

Figura 4.1: Valores esperados de las componentes de espín con un Hamiltoniano puramente atómico. Estos resultados corresponden a una cadena de 5 sitios en función del tiempo $t$ ( $t$ está dado en unidades arbitrarias). Los valores de la componente $z$ del espín total, $S_{z}$, están dados para cada uno de los sitios (a). La dependencia temporal de las componentes $S_{x}$ y $S_{y}$ del espín total se muestra en (b). El Hamiltoniano solo incluye términos atómicos (espín) del sistema y no incluye a la parte fotónica. El estado inicial utilizado es un estado de espín coherente (ver Ecuación (4.10)) y los parámetros del Hamiltoniano son los dados en el texto. 


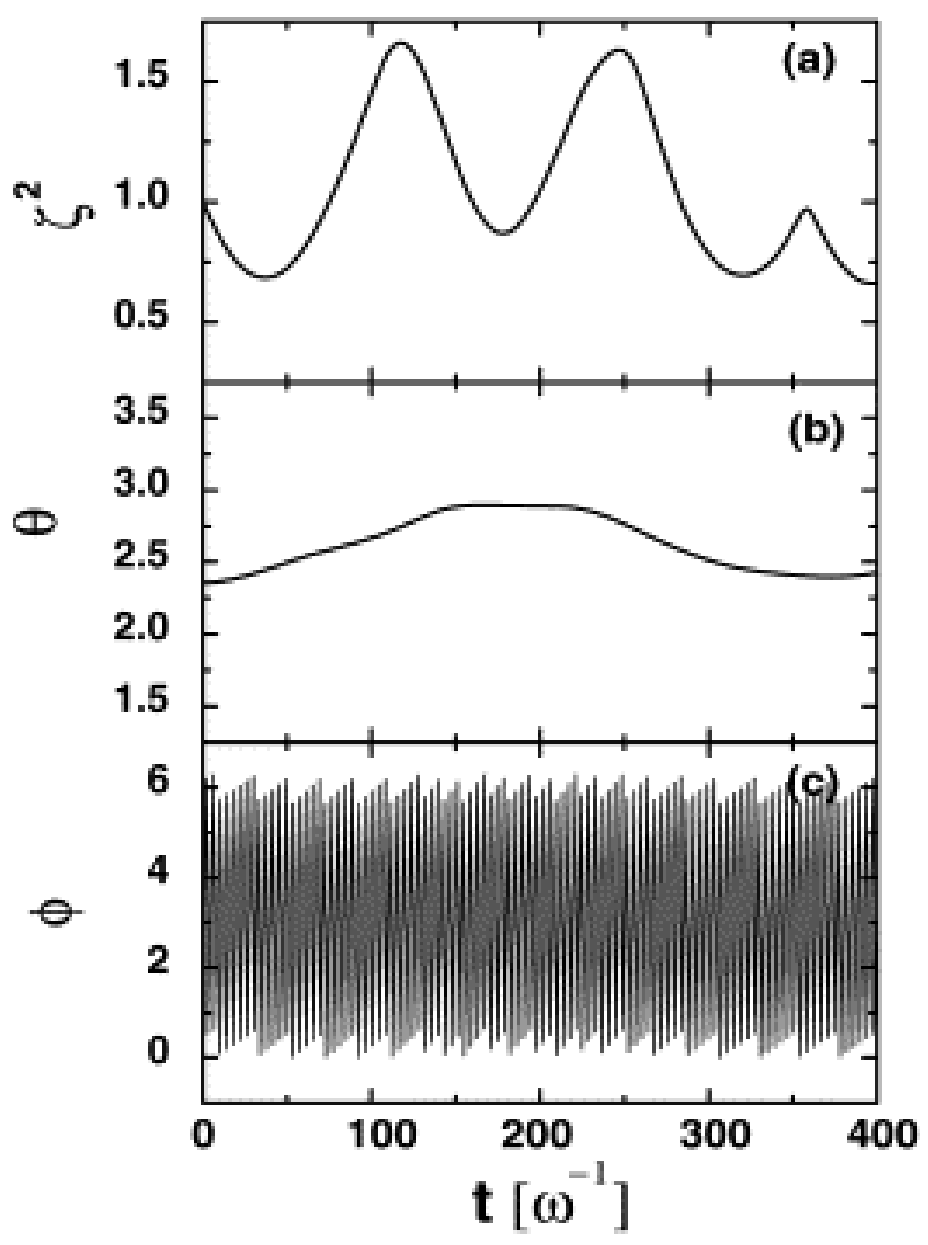

Figura 4.2: (a) Evolución temporal del factor de squeezing $\xi^{2}$, y en (b) y (c) los ángulos de orientación del vector espín medio $\langle S\rangle, \theta$ y $\phi$, respectivamente. Se consideró solo el término de interacción átomo-átomo. El estado inicial $|I\rangle_{a t}$ es un estado coherente Ecuación (4.10) con $\phi_{0}=\theta_{0}=\pi / 4$ 


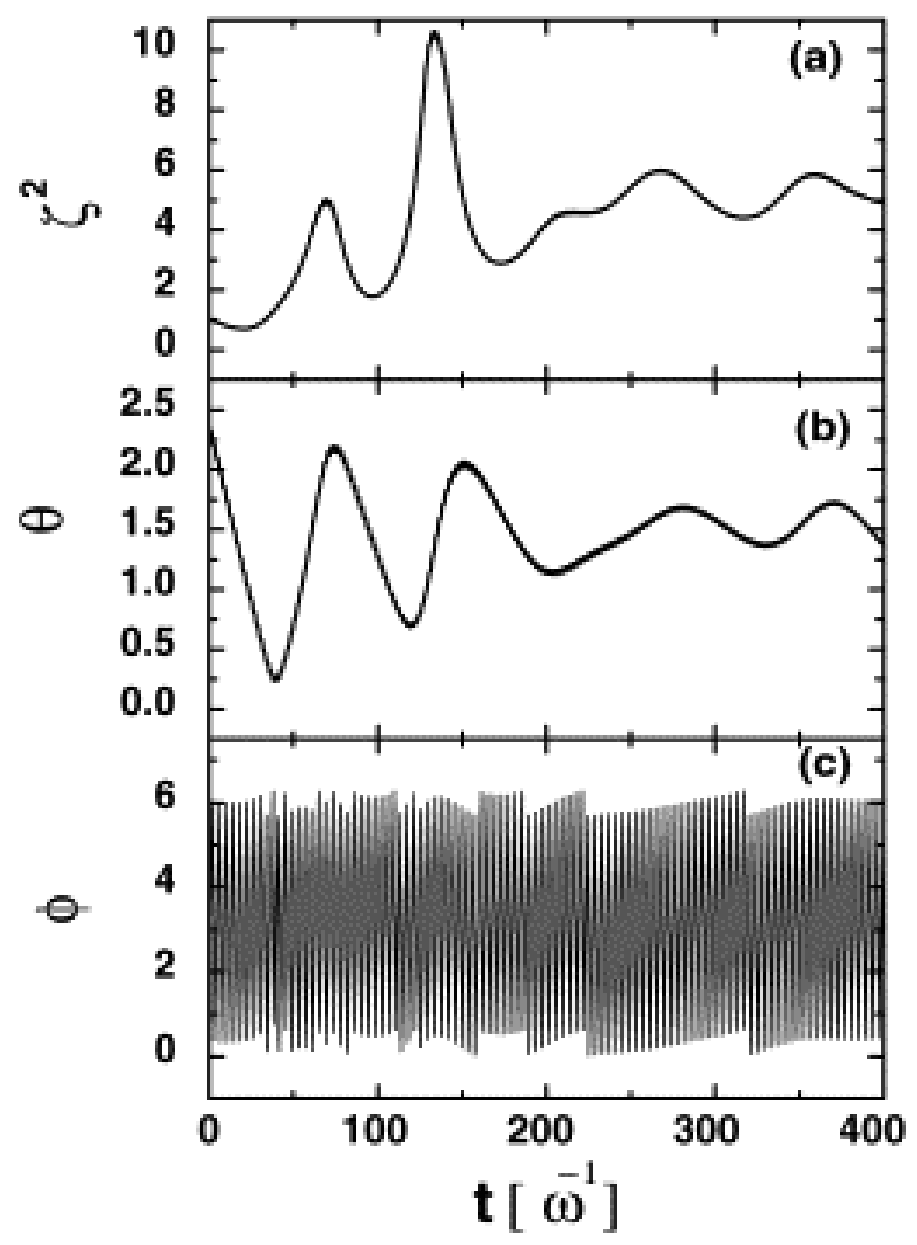

Figura 4.3: (a) Evolución temporal del factor de squeezing $\xi^{2}$, y en (b) y (c)los ángulos de orientación del vector espín medio $\langle S\rangle, \theta$ y $\phi$, respectivamente, en presencia de interacciones átomoátomo y átomo-fotón, sin disipación. El estado inicial para el sector fotónico es un estado coherente Ecuación (4.9) con 10 fotones $\left(n_{b}=\left|z^{2}\right|=10\right)$, y para la parte atómica $|I\rangle_{a t}$ se utilizó un estado coherente Ecuación (4.10) con $\phi_{0}=\theta_{0}=\pi / 4$. Los parámetros del Hamiltoniano son los dados en el texto. 


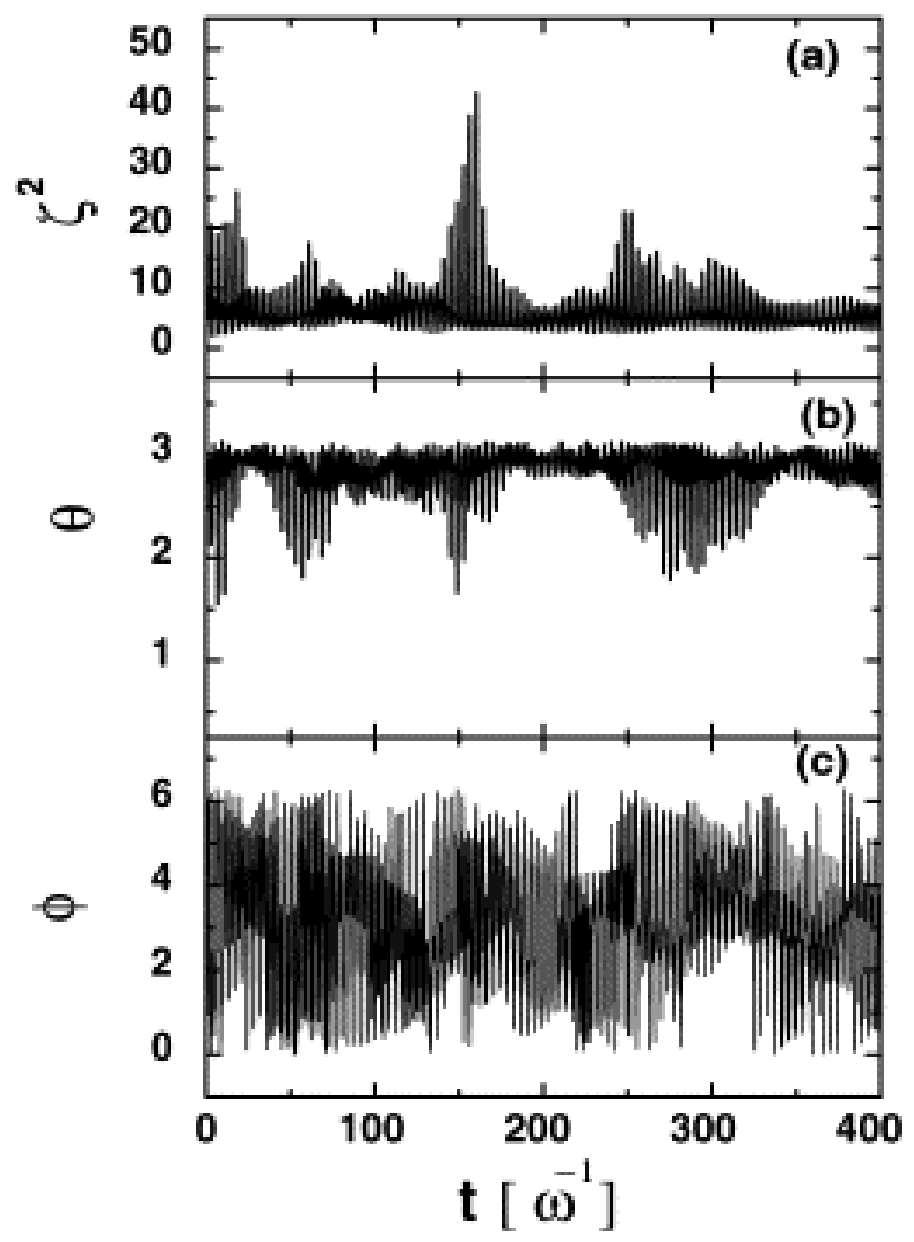

Figura 4.4: (a) Evolución temporal del factor de squeezing $\xi^{2}$, y en (b) y (c)los ángulos de orientación del vector espín medio $\langle S\rangle, \theta$ y $\phi$, respectivamente, en presencia de interacciones átomo-átomo y átomo-fotón, con disipación. El estado inicial para el sector fotónico y atómico es coherente con $\left(n_{b}=\left|z^{2}\right|=2\right)$ y $\phi_{0}=\theta_{0}=\pi / 4$. Los parámetros del Hamiltoniano son los dados en el texto. 


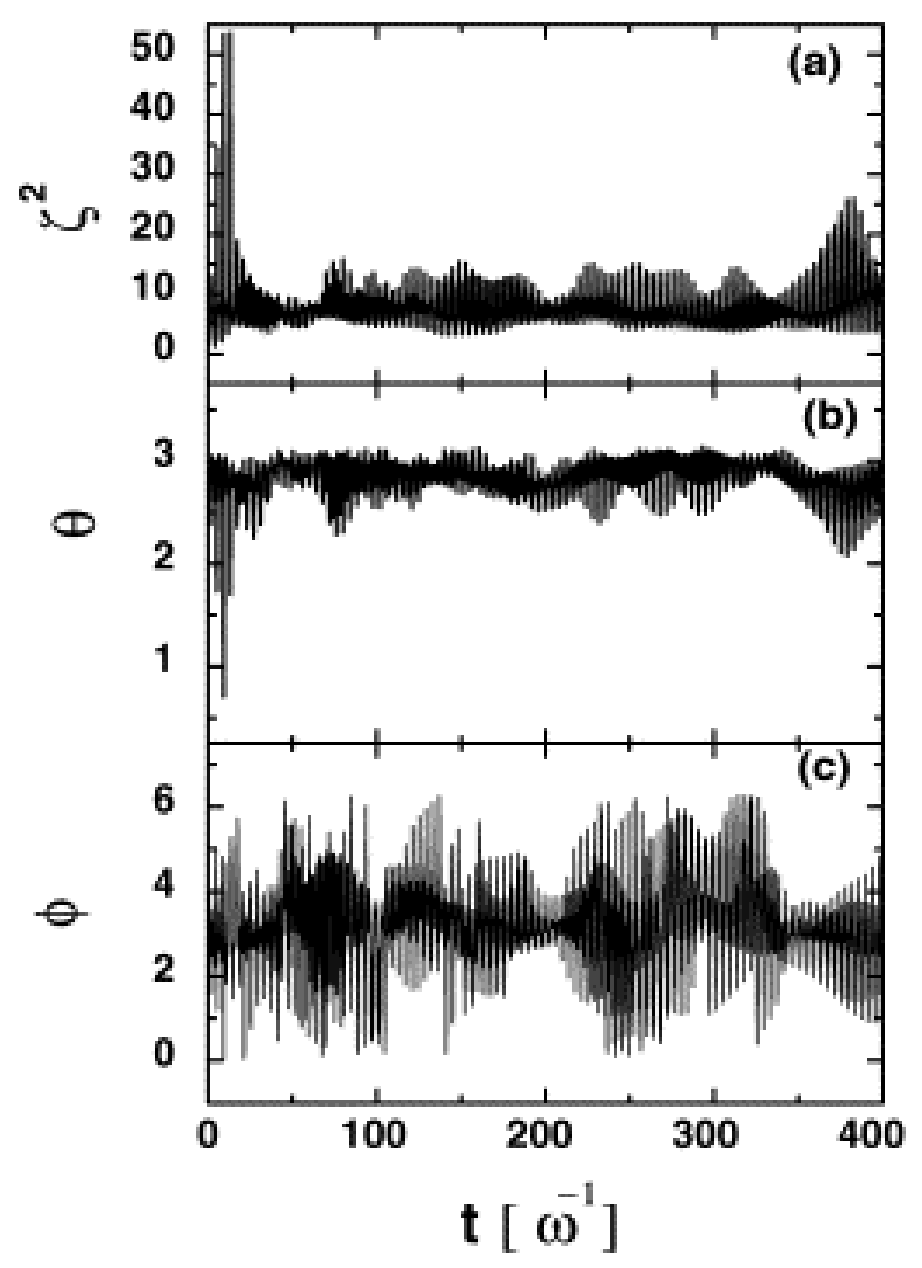

Figura 4.5: (a) Evolución temporal del factor de squeezing $\xi^{2}$, y en (b) y (c)los ángulos de orientación del vector espín medio $\langle S\rangle, \theta$ y $\phi$, respectivamente, en presencia de interacciones átomo-átomo y átomo-fotón, con disipación. El estado inicial para el sector fotónico y atómico es coherente con $\left(n_{b}=\left|z^{2}\right|=10\right)$ y $\phi_{0}=\theta_{0}=\pi / 4$. Los parámetros del Hamiltoniano son los dados en el texto. 


\section{Capítulo 5}

\section{Squeezing en una cadena de espines con interacciones de largo alcance dependiente del sitio}

En este capítulo se presentarán resultados numéricos y analíticos para el factor de squeezing $\xi^{2}$, en cadenas de $N$ espines atómicos $(s=1 / 2)$ abiertas modeladas con interacciones dependientes del sitio. Se estudió la evolución temporal del parámetro de squeezing, así como también la dependencia de este respecto del número de átomos y el tipo de interacción. Se encontró que las interacciones de largo alcance pueden optimizar el grado del espín squeezing.

\subsection{Introducción}

Como se mencionó en los Capítulos anteriores recientes desarrollos en computación y óptica cuántica han renovado el interés en estudiar sistemas de espines y sus interacciones (Kok et al., 2007; Imai, 2006; Scully and Zubairy, 1997; Peng and Li, 1998; Klauder and Sudarshan, 2006). En particular, el problema de reconstrucción de espín en mediciones cuánticas de no-demolición (Shaffman et al., 2009; Teper et al., 2008; Oblak, 2005) está estrechamente relacionado con los observables de espín squeezing (Hagelstein and Chaudhary, 2008; Nielsen and Mølmer, 2008; Echanis and et al., 2005; Korbicz et al., 2006). Sin embargo hay algunas cuestiones acerca del modelado y las propiedades físicas de los sistemas de espín que deben ser aclaradas para 
confirmar la afirmación de que las cadenas de espines son dispositivos adecuados para la transmisión de información cuántica. Entre ellas se seleccionó para este Capítulo la pregunta acerca de la persistencia de la orientación del espín en presencia de interacciones tipo espín-espín (Troinai, 2005; Affronte et al., 2004; Carretta et al., 2003; Sørensen and Mølmer, 1999; Wang, 2001). Se investigó la respuesta del factor de squeezing atómico de una cadena de espines con diferentes interacciones (Shastry, 1988; Haldane, 1988; Inozemtsev and Kuzemsky, 1991; Inozemtsev, 1995; Inozemtsev and Inozemtseva, 1991; Dittrich and Inozemtsev, 1997; Inozemtseva and Inozemtsev, 1997). Basado en los Capítulos previos se considerará una cadena anisotrópica, debido a que las anisotropías en las interacciones de las cadenas de espines, juegan un papel en la construcción del equilibrio entre los componentes del espín total. Para realizar este estudio se seleccionó de la literatura interacciones espín-espín periódicas, y Gausianas periódicas de largo alcance (Shastry, 1988; Haldane, 1988; Inozemtsev and Kuzemsky, 1991; Inozemtsev, 1995).

En el presente trabajo se calcularon observables de espín en función del tiempo y se estudio la compresión en incerteza de espín para un sistema de átomos de dos niveles distribuidos en los sitios de una cadena abierta. Los cálculos de los valores esperados para el espín se obtienen utilizando el formalismo de la matriz densidad desarrollado en la Sección 5.2.

La organización de este capítulo es la siguiente: El formalismo utilizado se describe en la Sección 5.2, los resultados y discusión se presentan en la Sección 5.3, y para finalizar se exponen las conclusiones en la Sección 5.4.

\subsection{Formalismo}

El marco de referencia, para el formalismo que se detallará a continuación, es el tratamiento de cadenas de espín $s=1 / 2$ antiferromagnéticas (ferromagnéticas) con interacciones dependientes del sitio (Shastry, 1988; Haldane, 1988). Los aspectos formales para su solución son bien conocidos (Inozemtsev and Kuzemsky, 1991; 
Inozemtsev, 1995), y se omitirán por razones de espacio. Esencialmente, uno debe definir el Hamiltoniano de la cadena, la base, y, a través de una diagonalización, se obtiene la solución exacta para un cierto número de átomos (Civitarese et al., 2009). Las siguientes subsecciones se centraran básicamente en los detalles específicos de la resolución para distintas interacciones. Respecto a la evolución temporal de observables, se utilizó el formalismo de la matriz densidad que se desarrollo en la Sección . Para el factor de squeezing se utilizó la definición de la Sección 1.3.2.

\subsubsection{Cadenas de espines asimétricas}

En lo que sigue se discutirán algunos ejemplos de sistemas de espín que son exactamente solubles por diagonalización. El espectro de cada átomo consiste en dos niveles de espín $s=1 / 2$. Por simplicidad se hablará del sistema como una cadena de espines $s=1 / 2$. Sin embargo, debido a que estamos hablando de sistemas de dos niveles, el término cadena de pseudo-espín sería más apropiado. La interacciones entre los átomos son del tipo espín-espín y se descompusieron en componentes, de una forma general, adoptando constantes de acoplamiento diferentes. Se escribió el el Hamiltoniano del sistema como

$$
H=\sum_{i \neq j=1}^{N} g(i, j)\left(\lambda_{x} s_{x, i} s_{x, j}+\lambda_{y} s_{y, i} s_{y, j}+\lambda_{z} s_{z, i} s_{z, j}\right) .
$$

La interacción de la Ecuación (5.1) es una generalización de la interacción entre espines de los sitios $\bar{S}_{i} \cdot \bar{S}_{j}$ pesada por acoplamientos direccionales.

$$
g_{\gamma}(i, j)=\lambda_{\gamma} g(i, j), \quad \gamma=x, y, z \quad i=1,2, \ldots, N
$$

Los operadores escalera de espín, $s_{ \pm, i}=s_{x, i} \pm i s_{y, i}$, y el operador $s_{z, i}$ obedecen las relaciones de conmutación de $S U(2)$, y el Hamiltoniano de la Ecuación (5.1) puede escribirse como

$$
H=\sum_{i \neq j=1}^{N} g(i, j)\left(\lambda_{-}\left(s_{+, i} s_{+, j}+s_{-, i} s_{-, j}\right)+\lambda_{+}\left(s_{+, i} s_{-, j}+s_{-, i} s_{+, j}\right)+\lambda_{z} s_{z, i} s_{z, j}\right)
$$


con $\lambda_{ \pm}=\frac{1}{2}\left(\lambda_{x}+\lambda_{y}\right)$. El producto de estados

$$
|m\rangle=\left|k_{1}, k_{2}, \ldots, k_{i}, \ldots, k_{N}\right\rangle=\prod_{i=1}^{N} s_{+, i}^{k_{i}}|0\rangle, \quad k_{i}=0,1,
$$

define una base donde el Hamiltoniano (Ecuación (5.3)) puede ser diagonalizado; el índice $k_{i}$ etiqueta el estado del $i$-ésimo átomo y los valores $k_{i}=1,0$ están asociados con el estado fundamental $s_{z}=-1 / 2$ y el primer excitado $s_{z}=1 / 2$, de cada átomo respectivamente. El estado $|0\rangle$ es el vacío, y por definición del mismo en el estado $|0\rangle$ todos los átomos se encuentran en el estado fundamental o de menor energía $\left(s_{z}=-1 / 2\right)$. La matriz de los elementos del Hamiltoniano, de la Ecuación (5.3), en la base (Ecuacion(5.4)) son

$$
\begin{aligned}
\left\langle m^{\prime}|H| m\right\rangle=\quad & \sum_{i \neq j=1}^{N} g(i, j)\left(\lambda_{-} \delta\left(k_{i}+1, k_{i}^{\prime}\right) \delta\left(k_{j}+1, k_{j}^{\prime}\right)\right. \\
& +\lambda_{-} \delta\left(k_{i}-1, k_{i}^{\prime}\right) \delta\left(k_{j}-1, k_{j}^{\prime}\right) \\
& +\lambda_{+} \delta\left(k_{i}+1, k_{i}^{\prime}\right) \delta\left(k_{j}-1, k_{j}^{\prime}\right) \\
& +\lambda_{+} \delta\left(k_{i}-1, k_{i}^{\prime}\right) \delta\left(k_{j}+1, k_{j}^{\prime}\right) \\
& \left.+\lambda_{z}\left(k_{i}-1 / 2\right)\left(k_{j}-1 / 2\right) \delta\left(k_{i}, k_{i}^{\prime}\right) \delta\left(k_{j}, k_{j}^{\prime}\right)\right) .
\end{aligned}
$$

Los elementos de matriz no nulos de la Ecuación (5.5) conectan estados con $\Delta k=0$ y \pm 2 , donde $k=\sum_{i} k_{i}-N / 2$ es la proyección del espín total en la dirección $z$. En particular, para la elección $\lambda_{x}=\lambda_{y}=\lambda_{\perp}$, el operador $S_{z}=\sum_{i=1}^{N} s_{z, i}$ conmuta con el Hamiltoniano de la Ecuación (5.1) y los elementos de matriz se escriben

$$
\begin{aligned}
\left\langle m^{\prime}|H| m\right\rangle=\quad & \sum_{i \neq j=1}^{N} g(i, j)\left(\frac{\lambda_{\perp}}{2} \delta\left(k_{i}+1, k_{i}^{\prime}\right) \delta\left(k_{j}-1, k_{j}^{\prime}\right)\right. \\
& +\delta\left(k_{i}-1, k_{i}^{\prime}\right) \delta\left(k_{j}+1, k_{j}^{\prime}\right) \\
& \left.+\lambda_{z}\left(k_{i}-1 / 2\right)\left(k_{j}-1 / 2\right) \delta\left(k_{i}, k_{i}^{\prime}\right) \delta\left(k_{j}, k_{j}^{\prime}\right)\right) .
\end{aligned}
$$

Estos elementos son necesario para calcular exactamente los autovalores y autovectores, y con estos la correspondiente matriz densidad (Reboiro, 2008).

En la base de autovalores del Hamiltoniano (Ecuación (5.3)), construida como se comentó antes, se calculo la evolución temporal de los observables de espín utilizando 
el formalismo desarrollado en la Sección 5.2. El factor de Squeezing que se utilizó en este Capítulo para cuantificar la compresión en incerteza del espín atómico es el que se presento en la Sección 1.3.2, Ecuación (1.71). El estado inicial utilizado fue un estado de espín coherente como el descripto en la Sección 4.2.2.

\subsubsection{Algunos casos analíticamente solubles}

A continuación se tomará el caso $N=2$ átomos, y se considerarán distintas interacciones entre sitios de la cadena abierta.

\section{Interacción armónica}

Se escribe la interacción entre átomos con $g(i, j)=\sin \left(|i-j| \frac{\pi}{2}\right)$ y $\lambda_{z}=2 \lambda_{\perp}$. Esta elección de acoplamiento, en principio arbitraria, es utilizada por el hecho de que a menos que la simetría de la interacción (Ecuación (5.1)) esté rota, no habrá una ganancia neta en la compresión en incerteza de espín (Ecuación (1.71)), como se discutirá en la subsección siguiente. La diagonalización del Hamiltoniano nos devuelve los autovalores $e_{\alpha}$ y autovectores $\psi_{\alpha}(\operatorname{con} \alpha=1,2,3,4)$

$$
\begin{aligned}
\epsilon_{1}=\lambda_{\perp}, & \left|\psi_{1}\right\rangle=|0,0\rangle, \\
\epsilon_{2}=0, & \left|\psi_{2}\right\rangle=\frac{1}{2}(|1,0\rangle+|0,1\rangle), \\
\epsilon_{3}=-2 \lambda_{\perp}, & \left|\psi_{3}\right\rangle=\frac{1}{2}(|1,0\rangle-|0,1\rangle), \\
\epsilon_{4}=\lambda_{\perp}, & \left|\psi_{4}\right\rangle=|1,1\rangle .
\end{aligned}
$$

Con estos autovalores y autovectores se pueden calcular los observables de espín dependientes del tiempo, para diferentes orientaciones del estado coherente atómico como condición inicial del sistema. Se obtiene

$$
\begin{array}{r}
\left\langle S_{z}(t)\right\rangle=-\cos \theta_{0}, \\
\left\langle S_{z}^{2}(t)\right\rangle=\frac{1}{2}+\frac{1}{2} \cos ^{2} \theta_{0} .
\end{array}
$$

Estos cálculos pueden ser repetidos para diferentes orientaciones en el vector de condiciones iniciales, como: $\theta_{0}=\frac{\pi}{2}$ y $\phi_{0}=0$. Para esta elección el valor esperado de 
la componente $z$ total del espín se anula $\left\langle S_{z}\right\rangle=0$, y

$$
\begin{array}{r}
\langle\bar{S}\rangle=\left(\left\langle S_{x}\right\rangle, 0,0\right), \\
\left\langle S_{x}\right\rangle=-\cos \left(\frac{\lambda_{\perp} t}{\hbar}\right) .
\end{array}
$$

El vector unitario que define la dirección normal a $\langle\bar{S}\rangle$ es $\bar{n}=\left(0, \pm \sin \theta_{n}, \cos \theta_{n}\right)$.

El signo positivo corresponde a la elección $\phi_{n}=\frac{\pi}{2}$ y el signo menos a $\phi_{n}=\frac{3 \pi}{2}$. Con estos elementos, la expresión formal del factor de squeezing $\xi^{2}$ se escribe

$$
\xi^{2}=\frac{1 \pm 2 \sin \theta_{n} \cos \theta_{n} \sin \left(\frac{\lambda_{-} t}{\hbar}\right)}{\cos ^{2}\left(\frac{\lambda_{\perp} t}{\hbar}\right)} .
$$

Análogamente, la elección $\theta_{0}=0$ y $\phi_{0}=0$ da como resultado $\xi^{2}=1$ (no squeezing). El caso con $\theta_{0}=\frac{\pi}{2}, \phi_{0}=\frac{\pi}{2}$ es similar al caso $\theta_{0}=\frac{\pi}{2}, \phi_{0}=0$ y conduce a la misma expresión para $\xi^{2}$ que la Ecuación (5.10).

\section{Interacción Gausiana}

La interacción entre sitios de espines puede ser modelado por un factor de forma Gausiano

$$
g(i, j)=e^{-\frac{1}{2}(i-j)^{2}}
$$

La solución del problema de autovalores, para esta interacción se escribe

$$
\begin{array}{rlrl}
\epsilon_{1}=\frac{\lambda_{z}}{2} e^{-1 / 2}, & \left|\psi_{1}\right\rangle & =|0,0\rangle, \\
\epsilon_{2}=\left(\lambda_{\perp}-\frac{\lambda_{z}}{2}\right) e^{-1 / 2}, & \left|\psi_{2}\right\rangle & =\frac{1}{2}(|1,0\rangle+|0,1\rangle), \\
\epsilon_{3}=-\left(\lambda_{\perp}+\frac{\lambda_{z}}{2}\right) e^{-1 / 2}, & \left|\psi_{3}\right\rangle & =\frac{1}{2}(|1,0\rangle-|0,1\rangle), \\
\epsilon_{4} & =\frac{\lambda_{z}}{2} e^{-1 / 2}, & \left|\psi_{4}\right\rangle & =|1,1\rangle .
\end{array}
$$

Con la elección $\lambda_{z}=2 \lambda_{\perp}$ la expresión anterior se reduce a la solución de la Ecuación (5.7), pero con un factor $e^{-1 / 2}$, y para los ángulos de orientación $\theta_{0}=\frac{\pi}{2}$ y $\phi_{0}=0$ se obtiene

$$
\xi^{2}=\frac{1 \pm 2 \sin \theta_{n} \cos \theta_{n} \sin \left(\frac{\lambda_{-} t}{\hbar} e^{-1 / 2}\right)}{\cos ^{2}\left(\frac{\lambda_{\perp} t}{\hbar} e^{-1 / 2}\right)} .
$$


Y la misma expresión si se elige $\theta_{0}=\frac{\pi}{2}$ y $\phi_{0}=\frac{\pi}{2}$. Un análisis similar se puede realizar en el caso con $N=3$ átomos. La solución para ambas interacciones, es también analítica y conduce a resultados que son similares a los de las Ecuaciones (5.10 y 5.13).

\section{Casos de interacciones no-simétricas con $N=3$ átomos}

En esta subsección se discutirá, resolviendo analíticamente el caso de tres átomos, el efecto de la asimetría de la interacción sobre el factor de squeezing $\xi^{2}$. La idea principal detrás de esto es el mecanismo de ruptura de simetría inducido por las constantes de acoplamiento $\lambda_{i}$ de la Ecuación (5.3). En este ejemplo, la diagonalización del Hamiltoniano (Ecuación (5.3)) es realizada en la base de estados que resulta de la numeración de tres sitios (átomos) de la cadena con dos estados (posibles por sitio). Los autovalores de la ecuación secular son

$$
\begin{array}{r}
\epsilon_{1}=\epsilon_{8}=\frac{\lambda_{z}}{2}, \\
\epsilon_{2}=\epsilon_{5}=0, \\
\epsilon_{3}=\epsilon_{6}=-\frac{\lambda_{z}}{4}-\Delta, \\
\epsilon_{4}=\epsilon_{7}=-\frac{\lambda_{z}}{4}-\Delta,
\end{array}
$$

con

$$
\Delta=-\frac{\sqrt{\lambda_{z}^{2}+8 \lambda_{\perp}^{2}}}{4}
$$

La dependencia temporal de los valores medios puede ser calculados explícitamente y sus expresiones son

$$
\begin{aligned}
\left\langle S_{z}\right\rangle & =-\frac{3}{2} c_{0} \\
\left\langle S_{z}^{2}\right\rangle & =\frac{3}{4}+\frac{3}{2} c_{0}^{2} \\
\left\langle S_{+}^{2}\right\rangle & =e^{-2 i\left(\phi_{0}-\pi\right)} \frac{1}{2} s_{0}^{2}\left(A-i B c_{0}\right), \\
\left\langle S_{+}\right\rangle & =e^{-i\left(\phi_{0}-\pi\right)} \frac{1}{2} s_{0}\left[\frac{1}{2} s_{0}^{2} C+\left(1-\frac{1}{2} s_{0}^{2}\right) A-i B c_{0}\right], \\
\left\langle\left\{S_{+}, S_{-}\right\}\right\rangle & =3+s_{0}^{2} C,
\end{aligned}
$$




$$
\left\langle\left\{S_{+}, S_{z}\right\}\right\rangle=e^{-i\left(\phi_{0}-\pi\right)} \frac{1}{2} s_{0}\left[-c_{0} A+i\left(1-\frac{1}{2} s_{0}^{2}\right) B\right]
$$

y

$$
\begin{aligned}
\left\langle S_{x}\right\rangle & =\operatorname{Re}\left\langle S_{+}\right\rangle, \\
\left\langle S_{y}\right\rangle & =\operatorname{Im}\left\langle S_{+}\right\rangle, \\
\left\langle S_{x}^{2}\right\rangle & =\frac{1}{2} \operatorname{Re}\left\langle S_{+}^{2}\right\rangle+\frac{1}{4}\left\langle\left\{S_{+}, S_{-}\right\}\right\rangle, \\
\left\langle S_{y}^{2}\right\rangle & =-\frac{1}{2} \operatorname{Re}\left\langle S_{+}^{2}\right\rangle+\frac{1}{4}\left\langle\left\{S_{+}, S_{-}\right\}\right\rangle,
\end{aligned}
$$

con

$$
\begin{aligned}
& c_{0}=\cos \theta_{0}, \\
& s_{0}=\sin \theta_{0},
\end{aligned}
$$

en correspondencia con la definición de los ángulos de orientación del estado coherente de espín Ecuación (1.38). Las definiciones de las nuevas cantidades introducidas en los valores medios de espín son:

$$
\begin{aligned}
A= & {\left[\frac{3}{2}+\frac{1}{2}(\alpha-2 \beta)\right] \cos \left(\Delta+\frac{3}{4} \lambda_{z} t\right), } \\
& +\left[\frac{3}{2}-\frac{1}{2}(\alpha-2 \beta)\right] \cos \left(\Delta-\frac{3}{4} \lambda_{z} t\right), \\
B= & {\left[\frac{3}{2}+\frac{1}{2}(\alpha-2 \beta)\right] \sin \left(\Delta+\frac{3}{4} \lambda_{z} t\right), } \\
& -\left[\frac{3}{2}-\frac{1}{2}(\alpha-2 \beta)\right] \sin \left(\Delta-\frac{3}{4} \lambda_{z} t\right), \\
C= & 3-(\alpha+\beta)^{2} \sin ^{2}(\Delta t),
\end{aligned}
$$

con

$$
\begin{aligned}
\alpha & =\frac{\lambda_{z}-4 \lambda_{\perp}}{4 \Delta}, \\
\beta & =\frac{\lambda_{z}+2 \lambda_{\perp}}{4 \Delta}, \\
3 & =\alpha^{2}+2 \beta^{2} .
\end{aligned}
$$

Entonces es fácil mostrar que para una interacción simétrica $\left(\lambda_{z}=\lambda \perp\right)$ se obtiene

$$
\Delta=\frac{3}{4} \Lambda_{z}
$$




$$
\begin{aligned}
& \alpha=-1, \\
& \beta=1, \\
& A=3, \\
& B=0 \\
& C=3 .
\end{aligned}
$$

Con estos resultados, los valores medios se escriben como

$$
\begin{aligned}
\left\langle S_{z}\right\rangle & =-\frac{3}{2} c_{0}, \\
\left\langle S_{z}^{2}\right\rangle & =\frac{3}{4}+\frac{3}{2} c_{0}^{2}, \\
\left\langle S_{+}^{2}\right\rangle & =e^{-2 i\left(\phi_{0}-\pi\right)} \frac{3}{2} s_{0}^{2}, \\
\left\langle S_{+}\right\rangle & =e^{-i\left(\phi_{0}-\pi\right)} \frac{3}{2} s_{0}, \\
\left\langle\left\{S_{+}, S_{-}\right\}\right\rangle & =3\left(1+s_{0}^{2}\right), \\
\left\langle\left\{S_{+}, S_{z}\right\}\right\rangle & =-3 e^{-i\left(\phi_{0}-\pi\right)} s_{0} c_{0} .
\end{aligned}
$$

La dirección del espín total se determina por las relaciones

$$
\begin{aligned}
& \frac{\left\langle S_{x}\right\rangle}{\langle|\bar{S}|\rangle}=-s_{0} \cos \phi_{o} . \\
& \frac{\left\langle S_{z}\right\rangle}{\langle|\bar{S}|\rangle}=s_{0} \sin \phi_{0} . \\
& \frac{\left\langle S_{z}\right\rangle}{\langle|\bar{S}|\rangle}=-c_{0} .
\end{aligned}
$$

Por lo tanto, a partir de estas relaciones se obtiene

$$
\begin{aligned}
\left(\Delta S_{n}\right)^{2} & =\frac{3}{4} \\
|\bar{S}| & =\frac{3}{4},
\end{aligned}
$$

y en consecuencia

$$
\xi^{2}=N \frac{\left(\Delta S_{n}\right)^{2}}{|\bar{S}|}=1
$$

Una observación interesante de este resultados es que no depende del valor del ángulo $\phi_{0}$. Respecto a la simetría, este resultado nos indica que para obtener valores $\xi^{2}<1$, 
la simetría del Hamiltoniano de la Ecuación (5.1) debe estar rota (ver también (Reboiro, 2008; Civitarese and Reboiro, 2006; Reboiro et al., 2007; Civitarese et al., 2009)). Más adelante utilizaremos las Ecuaciones (5.10 y 5.13) para ganar cierta información acerca de los casos que no son solubles analíticamente.

\section{Aproximacióon TDA para gran número de átomos}

La Aproximación Tamm-Dancoff (TDA), puede utilizarse para calcular observables en el límite de gran número de átomos. La aproximación TDA está basada en la expansión bosónica de los operadores de espín y un posterior mapeo en la base de un fonón. Esta se realiza aplicando los siguientes pasos (Klein and Marshalek, 1991):

a) Expansión bosónica de los operadores de espín: Los operadores son expresados en término de los operadores bosónicos asociados a cada sitio de la cadena, esto es,

$$
\begin{array}{r}
S_{+}=\sum_{i} b_{i}^{+}, \\
S_{-}=\sum_{i} b_{i}, \\
S_{z}=\sum_{i} b_{i}^{+} b_{i}-\frac{N}{2} .
\end{array}
$$

El estado de vacío $|0\rangle$ se define como el estado con todos los átomos en su nivel más bajo, de tal manera que los operadores bosónicos de bajada del $i$-ésimo sitio, cumplan $b_{i}|0\rangle=0$. Claramente los operadores de la Ecuación (5.23) obedecen el álgebra de $S U(2)$.

b) Base de un fonón: Los operadores bosónicos $b_{i}^{+}$(donde el subíndice- $i$ indica el sitio) puede transformarse en una nueva base de los operadores bosónicos $\Gamma_{n}^{\dagger}, \mathrm{y} \Gamma_{n}$, que son una combinación lineal de la forma

$$
\Gamma_{n}^{+}=\sum_{i} X_{n}(i) b_{i}^{+}
$$

Los nuevos operadores actúan sobre el mismo vacío $|0\rangle$, así

$$
\Gamma_{n}|0\rangle=0
$$


y el conjunto de estados generados por estos se escriben

$$
|n\rangle=\Gamma_{n}^{+}|0\rangle
$$

Si se invierte la Ecuación (5.24), a través de la transformación inversa se obtiene

$$
b_{i}^{+}=\sum_{n} X_{n}^{*}(i) \Gamma_{n}^{+}
$$

ya que la transformación definida en la Ecuación (5.24) preserva las relaciones de conmutación de los operadores bosónicos originales.

c) Diagonalización TDA: El Hamiltoniano de la Ecuación (5.3) se transforma a la base de bosones $b_{i}^{+}\left(\mathrm{y} \quad b_{i}\right)$ y posteriormente a la base de operadores de un fonón (Ecuación (5.24)). La imagen TDA del Hamiltoniano se escribe

$$
\begin{aligned}
H_{T D A} & =\sum_{i} \epsilon_{i}\left(\hat{n}_{i}-\frac{1}{4}\right)+\sum_{i \neq j} \lambda_{\perp} g_{i j} b_{i}^{+} b_{j}, \\
& =\sum_{n} E_{n} \Gamma_{n}^{+} \Gamma_{n},
\end{aligned}
$$

con

$$
\epsilon_{i} \sum_{j \neq i} \lambda_{z} g_{i j}
$$

El espíritu de la transformación TDA (Klein and Marshalek, 1991) es la de linealizar la ecuación de movimiento

$$
\left[H_{T D A}, \Gamma_{m}^{+}\right]=E_{n} \Gamma_{n}^{+}
$$

que conduce a la ecuación secular

$$
\epsilon_{l} X_{n}(l)+\sum_{k \neq l} \lambda_{\perp} g_{l k} X_{n}(k)=E_{n} X_{n}(l)
$$

Por lo tanto, sustituyendo la amplitud

$$
X_{n}(l)=\frac{1}{E_{n}-\epsilon_{l}} \sum_{k \neq l} \lambda_{\perp} g_{l k} X_{n}(k)=\frac{\Lambda_{n}(l)}{E_{n}-\epsilon_{l}},
$$

en la Ecuación (5.31) se obtiene la relación de dispersión para las energías $E_{n}$ en la aproximación TDA. Las amplitudes TDA se normalizan mediante

$$
\sum_{l}\left|X_{n}(l)\right|^{2}=1
$$


d) Condición inicial: Una vez realizada la transformación TDA bosónica, la condición inicial puede ser escrita en términos de fonones TDA

$$
|I\rangle=\mathcal{N} e^{\left(z \sum_{n=1}^{N} \lambda_{n} \Gamma_{n}^{\dagger}\right)}|0\rangle,
$$

donde $\mathcal{N}$ es la norma del estado $|I\rangle$.

e) Los operadores de espín en la base TDA: Como se hizo anteriormente para la condición inicial y el Hamiltoniano, los operadores de espín pueden ser reescritos en la base TDA:

$$
\begin{array}{r}
S_{z}=\sum_{n} \Gamma_{n}^{\dagger} \Gamma_{n}-\frac{N}{2}, \\
S_{+}=\sum_{n} \lambda_{n} \Gamma_{n}^{\dagger},
\end{array}
$$

con

$$
\lambda_{n}=\sum_{k} X_{n}(k)
$$

El factor de squeezing puede ser fácilmente obtenido, luego de realizar las operaciones algebraicas para obtener

$$
\begin{aligned}
\left\langle S_{z}\right\rangle & =-\frac{N}{2}+\frac{|z|^{2} N}{1+|z|^{2} N}, \\
\left\langle S_{z}^{2}\right\rangle & =\frac{N^{2}}{4}-(N-1) \frac{|z|^{2} N}{1+|z|^{2} N}, \\
\left\langle S_{+}\right\rangle & =\frac{z^{*}}{1+|z|^{2} N} \sum_{n=1}^{N}\left|\lambda_{n}\right|^{2} e^{i\left(E_{n}-E_{0}\right) t}, \\
\left\langle S_{+}^{2}\right\rangle & =0 \\
\left\langle\left\{S_{+}, S_{-}\right\}\right\rangle & =N+\frac{2|z|^{2}}{1+|z|^{2} N} \sum_{n=1}^{N}\left|\lambda_{n}\right|^{4}, \\
\left\langle\left\{S_{+}, S_{z}\right\}\right\rangle & =-(N-1)\left\langle S_{+},\right\rangle
\end{aligned}
$$

Para los valores esperados de espín, en el límite de $N$ grande se obtiene

$$
\begin{aligned}
\left\langle S_{z}\right\rangle & =-\frac{N}{2}, \\
\left\langle S_{z}^{2}\right\rangle & =\frac{N^{2}}{4} \\
\left\langle S_{+}\right\rangle & =\left\langle S_{+}^{2}\right\rangle=0,
\end{aligned}
$$




$$
\begin{aligned}
\left\langle\left\{S_{+}, S_{-}\right\}\right\rangle & =N, \\
\left\langle\left\{S_{+}, S_{z}\right\}\right\rangle & =0,
\end{aligned}
$$

por lo tanto, reemplazando estas expresiones en la definición del factor de squeezing de la Ecuación (1.3.2) se obtiene

$$
\xi_{\text {Ngrande }}^{2} \rightarrow \frac{\left(N^{2} / 4\right)|\bar{n}|^{2}}{\left(N^{2} / 4\right)}=1,
$$

ya que $\bar{n}$ es un vector unitario. El resultado anterior parece indicar que, al menos en el subespacio TDA, el factor de squeezing satura para un gran número de átomos. Naturalmente para una prueba completa de este límite se necesita diagonalizar sistemas con un gran número de átomos. Pero esta tarea es limitada por el crecimiento exponencial $\left(2^{N}\right)$ de la dimensión del espacio a diagonalizar, cuando $N$ se incrementa.

\subsection{Resultados y discusión}

En esta sección se presentará la discusión de los resultados provenientes del formalismo discutido en la Sección anterior, para el factor de squeezing $\xi^{2}$, para varias interacciones en los sitios de espín. En todos los casos se consideraron átomos de dos niveles, con un espín $s=1 / 2$ en cada sitio. Los factores $g(i, j)$ de las interacciones que se diagonalizaron para $N \leq 11$, son los siguientes

$$
\begin{aligned}
& g(i, j)=\left|\sin \left(\frac{\pi}{2}(i-j)\right)\right|, \quad(a) \\
& g(i, j)=\sin \left(\frac{\pi}{2}(i-j)\right), \quad(b) \\
& g(i, j)=e^{-\frac{1}{2}(i-j)^{2}}, \quad(c) \\
& g(i, j)=\left(\sin \left(\frac{\pi}{N}(i-j)\right)\right) \cdot \cdot^{-2}(d)
\end{aligned}
$$

Estas interacciones son periódicas y de largo alcance, y han sido discutida en la literatura (Shastry, 1988; Haldane, 1988; Inozemtsev and Inozemtseva, 1991; Inozemtsev and Kuzemsky, 1991; Inozemtsev, 1995; Frahm and Inozemtsev, 1994; Inozemtsev and Dorfel, 1993; Inozemtseva and Inozemtsev, 1997; Dittrich and Inozemtsev, 1997). 
Las constantes $\lambda_{\perp}$ y $\lambda_{z}$ de la Ecuación (5.3), están fijos en los valores $\lambda_{\perp}=1$ y $\lambda_{z}=2$, para todos los casos, para forzar la asimetría de las interacciones (Reboiro et al., 2007)(Reboiro, 2008)(Civitarese and Reboiro, 2006), como se explico en capítulos anteriores. Como condición inicial se tomó un estado (Ecuación (1.38)) $\operatorname{con} \theta_{0}=\pi / 2, \pi / 4$ y $\pi / 8$, y $\phi_{0}=0$.

En las Figuras 5.1-5.2 se muestra la dependencia temporal del parámetro de squeezing $\xi^{2}$ para una cadena con $N=5$ átomos. El procedimiento consistió en: (i) diagonalización del Hamiltoniano de la (Ecuación (5.3)) en la base escrita en la Ecuación(5.4); (ii) cálculo de los correspondientes autovectores y autovalores, que se utilizan para construir la matriz densidad dependiente del tiempo Ecuaciones (2.14);(iii) cálculo de los de los valores medios de las componentes de espín que entran en la definición del parámetro de squeezing (Ecuación (1.71))

La Figura 5.1 muestra los resultados obtenidos para diferentes $g(i, j)$ de las Ecuaciones (5.39) y para diferentes condiciones iniciales del estado coherente con $\theta_{0}=\pi / 2$ y $\phi_{0}=0$. Aunque, para la mayoría de los casos los resultados del squeezing se encuentran cerca del límite canónico $\left(\xi^{2}=1 / 2\right)$, uno puede ver rápidas oscilaciones de los valores reales. Esto significa que los valores medios son mucho mas grandes que el límite canónico. Este comportamiento es fácilmente entendido debido a que para $\theta_{0}=\pi / 2$, el espín medio es perpendicular a la dirección $z$. Para investigar la dependencia temporal de $\xi^{2}$ sobre las condiciones iniciales se han repetido los cálculos para $\theta_{0}=\pi / 4$ y $\phi_{0}=\pi / 8$. Los resultados se muestran en las Figuras 5.2 y 5.3 , respectivamente. Se observa que para valores pequeños de $\theta_{0}$, las oscilaciones de $\xi^{2}$ están confinadas en una región relativamente estrecha $0,7<\xi^{2}<1$ ver Figura 5.3. Para el caso límite $\theta_{0}=0$ se obtiene $\xi^{2}=1$, como era de esperar. De estos resultados, puede concluirse que la dependencia temporal de $\xi^{2}$ puede se controlada por la interacción espín-espín, como también por la elección del estado coherente inicial. A primera vista, una elección apropiada del estado coherente puede, en efecto minimizar el tiempo medio del valor de $\xi^{2}$. Los valores óptimos de $\xi^{2}$ son del orden de 0,7 . De los resultados mostrados en la Figuras 5.1-5.3, uno puede concluir que una cadena de espines con interacciones espín-espín modulada por factores $g(i, j)$, como los de la 
Ecuación (5.39), puede ser considerada como un dispositivo eficaz para mantener información sobre la orientación del espín y la relativa intensidad de las componentes del espín total. Para realizar una comparación se muestran, en la Figura 5.4, los resultados correspondientes a las Ecuaciones (5.10 y 5.13). Mientras que los resultados obtenidos para $g(i, j)=\sin \left|\frac{\pi}{2}(i-j)\right|(\operatorname{caso}(\mathrm{a}))$ muestra oscilaciones alrededor del mínimo a $\xi^{2}=1 / 2$, los obtenidos con $g(i, j)=e^{-\frac{1}{2}(i-j)^{2}}$ (caso (b)) muestran, además, una modulación grande como en los casos previos. Una comparación entre las curvas mostradas en las Figuras 5.1(b) y 5.1(c) indican que incrementando el número de átomos, las oscilaciones son más rápidas y el valor mínimo de $\xi^{2}$ se incrementa significativamente

Ahora se comentará la dependencia del parámetro de squeezing con $N$. Las Figuras 5.5-5.6 muestran los resultados del parámetro de squeezing $\xi^{2}$ como función del número de sitios de espín. Los resultados en la Figura 5.5 son mínimos locales, esto es, que cada uno de los puntos muestran el menor valor de $\xi^{2}$ calculados a un valor fijo de $N$ y en el mismo intervalo temporal de las Figuras 5.5 son mínimos locales, esto es que los puntos en las figuras son los menores valores de $\xi^{2}$ que se obtienen a $N$ fijo en el mismo intervalo temporal de las Figuras 5.1-5.3. Los resultados que se muestran en la Figura 5.6 son los valores medios en el intervalo temporal, para las mismas interacciones. Para calcular los promedios se incrementó el intervalo temporal de las Figuras 5.1-5.3 a un valor máximo de $t=1000$. Como característica general se observó que, relativamente, los mínimos locales y el valor promedio en $\xi^{2}$ se obtienen con la interacción $g(i, j)=\left(\sin \left(\frac{\pi}{N}(i-j)\right)\right)^{-2}$ (Shastry, 1988; Haldane, 1988; Inozemtsev and Kuzemsky, 1991). Esta interacción que representa un intercambio $1 / r^{2}$, dada una distancia $r$ entre sitios, fue propuesta por (Shastry, 1988) y por (Haldane, 1988), en el estudio de una cadena isotrópica de Heisenberg antiferromagnética de espín-1/2. Posteriormente, el espectro de una cadena isotrópica de Heisenberg fue resuelto exactamente utilizando la misma interacción de largo alcance (Inozemtsev and Kuzemsky, 1991). Considerando el significado físico de las interacciones de Shastry (1988) y Haldane(1988), los resultados que se presentan parecen (aunque no en forma concluyente) mostrar que las interacciones de largo 
alcance pueden optimizar los dispositivos de espín-squeezing.

\subsection{Conclusiones del capítulo}

Para resumir, en este capítulo se mostraron resultados de haber calculado el factor de squeezing, $\xi^{2}$, para cadenas abiertas de pseudo-espín $s-1 / 2$ con interacciones periódicas de largo alcance. Se investigó la dependencia de $\xi^{2}$ con: (i) las interacciones,(ii) el número de átomos, y (iii) las condiciones iniciales. Los resultados, en general, son dependientes de las condiciones iniciales que en este capítulo han sido representados por estados coherentes de espín. Aunque el número de átomos incluidos en los cálculo es relativamente bajo $(N \leq 11)$ el tamaño del espacio de configuraciones es lo suficientemente grande como para ver una tendencia de los resultados. Para la situación con un gran número de átomos se discutió el uso de la aproximación TDA, que es exacta en el subespacio de pares de espín. Para este subespacio, los resultados sugieren saturación de $\xi^{2}$. Con estas limitaciones en mente, uno puede concluir diciendo que las cadenas de pseudo-espín con interacciones de largo alcance pueden ser dispositivos de espín óptimos en lo concerniente a la persistencia del factor de squeezing $\xi^{2}$. 


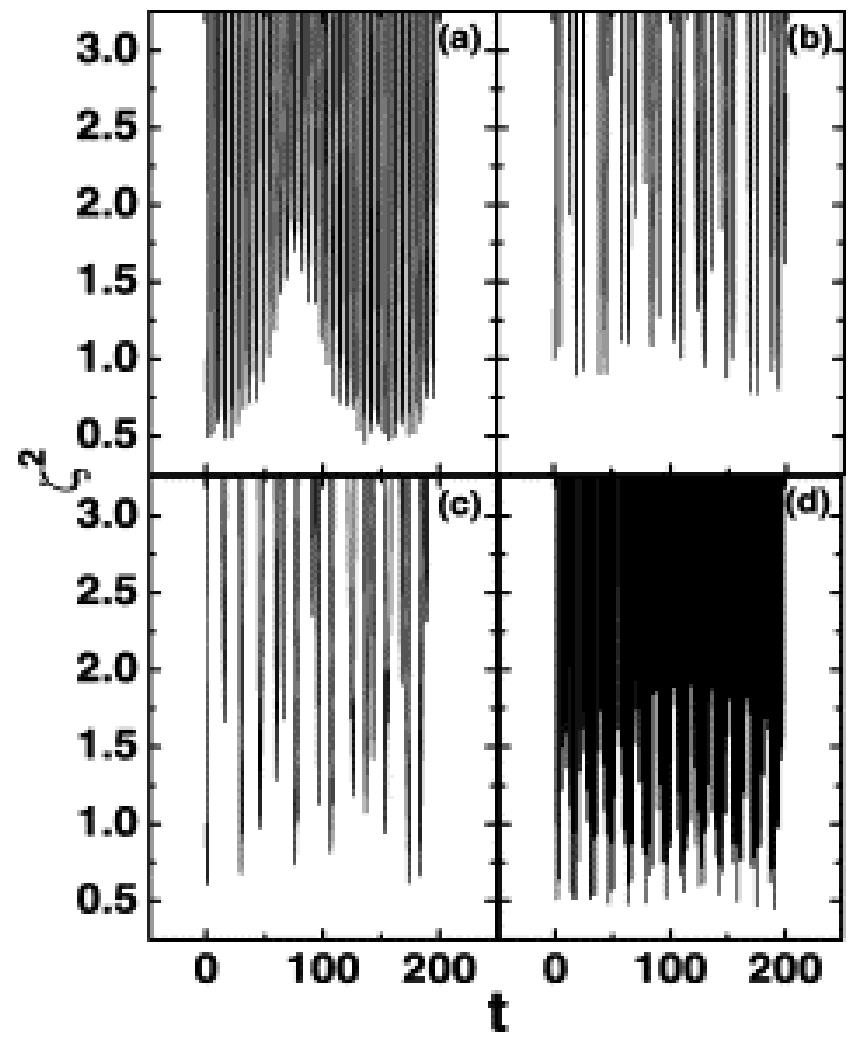

Figura 5.1: Dependencia temporal del parámetro de squeezing $\xi^{2}$, para diferentes interacciones. Los gráficos (a)-(d) muestran los resultados correspondientes a $g(i, j)$ de la Ecuación (5.39). Las constantes $\lambda_{\perp}$ y $\lambda_{z}$ se fijaron en los valores $\lambda_{\perp}=1$ y $\lambda_{z}=2$. El estado inicial, es un estado coherente (Ecuación (1.38)) con $\theta_{0}=\pi / 2$ y $\phi_{0}=0$. El tiempo está dado en unidades arbitrarias. Los resultados se corresponden al caso $N=5$ átomos. 


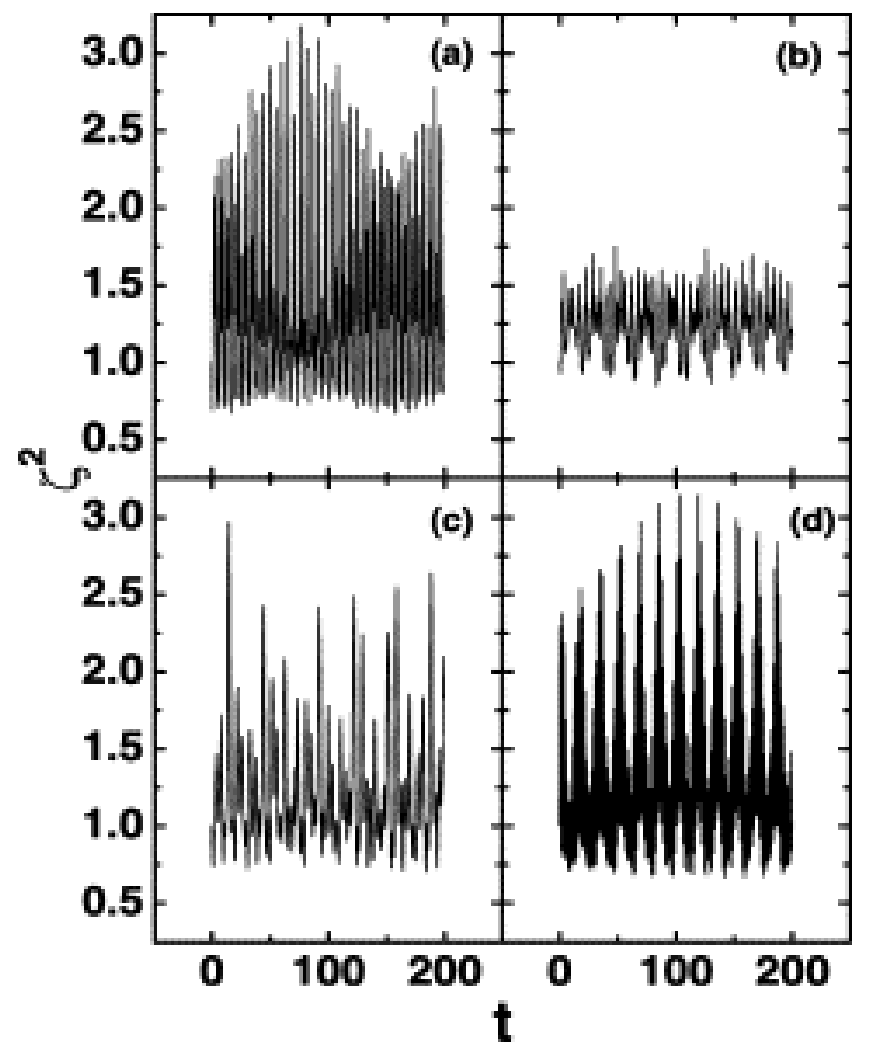

Figura 5.2: Idem Figura5.1, para un estado coherente con $\theta_{0}=\pi / 4$ y $\phi_{0}=0$ 


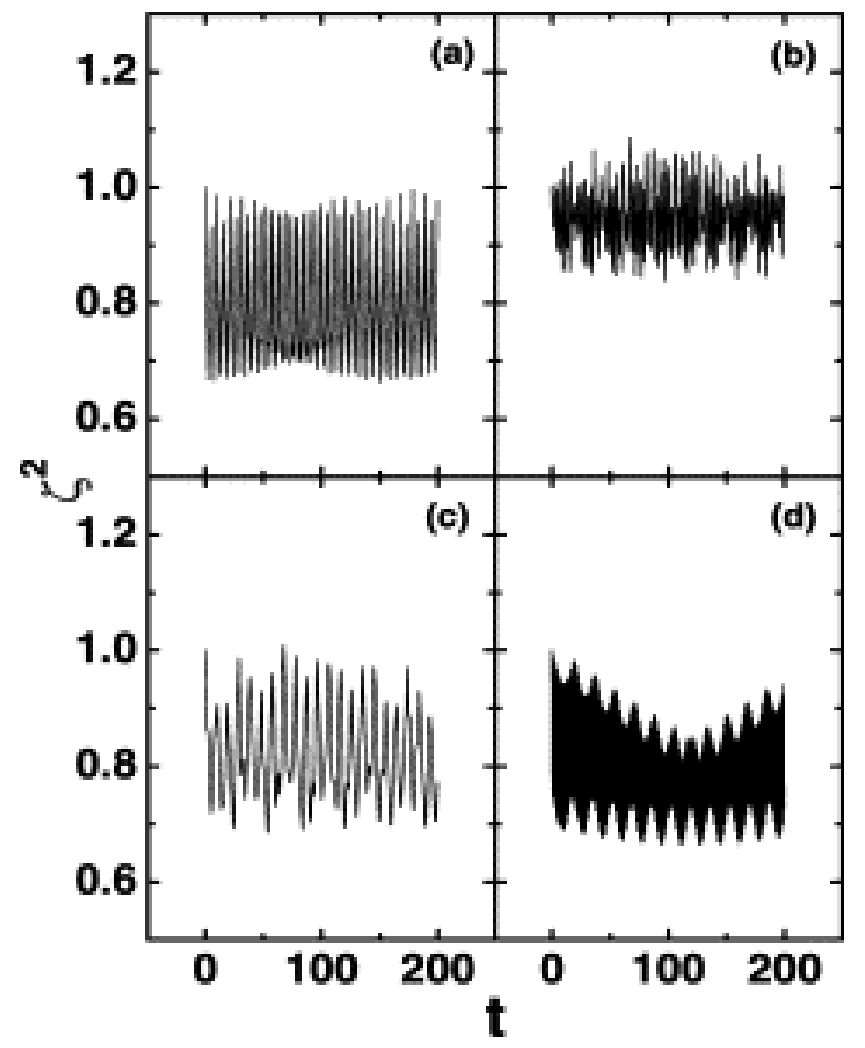

Figura 5.3: Idem Figura5.1, para un estado coherente (Ecuación (1.38)) con $\theta_{0}=\pi / 8$ y $\phi_{0}=0$ 


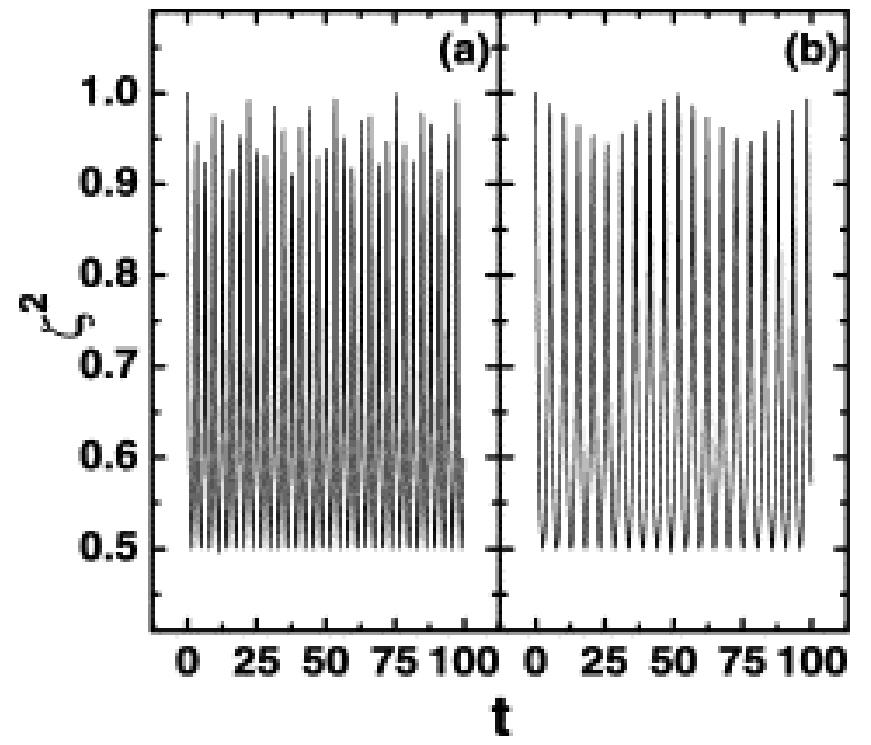

Figura 5.4: Parámetro de squeezing $\xi^{2}$, de la Ecuación (5.10) (gráfico (a)) y de la Ecuación (5.13) (gráfico (b)). Las curvas se obtuvieron con los autovectores y autovalores de las ecuaciones 5.7 y 5.12 respectivamente, para $N=2$. El estado inicial es un estado coherente (Ecuación (1.38)) con $\theta_{0}=\pi / 2$ y $\phi_{0}=0$. 


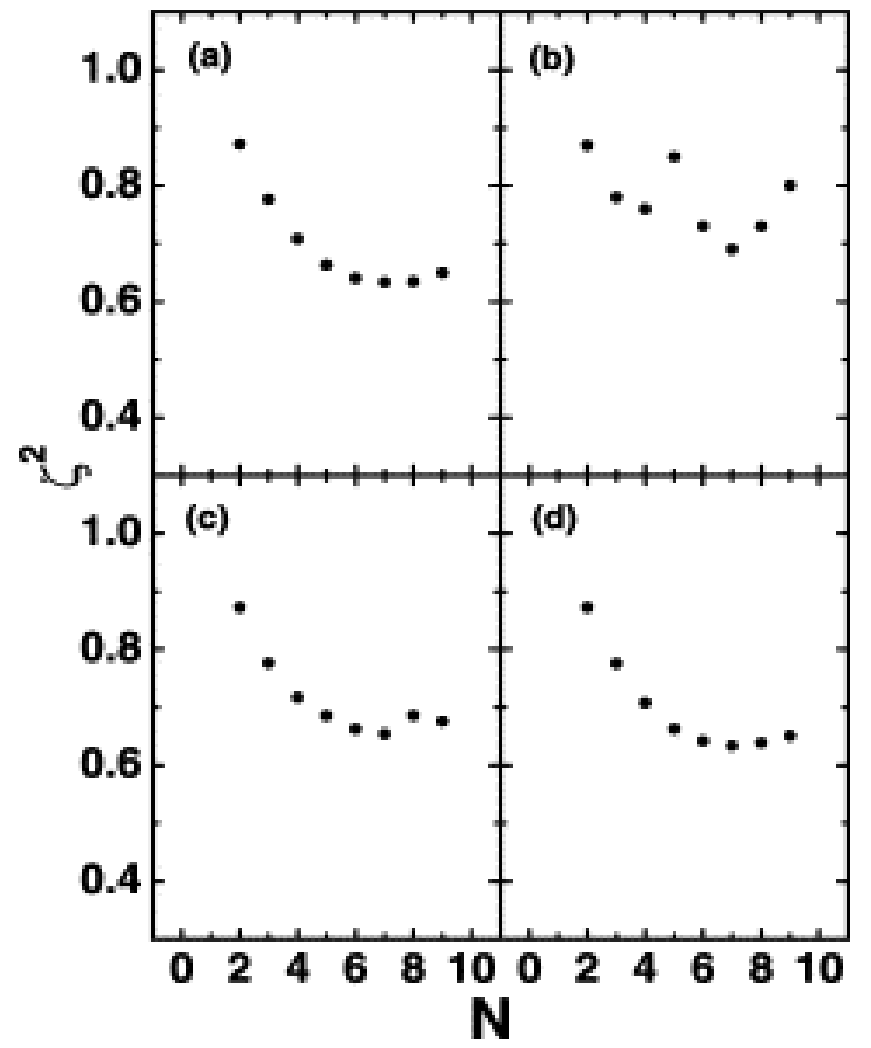

Figura 5.5: Valor mínimo del factor de squeezing $\xi^{2}$, como función del número de átomos $N$, para diferentes interacciones. En los gráficos (a)-(d) se muestran los resultados para la interacciones de la Ecuación (5.39). Se eligieron $\lambda_{\perp}=1, \lambda_{z}=2$, y un estado inicial coherente (Ecuación (1.38)) con $\theta_{0}=\pi / 8$ y $\phi_{0}=0$. Como se explica en el texto, para encontrar el mínimo, se estudió la evolución de $\xi^{2}$ en el intervalo $0<t<200$ 


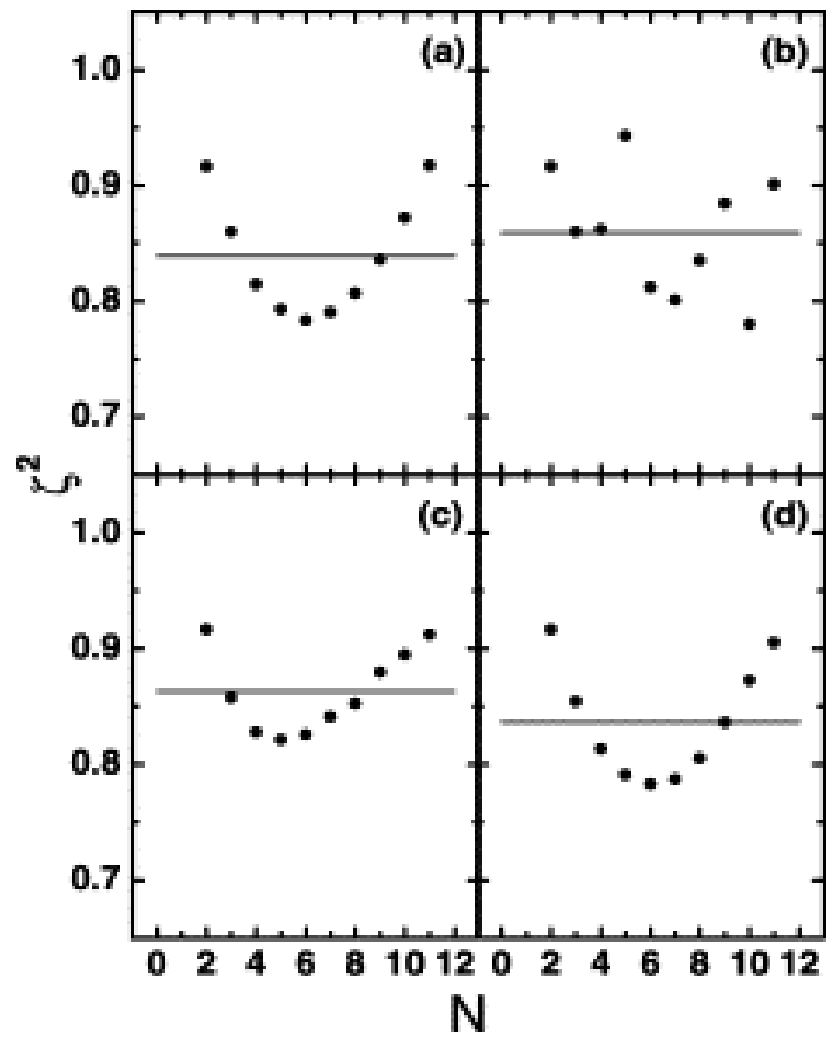

Figura 5.6: Promedio (puntos) y sus valor medio (línea), del factor de squeezing $\xi^{2}$ en función del número de átomos, y para cada una de las interacciones discutidas en este capítulo. Los parámetros utilizados para los cálculos están dados en las referencias de la Figura 5.5 


\section{Conclusiones}

En esta tesis se estudiaron los estados comprimidos de espín en sistemas espínbosones, formados por niveles atómicos (espines) y campos de radiación (fotones) que son de interés en computación e información cuántica. En particular se estudiaron sistemas a temperatura cero, cuyos Hamiltonianos corresponden a sistemas realizables experimentalmente con átomos fríos y luz coherente.

En el Capítulo 2 se estudió la dependencia de la compresión en incerteza del espín atómico en átomos de tres niveles, en una configuración escalera $(\Xi)$, que se encuentra en resonancia con un campo de radiación. Se introdujo el modelo algebraico utilizado y se mostraron los detalles para construir los observables de espín en función del tiempo. Se estudió la dependencia de las soluciones respecto de las parametrizaciones de las constantes del modelo y las condiciones iniciales. Se determinó que:

a) Si se utiliza un estado número como condición inicial del sistema, en el sector fotónico, no se obtiene compresión en la incerteza del espín atómico. Por otra parte, la utilización de estados coherentes, en dicho sector, fue una condición necesaria para la aparición de estados comprimidos de espín;

b) La transferencia de espín entre átomos y fotones es favorecida si la interacción, entre los niveles atómicos y los fotones, es parametrizados en una forma no simétrica. Esto se logra tomando $g_{2}>g_{1}$ en el Hamiltoniano del sistema (Ecuación (2.1)), ya que, para el caso $g_{1} \geq g_{2}$ no ocurre. También se encontró que la utilización de un estado coherente no conduce, en forma automática, a la compresión de la incerteza de espín, a menos que la interacción considerada sea no-simétrica en la forma antes explicada; 
c) Para una dada parametrización se encuentra que al aumentar el número de átomos se borra la compresión en la incerteza de espín.

En el capítulo 3 se extendió el modelo considerado y estudiamos un sistema de átomos de tres niveles en una cavidad que posee dos modos, en las configuraciones $\Xi, \Lambda$ y $V$. Para modelar las interacciones átomo-átomo se incluyeron interacciones efectivas dipolo-dipolo, y se estudió compresión de la incerteza del espín atómico en base a la asimetría de las constantes de acoplamiento en la interacción átomo-fotón del Hamiltoniano propuesto, así como también por la inclusión del término de interacción dipolo-dipolo. Se tomó el espaciamiento de niveles atómicos que corresponden al ${ }^{87} R b$. Para las configuraciones estudiadas, se encontró que la aparición de estados comprimidos de espín depende de varias condiciones físicas. En primera instancia, se puede decir que para las configuraciones $\Lambda$ y $\Xi$ la ocurrencia de compresión en incerteza de espín es evidente. Por otro lado para la configuración $V$ la compresión en incerteza del espín atómico no aparece tan claramente, si exploramos los valores de las constantes de acoplamiento, número de átomos y fotones. Las características más evidentes provienen de las configuraciones $\Lambda$ y $\Xi$ aparentemente indicando que, en ausencia de interacciones dipolo-dipolo, las variaciones en el número de átomos no afectan los observables de espín. En la misma condición $(g=0)$ el incremento en el número de fotones tiende a favorecer la aparición de estados comprimidos de espín en la configuración $\Xi$, y apenas modifica la evolución temporal de los observables de espín en la configuración $\Lambda$. Respecto al efecto de la interacción dipolar, se puede decir que esta no afecta a la compresión de la incerteza del espín atómico en la configuración $\Xi$, pero se vuelve crucial destruyendo los patrones en la configuración $\Lambda$.

En el capítulo 4 se estudió la transferencia de espín entre fotones y niveles atómicos localizados en una cadena de átomos de dos niveles. El Hamiltoniano del sistema se modeló por un término de radiación, por interacciones átomo-átomo y átomoradiación. Se modelaron efectos disipativos a través de interacciones de intercambio dadas por un término átomo-fotón con constante de acoplamiento compleja. Los resultados, en relación con los observables de espín para este sistema, se puede resumir 
como:

a) La inclusión de la interacción átomo-átomo produce la mejora de la compresión en la incerteza del espín atómico;

b) La presencia del término de disipación limita la transferencia de espín del campo de radiación a los niveles atómicos;

c) El estado inicial atómico, en nuestro caso, un estado de espín coherente (CSS), es tan importante como el estado inicial del campo de radiación (también un estado coherente) en la producción de estados comprimidos de espín;

d) El principal efecto entre las interacciones átomos-fotón y átomo-átomo es que ellos agregan incoherencia a pesar del número de fotones involucrado;

e) La dependencia de la transferencia de espín sobre los acoplamientos atómicos es evidente y abre la posibilidad modelar los efectos de la transferencia de espín por medio de redes atómicas, y/o trampas ópticas.

En el capítulo 5 se estudió numérica y analíticamente la dependencia de la compresión en incerteza del espín atómico para cadenas abiertas de átomos de dos niveles con interacciones periódicas dependientes del sitio. En particular se utilizó la aproximación TDA para estudiar la dependencia de la compresión en incerteza del espín con el número de átomos utilizados en la cadena.

Los resultados, en general, son dependientes de las condiciones iniciales y se han utilizado estados coherentes de espín para representarlas. Para la situación con un gran número de átomos se discutió el uso de la aproximación TDA, que es exacta en el subespacio de pares de espín. Se encontró que para este subespacio, los resultados sugieren saturación de $\xi^{2}$. Con estas limitaciones en mente, uno puede concluir diciendo que las cadenas de espines atómicos con interacciones de largo alcance son candidatos como dispositivos que presentan persistencia en la compresión del espín atómico.

Como resumen de los sistemas estudiados se puede decir que los resultados, en 
general, son fuertemente dependientes de las condiciones iniciales utilizadas y los valores de las constantes de acoplamiento. En los sistemas en interacción con un campo de radiación, la utilización de estados coherentes para la parte fotónica de los sistemas resultó una condición necesaria para la aparición de estados comprimidos de espín atómico. La utilización de estados coherentes de espín como condición inicial en la parte atómica, y una selección adecuada de la orientación en la esfera de Bloch, favorece la aparición de compresión en la incerteza de espín en los sistemas de dos niveles.

Como era de esperarse, el efecto de la interacción disipativa que se estudió en las cadenas de espínes "elimina” la compresión de la incerteza del espín atómico. Al considerarse interacciones dipolo-dipolo entre átomos de tres niveles, se observó que estas tienden a borrar la compresión de la incerteza de espín atómico para la configuración $\lambda$ y prácticamente no tienen efecto en la compresión de incerteza de la configuración $\Xi$. En las cadenas de espines con interacciones de largo alcance, uno de los resultados, más interesante que se obtuvo fue para el límite de gran número de átomos, analizando el sistema en la aproximación TDA, ya que se ve una saturación en el parámetro de squeezing.

Los resultados que se obtuvieron en el desarrollo de esta tesis han renovado la perspectiva que teníamos originalmente sobre el problema y dejan nuevas inquietudes que serán motivo de futuros trabajos, algunas de ellas son: (i) El estudio de la transferencia de correlaciones entre el campo de radiación y de los átomos dependiendo de los estados iniciales del campo electromagnético (Estados térmicos, comprimidos en incerteza, etc) y los estados iniciales de los sistemas atómicos; (ii) la conexión entre los estados comprimidos de los sistemas estudiados con el entrelazamiento cuántico; (iii) y finalmente, la utilización de otras técnicas (RPA, bosonizaciones, funciones de correlación de pares de espines, etc) para el estudio de la compresión en incerteza para sistemas con gran número de átomos. 


\section{Apéndice A}

\section{Cuantificación del campo electromagnético}

Como punto de partida, para la cuantización del campo electromagnético, se toman las ecuaciones clásicas del campo. En ausencia de fuentes, las Ecuaciones de Maxwell se expresan como

$$
\begin{aligned}
\nabla \cdot \mathbf{B} & =0 \\
\nabla \times \mathbf{E} & =-\frac{\partial \mathbf{B}}{\partial t}, \\
\nabla \cdot \mathbf{D} & =0 \\
\nabla \times \mathbf{H} & =\frac{\partial \mathbf{D}}{\partial t} .
\end{aligned}
$$

Donde el campo $\mathbf{B}=\mu_{0} \mathbf{H}$, el campo $\mathbf{D}=\varepsilon_{0} \mathbf{E}$, con $\varepsilon_{0}$ y $\mu_{0}$ la permeabilidad magnética y la permitividad eléctrica del vacío respectivamente, y $\varepsilon_{0} \mu_{0}=c^{-2}$. Los campos B y $\mathbf{E}$ pueden ser determinados a partir de un potencial vector $\mathbf{A}(\mathbf{r}, t)$ mediante las siguientes relaciones

$$
\begin{aligned}
& \mathbf{B}=\nabla \times \mathbf{A}, \\
& \mathbf{E}=-\frac{\partial \mathbf{A}}{\partial t} .
\end{aligned}
$$

Dado que las Ecuaciones de Maxwell (A.1) son invariantes de gauge se utilizará, por conveniencia, el gauge de Coulomb, esto es

$$
\nabla \cdot \mathbf{A}=0
$$


Si se reemplaza esta condición de gauge en las Ecuaciones (A.2) y (A.3) se obtiene la ecuación de onda que satisface el potencial vector $\mathbf{A}(\mathbf{r}, t)$

$$
\nabla^{2} \mathbf{A}(\mathbf{r}, t)=\frac{1}{c^{2}} \frac{\partial^{2} \mathbf{A}(\mathbf{r}, t)}{\partial t^{2}}
$$

El potencial vector se puede escribir como suma de dos términos complejos

$$
\mathbf{A}(\mathbf{r}, t)=\mathbf{A}^{(+)}(\mathbf{r}, t)+\mathbf{A}^{(-)}(\mathbf{r}, t)
$$

Donde $\mathbf{A}^{(+)}(\mathbf{r}, t)$ contiene todas las amplitudes que varían temporalmente como $e^{-i \omega t}($ con $\omega>0)$ y $\mathbf{A}^{(-)}(\mathbf{r}, t)$ contiene todas las amplitudes que varían como $e^{i \omega t}$, así se tiene $\mathbf{A}^{(-)}(\mathbf{r}, t)=\left(\mathbf{A}^{(+)}(\mathbf{r}, t)\right)^{*}$. Por conveniencia se utilizará un conjunto de variables discretas en lugar de un continuo. Si se restringe el campo a un cierto volumen y se expande el potencial vector en términos de un conjunto ortogonal de funciones se obtiene

$$
\mathbf{A}^{(+)}(\mathbf{r}, t)=\sum_{k} c_{k} \mathbf{u}_{k}(\mathbf{r}) e^{-i \omega_{k} t}
$$

Donde los coeficientes de Fourier $c_{k}$ son constantes para el campo libre. El conjunto de funciones del k-ésimo modo $\mathbf{u}_{k}(\mathbf{r})$ que corresponden a las frecuencias $\omega_{k}$ satisfacen la ecuación de onda

$$
\left(\nabla^{2}+\frac{\omega_{k}^{2}}{c^{2}}\right) \mathbf{u}_{k}(\mathbf{r})=0 .
$$

Este conjunto de funciones deben satisfacer la condición de transversalidad

$$
\nabla \mathbf{u}_{k}(\mathbf{r})=0
$$

Las funciones de los modos forman un conjunto ortonormal y completo

$$
\int_{V} \mathbf{u}_{k}^{*}(\mathbf{r}) \mathbf{u}_{k^{\prime}}(\mathbf{r})=\delta_{k, k^{\prime}}
$$

Las funciones de los modos dependen de las condiciones de borde del volumen físico que está bajo consideración. Por ejemplo, las ondas planas son funciones adecuadas para un volumen cúbico de longitud $L$, las cuales se escriben como

$$
\mathbf{u}_{k}(\mathbf{r})=L^{-3 / 2} \check{\mathbf{e}}_{\lambda} e^{i \mathbf{k} \cdot \mathbf{r}} .
$$


Donde $\check{\mathbf{e}}_{\lambda}(\lambda=1,2)$ son los vectores de polarización unitarios que satisfacen

$$
\check{\mathbf{e}}_{\lambda} \cdot \check{\mathbf{e}}_{\lambda^{\prime}}=\delta_{\lambda \lambda^{\prime}}, \quad \check{\mathbf{e}}_{\lambda} \cdot \mathbf{k}=0, \quad \check{\mathbf{e}}_{1} \times \check{\mathbf{e}}_{2}=\mathbf{k} /|\mathbf{k}|
$$

En el índice $k$ están contenidas las variables discretas: la polarización y las tres componentes cartesianas del vector de onda $\mathbf{k}$. Cada componente del vector de onda $\mathbf{k}$ toma valores

$$
k_{x}=\frac{2 \pi n_{x}}{L}, k_{y}=\frac{2 \pi n_{y}}{L}, \quad k_{z}=\frac{2 \pi n_{z}}{L}, \quad n_{x}, n_{y}, n_{z}=0, \pm 1, \pm 2, \ldots
$$

El vector polarización $\check{e}^{\lambda}$ debe ser perpendicular a $\mathbf{k}$ para satisfacer la condición de transversalidad (Ecuación (A.9)). El potencial vector puede ahora escribirse como

$$
\mathbf{A}(\mathbf{r}, t)=\sum_{k}\left(\frac{\hbar}{2 \omega_{k} \varepsilon_{0}}\right)^{1 / 2}\left[a_{k} \mathbf{u}_{k}(\mathbf{r}) e^{-i \omega_{k} t}+a_{k}^{\dagger} \mathbf{u}_{k}^{*}(\mathbf{r}) e^{i \omega_{k} t}\right] .
$$

Utilizando la relación (A.3) se obtiene la expresión para el campo electromagnético asociado

$$
\mathbf{E}(\mathbf{r}, t)=i \sum_{k}\left(\frac{\hbar \omega_{k}}{2 \varepsilon_{0}}\right)^{1 / 2}\left[a_{k} \mathbf{u}_{k}(\mathbf{r}) e^{-i \omega_{k} t}-a_{k}^{\dagger} \mathbf{u}_{k}^{*}(\mathbf{r}) e^{i \omega_{k} t}\right]
$$

Los factores de normalización se eligieron de forma que las amplitudes $a_{k}$ y $a_{k}^{\dagger}$ sean adimensionales.

En la teoría clásica del electromagnetismo estas amplitudes son números complejos. La cuantización del campo electromagnético se realiza al tomar los factores $a_{k} \mathrm{y} a_{k}^{\dagger}$ como operadores adjuntos. Debido a que los fotones son bosones las relaciones de conmutación apropiadas para los operadores $a_{k} \mathrm{y} a_{k}^{\dagger}$ son

$$
\left[a_{k}, a_{k^{\prime}}\right]=\left[a_{k}^{\dagger}, a_{k^{\prime}}^{\dagger}\right]=0, \quad\left[a_{k}, a_{k^{\prime}}^{\dagger}\right]=\delta_{k, k^{\prime}}
$$

El comportamiento dinámico de las amplitudes del campo eléctrico pueden entonces ser descriptas por un conjunto independiente de osciladores armónicos que obedecen las relaciones de conmutación bosónicas. Los estados cuánticos de cada modo pueden ahora discutirse independientemente unos de otros. Y pueden ser descriptos por un vector de estado $\left|\Psi_{k}\right\rangle$ de un espacio de Hilbert apropiado para ese modo. El estado entero del campo puede ser definido en el espacio producto tensorial de todos los 
modos del espacio de Hilbert.

El Hamiltoniano del campo electromagnético libre esta dado por

$$
H=\frac{1}{2} \int\left(\varepsilon_{0} \mathbf{E}^{2}+\mu_{0} \mathbf{B}^{2}\right) .
$$

Si en esta expresión reemplazamos la Ecuación (A.15), para el campo electromagnético $\mathbf{E}(\mathbf{r}, t)$, y en forma análoga se introduce la expresión para $\mathbf{H}(\mathbf{r}, t)$, que se consigue de reemplazar en la Ecuación (A.2) la Ecuación (A.14), se obtiene la forma reducida para el Hamiltoniano

$$
H=\sum_{k} \hbar \omega_{k}\left(a_{k}^{\dagger} a_{k}+\frac{1}{2}\right)
$$

Esta expresión muestra que el Hamiltoniano del campo electromagnético es igual a la suma del número de fotones en cada modo multiplicado por la energía de un fotón de modo, más $(1 / 2) \hbar \omega_{k}$ que representa la energía de las fluctuaciones del vacío en cada modo. 


\section{Apéndice B}

\section{Estados del campo electromagnético}

\section{B.1. Estados número}

El Hamiltoniano de la Ecuación (A.17) tiene autovalores $\hbar \omega_{k}\left(n_{k}+\frac{1}{2}\right)$ donde $n_{k}$ es un número natural. Los autoestados se pueden escribir como $\left|n_{k}\right\rangle$ y son conocidos como estados de Fock o estados número. Ellos son autoestados del operador número $N_{k}=a_{k}^{\dagger} a_{k}$, esto es

$$
a_{k}^{\dagger} a_{k}\left|n_{k}\right\rangle=n_{k}\left|n_{k}\right\rangle
$$

El estado fundamental del oscilador (o el estado de vacío del campo) es definido por

$$
a_{k}|0\rangle=0
$$

De las Ecuaciones (A.17) y (B.1) se puede ver que la energía del estado de vacío es

$$
\langle 0|H| 0\rangle=\frac{1}{2} \sum_{k} \hbar \omega_{k}
$$

Debido a que no están acotadas superiormente las frecuencias, en la suma sobre los distintos modos de los campos, la energía del estado fundamental es infinita. Esto presenta una dificultad conceptual. Sin embargo, debido a que en los experimentos se mide el cambio total de energía del campo electromagnético, la energía infinita de punto cero no conduce a ninguna divergencia en la práctica. Una discusión más profunda sobre este punto se puede ver en el libro de Power (1964). 
$a_{k}^{\dagger}$ y $a_{j}$ son los operadores de creación y destrucción del oscilador armónico. En términos de fotones, representan los operadores de creación o aniquilación de fotones con vector de onda $\mathbf{k}$ y polarización $\check{e}^{\lambda}$. Así, la aplicación de de los operadores de aniquilación y creación sobre un estado número da como resultado

$$
a_{k}\left|n_{k}\right\rangle=n_{k}^{1 / 2}\left|n_{k}-1\right\rangle, \quad a_{k}^{\dagger}\left|n_{k}\right\rangle=\left(n_{k}+1\right)^{1 / 2}\left|n_{k}+1\right\rangle .
$$

Estos estos estados excitados pueden ser obtenidos del vacío por aplicación sucesiva de operadores de creación

$$
\left|n_{k}\right\rangle=\frac{\left(a_{k}^{\dagger}\right)^{n_{k}}}{\left(n_{k} !\right)^{1 / 2}}|0\rangle, \quad n_{k}=0,1,2 \ldots
$$

Los estados número así obtenidos son ortonormales

$$
\left\langle n_{k^{\prime}} \mid m_{k}\right\rangle=\delta_{k, k^{\prime}} \delta_{n, m}
$$

y completos

$$
\sum_{n_{k}}\left|n_{k}\right\rangle\left\langle n_{k}\right|=I
$$

Debido a que la norma de estos vectores es finita, ellos forman un conjunto completo de vectores (de una base) del espacio de Hilbert.

\section{B.2. Estados coherentes}

Una base mas apropiada para ser utilizada en el campo de la óptica cuántica es la de estados coherentes del campo electromagnético (Glauber, 1963). Los estados coherentes tienen un número indefinido de fotones, esto permite tener en forma más precisa la fase, a diferencia de lo que ocurre con los estados número donde la fase es completamente aleatoria. Para estos estados, el producto de incertezas en amplitud y fases es el mínimo permitido por el principio de incerteza. En este sentido se puede decir que los estados coherentes son los estados cuánticos del campo electromagnético que más cerca se encuentran de una descripción clásica del campo. En el laboratorio, los estados coherentes se pueden generar por un láser altamente 
estabilizado que funcione por encima del umbral.

A continuación se muestra un breve resumen de los estados coherente. En forma análoga a la vista en la Sección 1.2, estos estados son fácilmente generados utilizando el operador unitario de desplazamiento.

$$
D(\alpha)=e^{\left(\alpha a^{\dagger}-\alpha^{*} a\right)}
$$

donde $\alpha$ es un número complejo. Utilizando la relación de Baker-Campbell-Hausdorff

$$
e^{A+B}=e^{A} e^{B} e^{-[A, B] / 2},
$$

donde se utilizó la condición $[A,[A, B]]=[B,[A, B]]=0$, se puede escribir $D(\alpha)$ como

$$
D(\alpha)=e^{-|\alpha|^{2} / 2} e^{\alpha a^{\dagger}} e^{-\alpha^{*} a} .
$$

El operador de desplazamiento $D(\alpha)$ posee las siguientes propiedades

$$
\begin{array}{r}
D^{\dagger}(\alpha)=D^{-1}(\alpha)=D(-\alpha), \\
D^{\dagger}(\alpha) a D(\alpha)=a+\alpha, \\
D^{\dagger}(\alpha) a^{\dagger} D(\alpha)=a^{\dagger}+\alpha^{*} .
\end{array}
$$

El estado coherente $|\alpha\rangle$ se genera aplicando el operador $D(\alpha)$ sobre el estado de vacío

$$
|\alpha\rangle=D(\alpha)|0\rangle
$$

Los estados coherentes son autoestados del operador $a$. Esto se deduce de la siguiente forma:

$$
D^{\dagger}(\alpha) a|\alpha\rangle=D^{\dagger}(\alpha) a D(\alpha)|0\rangle=(a+\alpha)|0\rangle=\alpha|0\rangle
$$

Multiplicando ambos lados por $D(\alpha)$ se obtienen la ecuación de autovalores

$$
a|\alpha\rangle=\alpha|\alpha\rangle
$$

Debido a que el operador $a$ es no-hermítico sus autovalores $\alpha$ son complejos.

Otra propiedad útil se deriva utilizando la relación que se encuentra en la Ecuación 


$$
D(\alpha+\beta)=D(\alpha) D(\beta) e^{-i \operatorname{Im}\left\{\alpha \beta^{*}\right\}}
$$

Los estados coherentes contienen un número indefinido de fotones. Esto puede apreciarse, si se considera la expansión en estado número de los estados coherentes

$$
|\alpha\rangle=e^{-|\alpha|^{2} / 2} \sum_{n} \frac{\alpha^{n}}{(n !)^{1 / 2}}|0\rangle .
$$

A partir de esta expresión es fácil mostrar que la distribución de probabilidades de fotones en un estado coherente corresponde a una distribución de Poisson

$$
P(n)=|\langle n \mid \alpha\rangle|^{2}=\frac{|\alpha|^{2 n} e^{-|\alpha|^{2}}}{n !},
$$

donde $|\alpha|^{2}$ es el número medio de fotones $\bar{n}=\left\langle\alpha\left|a^{\dagger} a\right| \alpha\right\rangle=|\alpha|^{2}$.

Dos estados coherentes distintos $|\alpha\rangle,|\beta\rangle$ no son ortogonales entre sí, ya que

$$
\langle\alpha \mid \beta\rangle=e^{\left[-\frac{1}{2}\left(|\alpha|^{2}+|\beta|^{2}\right)+\alpha \beta^{*}\right]},
$$

del cual se puede obtener el producto escalar entre ellos

$$
|\langle\alpha \mid \beta\rangle|^{2}=e^{-|\alpha-\beta|^{2}}
$$

De aquí se puede ver que los estados coherentes $|\alpha\rangle,|\beta\rangle$ se vuelven aproximadamente ortogonales en el límite $|\alpha-\beta| \gg 1$. Los estados coherentes forman una base continua de estados, que de hecho, es sobre completa. Se puede comprobar que se satisface la relación

$$
\frac{1}{\pi} \int|\alpha\rangle\langle\alpha| d \alpha=1
$$

\section{B.3. Estados comprimidos}

Una clase general de estados del campo electromagnético son los llamados estados comprimidos. En general, un estado comprimido puede tener un ruido menor en una de sus cuadraturas que un estado coherente. Para satisfacer el requerimiento de que el producto de incertezas sea mínimo la incerteza de la otra cuadratura deberá ser 
mayor que la que se tenía para un estado coherente.

A continuación se considera las propiedades de un modo simple del campo. Primero se reescribe el operador de aniquilación del campo como una combinación lineal de dos operadores Hermíticos

$$
a=\frac{X_{1}+i X_{2}}{2}, \quad a^{\dagger}=\frac{X_{1}-i X_{2}}{2} .
$$

$X_{1}$ y $X_{2}$, que son las partes real e imaginaria de la amplitud compleja. Estos operadores obedecen la relación de conmutación

$$
\left[X_{1}, X_{2}\right]=2 i
$$

Por el principio de incerteza se tiene la siguiente desigualdad

$$
\Delta X_{1} \Delta X_{2} \geq 1
$$

donde se utiliza $(\Delta A)^{2}=\left\langle A^{2}\right\rangle-\langle A\rangle^{2}$.

Los estados de minima incerteza, esto es que se sature la igualdad de la relación (B.25), y que además cumplan

$$
\Delta X_{1}=\Delta X_{2}=1
$$

definen una familia de estados llamados estados coherentes. Un estado coherente $|\alpha\rangle$ tiene como valor medio una amplitud compleja $\alpha$ y es un estado de incerteza mínimo para $X_{1}$ y $X_{2}$, con incertezas iguales en ambas fases de las cuadraturas. Estas incertezas pueden ser representadas por un "circulo de error" en un plano de amplitudes complejas cuyos ejes son $X_{1}$ y $X_{2}$. El centro del circulo de error yace en el punto $\frac{1}{2}\left\langle X_{1}+i X_{2}\right\rangle=\alpha$ y tiene por radio $\Delta X_{1}=\Delta X_{2}=1$ que da cuenta de las incertezas en $X_{1}$ y $X_{2}$ (Figura B.1 (a)). Se hace evidente que existe una familia de estados de mínima incerteza definidos por la relación $\Delta X_{1} \Delta X_{2}=1$. Si se grafica $\Delta X_{1}$ contra $\Delta X_{2}$, los estados de mínima incerteza yacen sobre una hipérbola (Figura 1.5). Solo los estados a la derecha de la hipérbola son estados físicos, ya que los que se encuentran a la izquierda no satisfacen el principio de incerteza. El punto $(1,1)$ representa a los estados coherentes y los estados corresponden a la región sombreada 

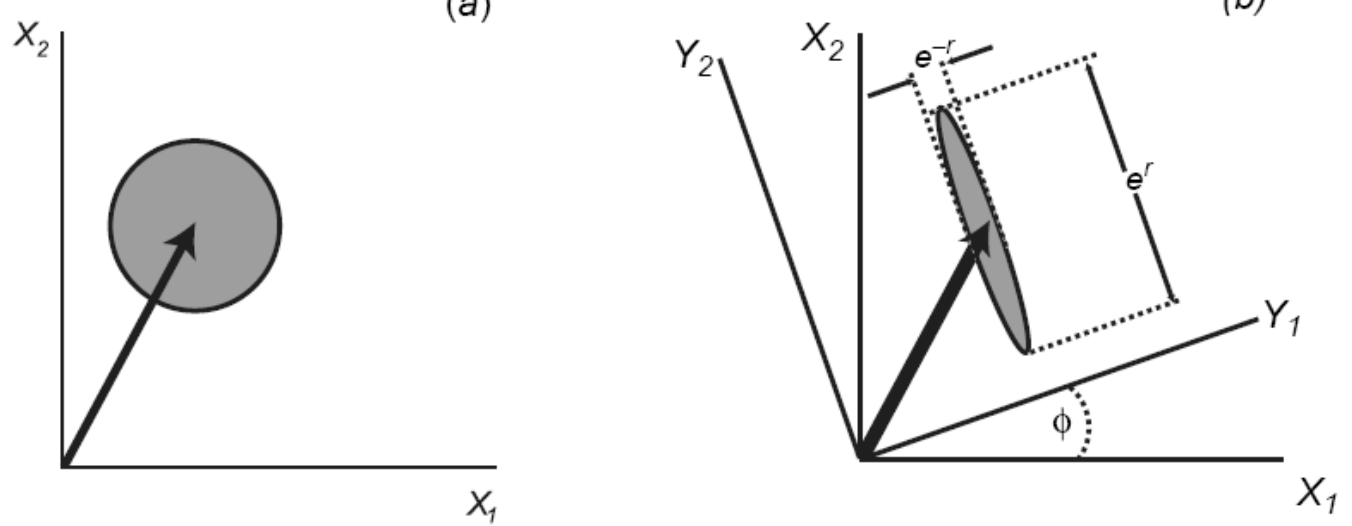

Figura B.1: Representación en el espacio de fases mostrando los contornos de incertezas para (a) Estado coherente $|\alpha\rangle$ y (b) Estado comprimido $|\alpha, \varepsilon\rangle$.

en la Figura 1.5, presentan una reducción de incerteza por debajo del límite cuántico estándar (en este caso el valor del conmutador es igual a uno). Dichos estados son los llamados estados comprimidos (Walls, 1986). Ellos pueden ser generados utilizando el operador unitario de compresión (Caves, 1981)

$$
S(\varepsilon)=e^{\frac{1}{2}\left(\varepsilon^{*} a^{2}-\varepsilon a^{\dagger 2}\right)},
$$

$\operatorname{con} \varepsilon=r e^{2 i \phi}$.

El operador de compresión cumple

$$
S^{\dagger}(\varepsilon)=S^{-1}(\varepsilon)=S(-\varepsilon)
$$

y tiene las siguientes propiedades de transformación:

$$
\begin{gathered}
S^{\dagger}(\varepsilon) a S(\varepsilon)=a \cosh r-a^{\dagger} e^{-2 i \phi} \sinh r \\
S^{\dagger}(\varepsilon) a^{\dagger} S(\varepsilon)=a^{\dagger} \cosh r-a e^{-2 i \phi} \sinh r \\
S^{\dagger}(\varepsilon)\left(Y_{1}+i Y_{2}\right) S(\varepsilon)=Y_{1} e^{-r}+i Y_{2} e^{r}
\end{gathered}
$$

Donde $Y_{1}+i Y_{2}=\left(X_{1}+i X_{2}\right) e^{-i \phi}$ es la amplitud compleja rotada. El operador de compresión atenúa una componente de la amplitud compleja rotada, y amplifica la otra. El grado de amplificación y atenuación está dado por $r=|\varepsilon|$, al cual se le llama factor de squeezing. El estado comprimido $|\alpha, \varepsilon\rangle$ se obtiene primero comprimiendo 
el vacío y luego desplazándolo

$$
|\alpha, \varepsilon\rangle=D(\alpha) S^{\dagger}(\varepsilon)|0\rangle
$$

Los estados comprimidos tienen los siguientes valores medios y varianzas

$$
\begin{aligned}
\left\langle X_{1}+i X_{2}\right\rangle & =\left\langle Y_{1}+i Y_{2}\right\rangle e^{i \phi} 2 \alpha \\
\Delta Y_{1} & =e^{-r} \\
\Delta Y_{2} & =e^{r}, \\
\langle N\rangle=\left|\alpha^{2}\right|+\sinh ^{2} r,(\Delta N)^{2} & =\left|\alpha \cosh r-\alpha^{*} e^{2 i \phi} \sinh r\right|^{2}+2 \cosh ^{2} r \sinh ^{2} r .
\end{aligned}
$$

Así los estados comprimidos tienen incertezas distintas para $Y_{1}$ e $Y_{2}$ como se ve en la elipse de error en la Figura 1.5. 


\section{Apéndice $\mathrm{C}$}

\section{Átomos fríos}

\section{C.1. Principios básicos del enfriamiento Doppler}

Supongamos un átomo libre moviéndose en la en la dirección del eje- $x$ con velocidad $v_{x}$, como se muestra en la Figura C.1. Si el átomo interactúa con un haz láser que se propaga con sentido contrario y que posee una frecuencia $\nu_{L} \equiv c / \lambda$, sintonizada con la de resonancia del átomo. Entonces se puede escribir

$$
\nu_{L}=\nu_{0}+\delta
$$

donde $\nu_{0}$ es la frecuencia de transición atómica en reposo y $\delta \ll \nu_{0}$. En el sistema de referencia del átomo, la fuente se mueve hacia el átomo y la frecuencia es corrida hacia una más alta por efecto Doppler. El corrimiento en frecuencia Doppler esta dado por

$$
\nu_{L}^{\prime}=\nu_{L}\left(1+\frac{v_{x}}{c}\right)=\left(\nu_{0}+\delta\right)\left(1+\frac{v_{x}}{c}\right) \approx \nu_{0}+\delta+\frac{v_{x}}{c} \nu_{0},
$$

donde se utilizó que $v_{x} \ll c$. Es evidente que si se elige

$$
\delta=-\nu_{0} \frac{v_{x}}{c}=-\frac{v_{x}}{\lambda}
$$

se encuentra que $\nu_{L}^{\prime}=\nu_{0}$. Cuando se satisface esta condición, el láser entra en resonancia con el átomo que se mueve en la dirección $x$.

Cuando el átomo absorbe un fotón, del haz láser, pasa a estar en un estado excitado y experimenta un cambio en la cantidad de movimiento

$$
\delta p_{x}=-\frac{h}{\lambda}
$$




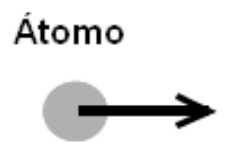

$v_{\mathrm{x}}$
Haz Laser

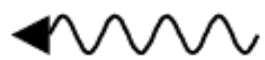

$\nu_{\mathrm{L}}$

Figura C.1: En el enfriamiento Doppler, la frecuencia del láser es sintonizada por debajo de la resonancia atómica una cantidad $\delta$. La frecuencia vista por el átomo en movimiento hacia el láser es incrementada por el efecto Doppler en $\nu_{0}\left(v_{x} / c\right)$.

El átomo en el estado excitado tiene una vida media $\tau$. Si no hay emisión estimulada, el átomo se desexcitará emitiendo un fotón de igual frecuencia en dirección aleatoria. Si el proceso se repite un número grande de veces, el cambio neto de la cantidad de movimiento por desexcitación promedia a cero. Por lo cual, el valor medio de la variación del momento por proceso es el de la Ecuación C.4. Así, la fuerza de roce que experimenta un átomo en estas condiciones es

$$
F_{x}=\frac{d p_{x}}{d t} \approx \frac{\Delta p_{x}}{2 \tau}=-\frac{h}{2 \lambda \tau} .
$$

Donde el factor dos en el denominador proviene del hecho de que a altas intensidades la población del estado excitado y el fundamental valen aproximadamente $N_{0} / 2$, donde $N_{0}$ es el número total de átomos

Para obtener una estimación, de la menor temperatura que se puede obtener mediante este tipo de método de enfriamiento, se puede pensar que el enfriamiento Doppler funcionara hasta que el ancho natural de la línea sea comparable con la desintonización en frecuencia del láser. En estas condiciones la energía térmica del átomo será $k_{b} T_{\min } \sim h \Delta \nu$, utilizando la relación entre al ancho de línea y el tiempo de vida obtenemos

$$
T_{\min } \sim \frac{h}{k_{b} \tau} .
$$




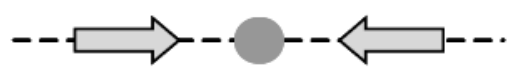

Figura C.2: Dos haces láser que se propagan en direcciones opuestas producen el efecto de melaza óptica.

\section{C.2. Melaza óptica (Optical molasses)}

Los resultados de la sección anterior pueden ser considerados para estimar el orden de magnitud de la temperatura mínima que se puede alcanzar con esta técnica. En esta sección se muestra con un poco más de detalle el proceso de enfriamiento y se dará una estimación de cuál es el límite mínimo de temperatura que se puede obtener.

Si se considera un haz láser con intensidad óptica $I$ y desintonización $\Delta \equiv 2 \pi \delta$ en unidades de frecuencia angular, interactuando con un átomo con velocidad $v_{x}$ con respecto a la fuente del láser. Como en la Ecuación (C.5), la fuerza de roce $F_{x}$ está dado por el cambio de momento en un ciclo de absorción-emisión multiplicado por la proporción neta con la que ocurre dicho cambio en un ciclo:

$$
F_{x}=-\hbar k \times R(I, \Delta)
$$

donde $k \equiv 2 \pi / \lambda$ es el vector de onda del fotón. $R(I, \lambda)$ se la denomina porción neta de absorción y es igual a la proporción de absorción menos la proporción de emisión estimulada. Está dada por:

$$
R(I, \Delta)=\frac{\gamma}{2}\left(\frac{I / I_{s}}{1+I / I_{s}+\left[2\left(\Delta+k v_{x}\right) / \gamma\right]}\right),
$$

donde $\gamma \equiv 1 / \tau$ es el ensanchamiento natural en unidades de momento angular, y $I_{s}$ es la intensidad de saturación de la transición. En el límite de altas intensidades esta expresión converge a $\gamma / 2$, lo que justifica el factor dos en el denominador de la expresión (C.5). En la sección anterior vimos que el límite de enfriamiento de átomos con un solo láser se da cuando la desintonización es del orden del ensanchamiento de línea. En estas condiciones los átomos que se mueven en la dirección $-x$ experimen- 


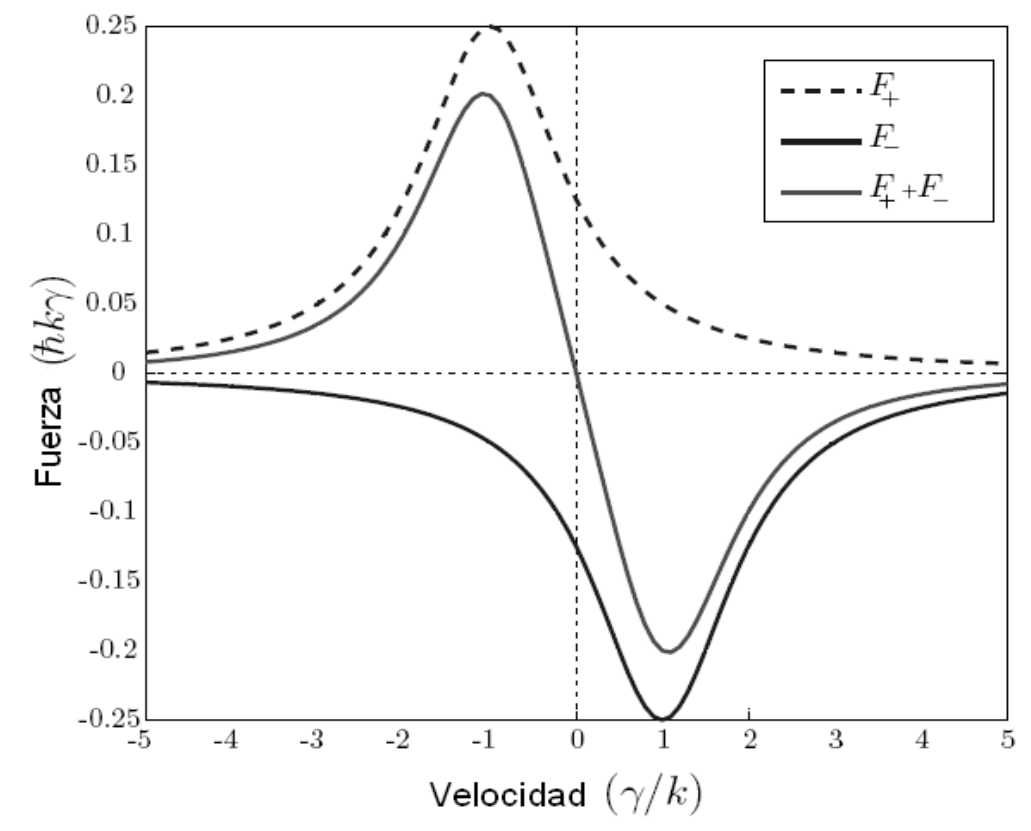

Figura C.3: Fuerza que experimenta un átomo cuando es sometido a dos haces láser que se propagan en direcciones opuestas.

tan aceleración y a través de colisiones recalientan a los que se mueven en dirección $+x$. Para conseguir temperaturas menores se pueden utilizar dos hazes láser como el de la Figura C.2. En este esquema los átomos experimentan distintas fuerzas de cada láser y la fuerza neta viene dada por la expresión

$$
F_{x}=F_{+}+F_{-},
$$

donde $F_{ \pm}$se refiere a la fuerza provocada por el haz láser que se propaga en la dirección $\pm x$, respectivamente. Esta configuración es apta para enfriar átomos que se mueven en ambas direcciones. Para átomos que se mueven en dirección $+x$ se tiene $F_{-} \gg F_{+}$y vice versa para los que se mueven con dirección $-x$. En el límite de bajas temperaturas, cuando $k v_{x} \ll \Delta$, y $\left|k v_{x}\right| \ll \gamma$, la fuerza resultante está dada 
por la expresión

$$
F_{x}(I, \Delta)=\frac{8 \hbar k^{2} \Delta}{\gamma}\left(\frac{I / I_{s}}{\left[1+I / I_{s}+(2 \Delta / \gamma)^{2}\right]^{2}}\right) v_{x} .
$$

Independientemente de la dirección de $v_{x}$, la fuerza es de la forma:

$$
F_{x}=-\alpha v_{x}
$$

donde $\alpha$ es la contante de amortiguamiento, que esta dada por

$$
\alpha=-\frac{8 \hbar k^{2} \Delta}{\gamma}\left(\frac{I / I_{s}}{\left[1+I / I_{s}+(2 \Delta / \gamma)^{2}\right]^{2}}\right) .
$$

Cuando $\Delta$ es negativo, $\alpha$ es positivo y por lo tanto el movimiento de los átomos esta amortiguado en ambas direcciones. Por esta razón es que a este arreglo experimental se lo llamó "melasa óptica".

Por más que la fuerza viscosa reduzca la velocidad media a cero, el átomo permanece realizando un paseo aleatorio con velocidad media cuadrática distinta de cero. Esto se debe a que el átomo con velocidad media igual a cero absorbe con igual probabilidad fotones de cualquiera de los dos haces. Para calcular el límite con el cual se puede utilizar este método para enfriar se debe igualar la pérdida de energía en función del tiempo por el enfriamiento con el calor que ingresa al sistema. A partir de esta condición se puede mostrar que la temperatura mínima en el límite de intensidades bajas esta dado por

$$
T_{\min }=\frac{\hbar \gamma}{2 k_{b}}=\frac{\hbar}{2 k_{b} \tau}
$$

cuando $\Delta=-\gamma / 2$. Esta es llamada temperatura Doppler límite, que normalmente es del orden de los $\mu K$.

\section{C.3. Enfriamiento sub-Doppler}

El límite de temperatura dado por la Ecuación (C.13) no es el mínimo que se puede obtener mediante las técnicas de enfriamiento láser. Experimentos cuidadosos realizados en la década del 80 mostraron que la temperatura que se podía obtener era aun menor. Esta discrepancia condujo a un nuevo mecanismo de enfriamiento 
llamado Sisyphus. Una explicación simplificada de este mecanismo se muestra en la Figura C.4. Si se considera un átomo alcalino con su estado fundamental ${ }^{2} S_{1 / 2}$ moviéndose en la dirección $+x$ y realizando transiciones al estado excitado ${ }^{2} P_{3 / 2}$ bajo la influencia de dos haces láser que se propagan en direcciones contrarias Figura C.2. Las franjas de interferencia de los láseres formaran modulaciones periódicas de las energías del estado fundamental debido al efecto Stark. La luz induce corrimientos de los subniveles magnéticos $M_{j}= \pm 1 / 2$ que difieren en una fase de $\pi$ radianes, como muestra la Figura C.4. Si el átomo permanece en el mismo subnivel, este intercambiara energía cinética por potencial (y viceversa), a lo largo de su trayecto sin pérdida de energía total (por ejemplo $1 \rightarrow 2 \rightarrow 5$ ). Si los lasers se sintonizan de forma que el átomo solo absorba energía cuando se encuentra en un máximo de potencial (puntos 2 y 4) puede ocurrir los siguiente: a) al decaer vuelve al estado inicial, por lo cual no hay perdida de energía por parte del átomo, b) decae al estado de menor energía (punto 3 o 5) por lo cual habrá perdida de energía cinética por parte del átomo. En el caso de un átomo siguiendo el camino $1 \rightarrow 2 \rightarrow 3 \rightarrow 4 \rightarrow 5 \rightarrow \ldots$, la diferencia en energía de los fotones absorbidos y emitidos es tomada de la energía cinética del átomo, conduciendo a un efecto de enfriamiento.

La energía mínima que se puede alcanzar con el enfriamiento Sisyphus esta dado por el límite de retroceso. Los átomos están constantemente emitiendo fotones de longitud de onda $\lambda$ en direcciones aleatorias. Por lo cual el átomo retrocede una cantidad de momento $h / \lambda$ y obtiene una energía térmica proveniente de un proceso aleatorio que vale

$$
T_{r e t}=\frac{h^{2}}{m k_{b} \lambda^{2}}
$$

El orden de esta temperatura de retroceso en experimentos, como puede ser los realizados con átomos de cesio, son del $\mu K$. 


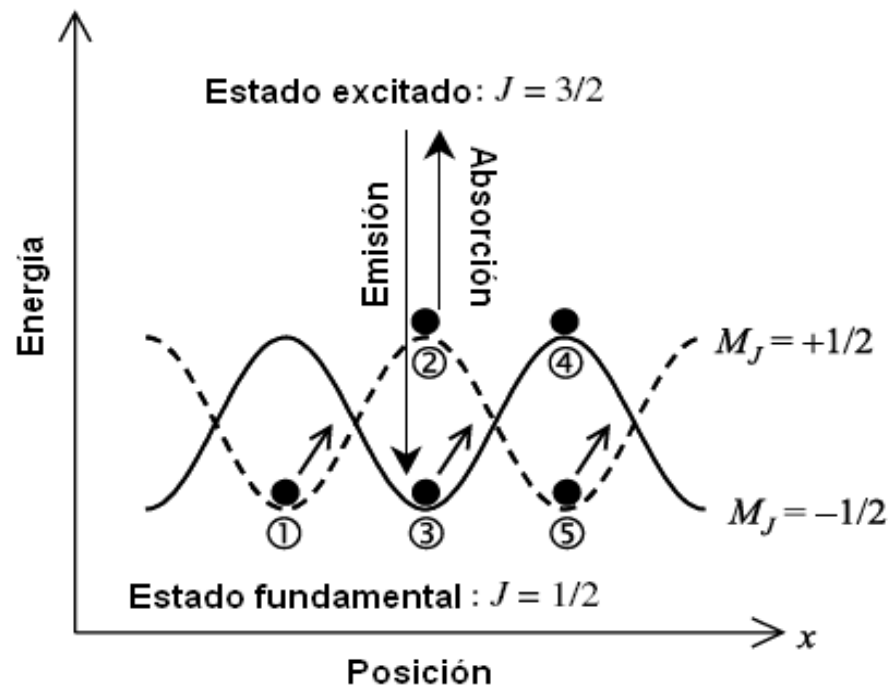

Figura C.4: Enfriamiento Sisyphus para una transición $J=1 / 2 \rightarrow 3 / 2$ en un átomo alcalino. El átomo se mueve en la dirección $x$, e interactúa con dos haces láser que se propagan en direcciones contrarias como en la Figura C.2. La energía de los subniveles $M_{j}= \pm 1 / 2$ del estado fundamental $J=1 / 2$ varía sinusoidalmente con la posición en el patrón de interferencia generado por los lasers. El láser es sintonizado en frecuencia de modo que el átomo solo puede realizar una transición al estado excitado cuando se encuentra en la cima de un máximo de potencial (posición 2 y 4). El átomo en el estado excitado puede reemitir al mismo subnivel, o a uno menor (posición 3 y 5). En el caso de un átomo siguiendo el camino $1 \rightarrow 2 \rightarrow 3 \rightarrow 4 \rightarrow 5 \rightarrow$.... la diferencia en energía de los fotones absorbidos y emitidos es tomada de la energía total del átomo, conduciendo a un efecto de enfriamiento. 


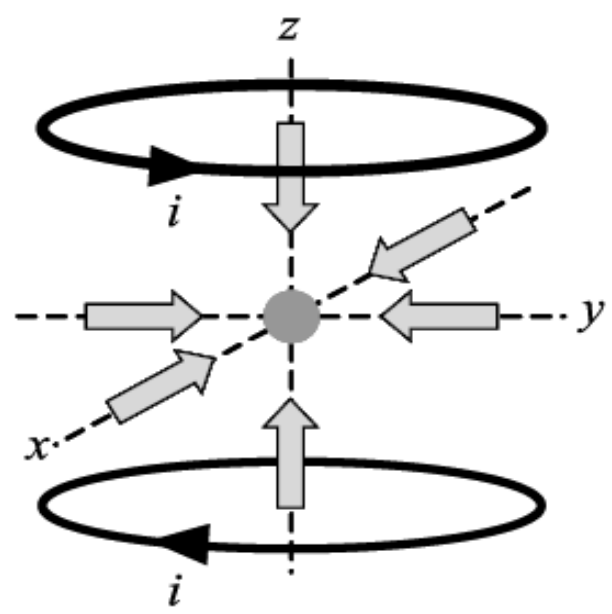

Figura C.5: Esquema de una trampa magneto-óptica. Dos haces opuestos se encuentran en las direcciones $x, y, z$, para anular las componentes de la velocidad de los átomos. Los campos magnéticos son generados por dos bobinas que llevan dos corrientes iguales con direcciones opuesta, atrapando a los átomos con $M_{j}>0$ en el minimo del potencial generado donde se hacen coincidir con la intersección de los haces láser.

\section{C.4. Trampas magneto-ópticas}

El arreglo de la Figura C.2 reduce la componente de la velocidad de los átomos en la dirección en la que se propagan los lasers, pero no tiene efecto en las restantes. Para reducir las tres componentes de la velocidad de los átomos y acotarlos en un pequeño volumen se utiliza una trampa Magneto-óptica como la que se muestra en la Figura C.5. Estas trampas poseen tres dispositivos como los de la FiguraC.2, para generar el efecto de melaza óptica en las tres coordenadas, y un campo magnético cuadrupolar generado por dos bovinas (Figura C.5) para generar un mínimo de potencial en el centro. El campo magnético crea un potencial atractivo para los los átomos, cuyo estado sea $M_{j}>0$. 


\section{Agradecimientos}

Quiero agradecer a todas las personas y entidades que de una forma u otra han hecho posible esta tesis:

- a la Universidad Nacional de La Plata, al IFLP, y al CONICET por proveer los recursos que hicieron posible el desarrollo de esta tesis;

- a Osvaldo y Marta por la motivación y el entusiasmo que me han transmitido, la infinita paciencia, y la predisposición para trabajar;

- a mis compañeros de la facultad y amigos por compartir las experiencias diarias;

- a mi familia: Mis padres Manuel y Angela, y mis hermanas Alicia y Noelia, por apoyarme en todo desde que tengo memoria;

- y a Marina que me acompañó en el proceso de elaboración de este trabajo. 


\section{Bibliografía}

Affronte, M., Ghirri, A., Carretta, S., Amoretti, G., Piligkos, S., Timco, G., and Winpenny, R.: 2004, Appl. Phys. Lett. 84, 3468

Agarwal, G. and Patnaik, A.: 2001, Phys. Rev. A 63, 438051

Ahn, J., Weinacht, T., and Bucksbaum, P.: 2000, Science 287, 463

Barberis-Blostein, P. and Bienert, M.: 2009, Phys. Rev. A 79, 063824

Barnett, S. and Knight, P.: 1986, Phys. Rev. A 33, 2444

Brattke, S., Kallmann, U., and Hartmann, W.: 1998, Eur. J. Phys. D 3, 159

Brennen, G., Caves, C., Jessen, P., and Deutsch, I.: 1999, Phys. Rev. Lett. 82, 1060

Brey, L., Fertig, H., and Das Sarma, S.: 2007, Phys. Rev. Lett. 99, 116802

Briegel, H., Calarco, T., Jaksch, D., Cirac, J., and Zoller, P.: 2000, J. Mod. Optics 47, 415

Brion, E., Mølmer, K., and Saffman, M.: 2007, Phys. Rev. Lett. 99, 260501

Buley, E. and Cummings, F.: 1964, Phys. Rev. 134, A1454

Campos Venuti, L., Degli Esposti Boschi, C., and Roncaglia, M.: 2007, Phys. Rev. Lett. 99, 060401

Cardimona, D., Alsing, P., Mozer, H., and Rhodes, C.: 2009, Phys. Rev. A 79, 063817

Carretta, S., Van Slageren, J., Guidi, T., Liviotti, E., Mondelli, C., Rovai, D., Cornia, A., Dearden, A., Carsughi, F., Affronte, M., Frost, C., Winpenny, R., Gatteschi, D., Amoretti, G., and Caciuffo, R.: 2003, Phys. Rev. B 67, 094405

Caves, R.: 1981, Phys. Rev. D 23, 1693

Cerf, N., Leuchs, G., and Polzik, E.: 2007, Quantum Information with Continuous 
Variables of Atoms and Light, Imperial College Press

Cirac, J. and Zoller, P.: 1995, Phys. Rev. Lett. 74, 4091

Civitarese, O. and Reboiro, M.: 1998, Phys. Rev. C 57, 3055

Civitarese, O. and Reboiro, M.: 1999, Phys. Rev. C 60, 343021

Civitarese, O. and Reboiro, M.: 2006, Phys. Lett. A 357, 224

Civitarese, O. and Reboiro, M.: 2010, Phys. Lett. A 374, 4664

Civitarese, O., Reboiro, M., Rebon, L., and Tielas., D.: 2008, Rev. Mex. Fis. 54, 24

Civitarese, O., Reboiro, M., Rebon, L., and Tielas, D.: 2009, Phys. Lett. A 373, 754

Civitarese, O., Reboiro, M., Rebon, L., and Tielas, D.: 2010a, Phys. Lett. A 374, 2117

Civitarese, O., Reboiro, M., Rebon, L., and Tielas., D.: 2010b, Phys. Lett. A 374, 424

Cohen-Tannoudji, C., Dupont-Roc, J., and Grynberg, G.: 1992, Atom-Photon Interactions, Wiley

Cummings, F.: 1965, Phys. Rev. 140, A1051

Dalton, B., Ficek, Z., and Knight, P.: 1994, Phys. Rev. A 50, 2646

Dantan, A., Pinard, M., Josse, V., Nayak, N., and Berman, P.: 2003, Phys. Rev. A 67, 458011

Deb, R., Abdalla, M., Hassan, S., and Nayak, N.: 2006, Phys. Rev. A 73, 053817

Dehmelt, H.: 1967, Adv. At. Mol. Phys. 3, 53

Deng, X., Porras, D., and Cirac, J. I.: 2005, Phys. Rev. A 72, 063407

Di Vincenzo, D.: 2000, Fortschr. Phys. 48, 771

Dicke, R.: 1954, Phys. Rev. 93, 99

Dittrich, J. and Inozemtsev, I.: 1997, J. Phys. A 30, L623

Drummond, P. and Ficek, Z.: 2004, Quantum Squeezing, Springer-Verlag

Duan, L., Demler, E., and Lukin, M.: 2003, Phys. Rev. Lett. 91, 904021

Echanis, S. R. and et al.: 2005, J. Opt. B 7, 548

Fermani, R., Scheel, S., and Knight, P.: 2007, Phys. Rev. A 75, 062905

Feynman, R.: 1957, J. Appl. Phys. 28(49)

Feynman, R.: 1982, Int. J. Theor. Phys. 21, 467 
Ficek, Z., Dalton, B., and Knight, P.: 1995, Phys. Rev. A 51, 4062

Ficek, Z. and Drummond, P.: 1991a, Phys. Rev. A 43, 6247

Ficek, Z. and Drummond, P.: 1991b, Phys. Rev. A 43, 6258

Frahm, H. and Inozemtsev, V.: 1994, J. Phys. A 27, L801

Furusawa, A. and Takei, N.: 2007, Phys. Rep. 443, 97

Garcia-Ripoll, J. and Cirac, J.: 2003, New J. Phys. 5, 76

Genes, C., Berman, P., and Rojo, A.: 2003, Phys. Rev. A 68, 043809

Gilmore, R.: 1972a, Ann. Phys. (NY) 74, 391

Gilmore, R.: 1972b, Rev. Mex. Fis. 23

Glauber, R.: 1963, Phys. Rev. B 1, 2766

Greiner, M., Mandel, O., Esslinger, T., Hansch, T., and Bloch, I.: 2002, Nature 415, 39

Hagelstein, P. and Chaudhary, I.: 2008, J. Phys. B 41, 135501

Hagelstein, P., McKubre, M., Nagel, D., Chubb, T., and Hekman, R.: 2006, Condensed Matter Nuclear Science, Proceedings of the 11th International Conference on Cold Fusion p. 23

Haldane, F.: 1988, Phys. Rev. Lett. 60, 635

Hollenhorst and N., J.: 1979, Phys. Rev. D 19, 1669

Hong, C. and Mandel, L.: 1985, Phys. Rev. Lett. 54, 323

Husimi, K.: 1940, Proc. Phys. Soc. Japan 22(264)

Imai, H.and Hayash, M.: 2006, Quantum Computation and Information: From Theory to Experiment.(Topics in Applied Physics), Spinger-Verlag

Inozemtsev, V.: 1995, J. Phys. A 28, L445

Inozemtsev, V. and Dorfel, B.: 1993, J. Phys. A 26, L999

Inozemtsev, V. and Inozemtseva, N.: 1991, J. Phys. A 24, L859

Inozemtsev, V. and Kuzemsky, A.: 1991, Phys. Rev. B 43, 1090

Inozemtseva, N. and Inozemtsev, V.: 1997, J. Phys. A 30, L137

Jaksch, D., Briegel, H., Cirac, J., Gardiner, C., and Zoller, P.: 1999, Phys. Rev. Lett. 82, 1975

Jaksch, D., Bruder, C., Cirac, J., Gardiner, C., and Zoller, P.: 1998, Phys. Rev. Lett. 
$\mathbf{8 1}, 3108$

Jaksch, D., Cirac, J., Zoller, P., Rolston, S., Cote, R., and Lukin, M.: 2000, Phys. Rev. Lett. 85, 2208

Javanainen, J. and Gould, P.: 1990, Phys. Rev. A 41, 5088

Jaynes, E. and Cummings, F.: 1963, Proc. IEEE 51, 89

Johnson, T., Urban, E., Henage, T., Isenhower, L., Yavuz, D., Walker, T., and Saffman, M.: 2008, Phys. Rev. Lett. 100, 113003

Joo, J., Lim, Y., Beige, A., and Knight, P.: 2006, Phys. Rev. A 74, 042344

Josse, V., Dantan, A., Bramati, A., and Giacobino, E.: 2004, J. Opt. B 6, S532

Kiffner, M., Evers, J., and Keitel, C.: 2007, Phys. Rev. A 75, 032313

Kitagawa, M. and Ueda, M.: 1993, Phys. Rev. A 47, 5138

Klauder, J. and Sudarshan, E.: 2006, Fundamentals of Quantum Optics, Dover Publications

Klein, A. and Marshalek, E.: 1991, Rev. Mod. Phys. 63, 375

Klimov, A., Negro, J., Farias, R., and Chumakov, S.: 1999, J. Opt. B 1, 562

Kok, P., Munro, W., Nemoto, K., Ralph, T., Dowling, J., and Milburn, G.: 2007, Rev. Mod. Phys. 79, 135

Korbicz, J., Gühne, O., Lewenstein, M., Häffner, H., Roos, C., and Blatt, R.: 2006, Phys. Rev. A 74, 052319

Kuzmich, A., Bigelow, N., and Mandel, L.: 1998, Europhysics Lett. 42, 481

Kuzmich, A., Mandel, L., and Bigelow, N. P.: 2000, Phys. Rev. Lett. 85, 1594

Kuzmich, A., Møler, K., and Polzik, E.: 1997, Phys. Rev. Lett. 79, 4782

Leibfried, D., Blatt, R., Monroe, C., and Wineland, D.: 2003, Rev. Mod. Phys. 75, 281

Leonhardt, U.: 1997, Cambridge Studies in Modern Optics

Leroux, I. D., Schleier-Smith, M. H., and Vuletić, V.: 2010a, Phys. Rev. Lett. 104, 073602

Leroux, I. D., Schleier-Smith, M. H., and Vuletić, V.: 2010b, Phys. Rev. Lett. 104, 250801

Lett, P., Short, R., and Mandel, L.: 1984, Phys. Rev. Lett. 52, 341 
Lukin, M., Fleischhauer, M., Cote, R., Duan, L., Jaksch, D., Cirac, J., and Zoller, P.: 2001, Phys. Rev. Lett. 87, 379011

Lyakhov, A., Braun, D., and Bruder, C.: 2007, Phys. Rev. A 76, 0222321

MacOvei, M., Evers, J., and Keitel, C.: 2005, Phys. Rev. A 71, 033802

Messikh, A., Ficek, Z., and Wahiddin, M.: 2003, Phys. Rev. A 68(064301)

Monroe, C., Meekhof, D., King, B., Itano, W., and Wineland, D.: 1995, Phys. Rev. Lett. 75, 4714

Nielsen, A. and Mølmer, K.: 2008, Phys. Rev. A 77, 063811

Nielsen, M. and Chuang, I.: 2000, Quantum Computation and Quantum Information, Cambridge

Oblak, D.: 2005, Phys. Rev. 71, 043807

Peng, J. and Li, G.: 1998, Introduction to Modern Quantum Optics, World Scientific

Perelomov, A.: 1972, Commun. Math. Phys. 26(222)

Pøulsen, U. and Mølmer, K.: 2001, Phys. Rev. Lett. 87, 123601

Power, E.: 1964, Introductory Quantum Electrodynamics, Longmans

Prakash, H. and Kumar, R.: 2005, J. Opt. B 7, S747

Rabl, P. and Zoller, P.: 2007, Phys. Rev. A 76, 042308

Radcliffe, J.: 1971, J. Phys. A 4(313)

Rangel, R., Carvalho, R., and Zagury, N.: 2005, J. Phys. B 38, 729

Reboiro, M.: 2008, Phys. Scr. 78, 045006

Reboiro, M., Civitarese, O., and Rebon, L.: 2007, Phys. Lett. A 366, 241

Robertson, H. P.: 1929, Phys. Rev. 34, 163

Rojo, A.: 2003, Phys. Rev. A 68, 013807

Romero-Isart, O., Eckert, K., and Sanpera, A.: 2007, Phys. Rev. A 75, 050303

Rubin-Linares, G. and Moya-Cessa, H.: 2005, J. of Mod. Opt. 12, 1751

Saffman, M. and Mølmer, K.: 2008, Phys. Rev. A 78, 012336

Saffman, M. and Mølmer, K.: 2009, Phys. Rev. Lett. 102, 240502

Saffman, M. and Walker, T.: 2005, Phys. Rev. A 72, 0233347

Schanabel, R.: 2008, Nature Phys. 4, 1669

Schleier-Smith, M. H., Leroux, I. D., and Vuletić, V.: 2010a, Phys. Rev. A 81, 
021804

Schleier-Smith, M. H., Leroux, I. D., and Vuletić, V.: 2010b, Phys. Rev. Lett. 104, 073604

Scully, M. and Zubairy, M.: 1997, Quantum Optics, Cambridge Univ. Press

Shaffman, M., Oblak, D., Appel, J., and Polzik, S.: 2009, Phys. Rev. A 79, 023831

Shastry, B.: 1988, Phys. Rev. Lett. 60, 639

Sherson, J., Krauter, H., Olsson, R., Julsgaard, B., Hammerer, K., Cirac, I., and Polzik, E.: 2006, Nature 443, 557

Slusher, R. E., Hollberg, L. W., Yurke, B., Mertz, J. C., and Valley, J. F.: 1985, Phys. Rev. Lett. 55, 2409

Sørensen, A. and Mølmer, K.: 1999, Phys. Rev. Lett. 83, 2274

Takahashi, Y., Honda, K., Tanaka, N., Toyoda, K., Ishikawa, K., and Yabuzaki, T.: 1999, Phys. Rev. A 60, 4974

Takano, T., Fuyama, M., Namiki, R., and Takahashi, Y.: 2009, Phys. Rev. Lett. 102, 033601

Takano, T., Tanaka, S.-I.-R., Namiki, R., and Takahashi, Y.: 2010, Phys. Rev. Lett. 104, 013602

Takei, N., Aoki, T., Koike, S., Yoshino, K., Wakui, K., Yonezawa, H., Hiraoka, T., Mizuno, J., Takeoka, M., Ban, M., and Furusawa, A.: 2005, Phys. Rev. A 72, 1

Takeuchi, M., Ichihara, S., Takano, T., Kumakura, M., Yabuzaki, T., and Takahashi, Y.: 2005, Phys. Rev. Lett. 94, 023003

Tan, H., Xias, H., and Li, G.: 2009, Phys. Rev. A 79, 063805

Tavis, M. and Cummings, F.: 1968, Phys. Rev. 170, 379

Teper, I., Vrijsen, G., Lee, J., and Kasevich, M.: 2008, Phys. Rev. A 78, 051803R

Troinai, F.: 2005, Phys. Rev. Lett. 94, 207208

Van Kampen, H., Sautenkov, V., Eliel, E., and Woerdman, J.: 2000, Opt. Commun. 180,81

Varada, G. and Agarwal, G.: 1992, Phys. Rev. A 45, 6721

Vogel, W. and Blatt, R.: 1992, Phys. Rev. A 45, 3319

Wadkiewicz, K., Knight, P., Buckle, S., and Barnett, S.: 1987, Phys. Rev. A 35, 
2567

Walker, T. and Saffman, M.: 2008, Phys. Rev. A 77, 032723

Walls, D.: 1983, Nature 306, 141

Walls, D.: 1986, Nature 324, 210

Walls, D. and Zoller, P.: 1981, Phys. Rev. Lett. 47, 709

Wang, C.: 2009, Phys. Rev. A 79, 043810

Wang, X.: 2001, J. Opt. B 3, 93

Wang, X. and Sanders, B.: 2003, Phys. Rev. A 68, 033821

Wineland, D.J., B.-J. I. W. H. D.: 1994, Phys. Rev. A 50(1), 67

Yavuz, D.: 2006, Phys. Rev. Lett. 96, 063001

Yonezawa, H., Braunstein, S., and Furusawa, A.: 2007, Phys. Rev. Lett. 99, 110503

Yukalov, V. and Yukalova, E.: 2004, Phys. Rev. A 70, 053828

Zachos, C., Fairlie, D., and Curtright, T.: 2005, Quantum Mechanics in phase space, World Scientific

Zeng, B., Zhou, D., Xu, Z., and You, L.: 2005, Phys. Rev. A 71, 042317

Zhelobenko, D.: 1973, Am. Math. Soc., Providence, R.I. 40

Zhu, Y. and Lin, J.: 1996, Phys. Rev. A 53, 1767 